Florida International University FIU Digital Commons

FIU Electronic Theses and Dissertations

University Graduate School

3-16-2006

\title{
Exotic meson decay widths using lattice quantum chromodynamics
}

Merritt S. Cook

Florida International University

DOI: $10.25148 /$ etd.FI14061502

Follow this and additional works at: https://digitalcommons.fiu.edu/etd

Part of the Physics Commons

\section{Recommended Citation}

Cook, Merritt S., "Exotic meson decay widths using lattice quantum chromodynamics" (2006). FIU Electronic Theses and Dissertations. 2526.

https://digitalcommons.fiu.edu/etd/2526

This work is brought to you for free and open access by the University Graduate School at FIU Digital Commons. It has been accepted for inclusion in FIU Electronic Theses and Dissertations by an authorized administrator of FIU Digital Commons. For more information, please contact dcc@fiu.edu. 


\section{FLORIDA INTERNATIONAL UNIVERSITY \\ Miami, Florida}

\section{EXOTIC MESON DECAY WIDTHS USING}

LATTICE QUANTUM CHROMODYNAMICS

A dissertation submitted in partial fulfillment of the requirements for the degree of DOCTOR OF PHILOSOPHY

in

PHYSICS

by

Merritt S. Cook

2006 
To: Interim Dean Mark Szuchman

College of Arts and Sciences

This dissertation, written by Merritt S. Cook, and entitled Exotic Meson Decay Widths using Lattice Quantum Chromodynamics, having been approved in respect to style and intellectual content, is referred to you for judgment.

We have read this dissertation and recommend that it be approved.

Rajamani Narayanan

Misak Sargsian

Brian Rave

John T. Landrum

H. Rudolf Fiebig, Major Professor

Date of Defense: March 16, 2006

The dissertation of Merritt S. Cook is approved.

Interim Dean Mark Szuchman College of Arts and Sciences

Interim Dean Stephan L. Mintz University Graduate School

Florida International University, 2006 
(C) Copyright 2006 by Merritt S. Cook

All rights reserved. 


\section{ACKNOWLEDGMENTS}

I want to thank all my committee members for their support and guidance during this project. I especially want to thank Professor Fiebig for suggesting this project as a dissertation topic, and for carrying the weight of this project around for a number of years.

I also want to acknowledge financial support from the National Science Foundation under Grant No. PHY - 0300065. This grant allowed me to focus on the research very intensely and get it completed in a reasonable time period. 


\author{
ABSTRACT OF THE DISSERTATION \\ EXOTIC MESON DECAY WIDTHS \\ USING LATTICE QUANTUM CHROMODYNAMICS \\ by \\ Merritt S. Cook \\ Florida International University, 2006 \\ Miami, Florida \\ Professor H. Rudolf Fiebig, Major Professor
}

Most experiments in particle physics are scattering experiments, the analysis of which leads to masses, scattering phases, decay widths and other properties of one or multi-particle systems. Until the advent of Lattice Quantum Chromodynamics (LQCD) it was difficult to compare experimental results on low energy hadronhadron scattering processes to the predictions of QCD, the current theory of strong interactions. The reason being, at low energies the QCD coupling constant becomes large and the perturbation expansion for scattering amplitudes does not converge. To overcome this, one puts the theory onto a lattice, imposes a momentum cutoff, and computes the integral numerically. For particle masses, predictions of LQCD agree with experiment, but the area of decay widths is largely unexplored.

LQCD provides $a b$ initio access to unusual hadrons like exotic mesons that are predicted to contain real gluonic structure. To study decays of these type resonances the energy spectra of a two-particle decay state in a finite volume of dimension $L$ can be related to the associated scattering phase shift $\delta(k)$ at momemtum $k$ through exact formulae derived by Lüscher. 
Because the spectra can be computed using numerical Monte Carlo techniques, the scattering phases can thus be determined using Lüscher's formulae, and the corresponding decay widths can be found by fitting Breit-Wigner functions.

Results of such a decay width calculation for an exotic hybrid $(h)$ meson $\left(J^{P C}=\right.$ $\left.1^{-+}\right)$are presented for the decay channel $h \rightarrow \pi a_{1}$. This calculation employed Lüscher's formulae and an approximation of LQCD called the quenched approximation. Energy spectra for the $h$ and $\pi a_{1}$ systems were extracted using eigenvalues of a correlation matrix, and the corresponding scattering phase shifts were determined for a discrete set of $\pi a_{1}$ momenta. Although the number of phase shift data points was sparse, fits to a Breit-Wigner model were made, resulting in a decay width of about $60 \mathrm{MeV}$. 
1 Introduction 1

1.1 The Standard Model and QCD . . . . . . . . . . . . . . . . 1

1.2 Hadrons, the quark model, and exotic mesons . . . . . . . . . 2

1.3 Experimental situation, JLab program. . . . . . . . . . . . . . 4

1.4 QCD fundamentals. . . . . . . . . . . . . . . 5

1.5 Project goals. . . . . . . . . . . . . . . . 9

2 Basic LQCD 11

2.1 Path integral approach . . . . . . . . . . . . . . 11

3 Operator construction 22

3.1 Quantum numbers and discrete symmetries. . . . . . . . . . 22

3.2 Equal time correlators. . . . . . . . . . . . . . . . . . . . . . . . . . . . . .

3.3 Forming the contractions and propagators . . . . . . . . 30

3.4 Smearing of fields . . . . . . . . . . . . . . 39

4 Simulation and analysis 43

4.1 Goal of this simulation. . . . . . . . . . . . . . . . . 43

4.2 Lattice parameters. . . . . . . . . . . . . . . . . . . . . 43

4.3 Extrapolation to the physical mass region, and setting the physical scale. . . . . . . . . . . . . . . . . . . . . 4 48

4.4 Projecting vs. diagonalizing at all t . . . . . . . . 52

$\begin{array}{lll}5 & \text { Lüscher's method for scattering phase shifts } & 59\end{array}$

5.1 Basic idea behind Lüscher's formulae. . . . . . . . . . . . . . . . 59

5.2 Application of Lüscher's formulae. . . . . . . . . . . . . . . . 63

5.3 The Breit-Wigner function and decay widths. . . . . . . . . 6 65

6 Decay width results 66

6.1 Results for scattering phase shifts and decay widths . . . . . . . 68

6.2 Systematic errors . . . . . . . . . . . . . . . . 75

7 Summary and Conclusion . . . . . . . . . . . . . . 77

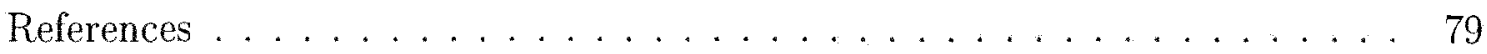

Appendices ......................... 85

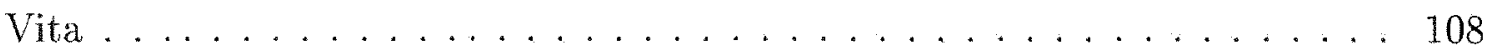




\section{LIST OF TABLES}

TABLE

PAGE

1 Kappa values and pion masses, $12^{3} \times 24$ lattice . . . . . . . . . 46

2 Extrapolated masses of the $\pi, a_{1}, h$ for $L^{3} \times 24 \ldots \ldots \ldots$

3 Energy specta from the $12^{3} \times 24$ lattice . . . . . . . . . . . 58

4 Energy spectra $W_{n}, n=1 \ldots 6$ and phase shifts. . . . . . . . 69

$5 \quad$ Results for decay widths $\Gamma \ldots \ldots \ldots 71$ 
FIGURE

PAGE

$1 \quad$ Plaquette variable $U_{\mu \nu}(x) \ldots \ldots \ldots \ldots \ldots$

2 Depiction of the hybrid exotic operator ........... 24

3 Matrix element diagrams . . . . . . . . . . . . . 38

4 Effective mass of the $a_{1}$ meson . . . . . . . . . 45

5 Effective mass of the exotic hybrid ............ 47

6 Extrapolation, $12^{3} \times 24$ lattice . . . . . . . . . . . 48

$7 \quad$ Extrapolation, $10^{3} \times 24$ lattice . . . . . . . . . . . 49

8 Eigenvalues, projected and diagonalized on every timeslice .... . 55

9 Eigenvalues for the $12^{3} \times 24$ lattice . . . . . . . . . 5 56

10 Energy spectra for the $12^{3}$ and $10^{3} \times 24$ lattice . . . . . . . . 57

11 Generic solutions of a Schrödinger equation . . . . . . . . . . 60

12 Plot of $\delta / \pi q^{2}$ versus $q^{2} \ldots \ldots \ldots \ldots$. . . . . . . . 64

13 Effective masses for the hybrid meson and $a_{1}+\pi \ldots \ldots 7$

14 Resonant scattering phase shifts $\delta_{n} \ldots \ldots \ldots 73$

15 Resonant scattering phase shifts both lattices. . . . . . . . . . 74

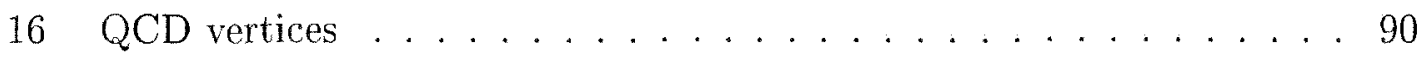

17 Gluon exchange . . . . . . . . . . . . . . . . 91

18 Correlation functions and spectral densities . . . . . . . . . 95 
19 Eigenvalues diagonalized and normalized at $t=5,12^{3} \times 24 \ldots 96$

20 Eigenvalues diagonalized and normalized at $t=5 \ldots . . . . . . .96$

21 Effective masses for all six eigenvalues, $12^{3} \times 24$. . . . . . . . . . 97

22 Effective masses for all six eigenvalues, $10^{3} \times 24$. . . . . . . . . . 98

23 Energy spectrum for the $12^{3} \times 24$ lattice . . . . . . . . . . . . 99

24 Energy spectrum for the $10^{3} \times 24$ lattice . . . . . . . . . . . . 99

25 Energy spectrum for the $8^{3} \times 24$ lattice .............. . . . . . . . . .

26 Energy spectrum for the $12^{3} \times 24$ lattice .............. . . 100

27 Energy spectrum for the $10^{3} \times 24$ lattice . . . . . . . . . . . . 101

28 Energy spectrum for the $8^{3} \times 24$ lattice . . . . . . . . . . . . 101

29 Exotic hybrid correlation function for 2 smearings . . . . . . . 102

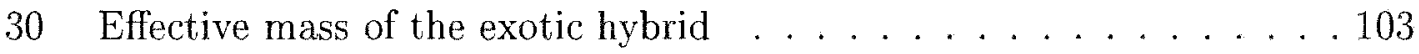

31 Effective mass of the a1 meson, $12^{3} \times 24$ lattice . . . . . . . . 103

32 Effective masses, hybrid and $a_{1}+\pi, 10^{3} \times 24$ lattice........ . 104

33 Effective masses, hybrid and $a_{1}+\pi, 12^{3} \times 24$ lattice . . . . . . 104

34 Effective masses of the a1 meson, $10^{3} \times 24$ lattice ......... . . 105

35 Effective masses of the a1 meson, $12^{3} \times 24$ lattice . . . . . . . . 105

36 Correlation function for the a1 meson on $12^{3} \times 24$ lattice . . . . . . 106

37 Lattice constants for the $12^{3} \times 24$ lattice . . . . . . . . . . . 106

38 Lattice constants for the $10^{3} \times 24$ lattice . . . . . . . . . . 107 


\section{Introduction}

\subsection{The Standard Model and QCD}

The Standard Model of particle interactions [1,2] embodies three of the four fundamental forces in nature - the nuclear strong force, the nuclear weak force, and the electromagnetic force. Within the structure of the Standard Model, the basic constituents of matter are six quarks $u, d, s, c, b, t$ called flavors and six leptons $e, \nu_{e}, \mu, \nu_{\mu}, \tau, \nu_{\tau}$. Forces between these particles are mediated by gauge fields consisting of either gluons (strong force), $W^{ \pm}$and $Z$ bosons (weak force), or photons (electromagnetic force). A gauge theory based on the symmetry group $\mathrm{SU}(3) \times$ $\mathrm{SU}(2) \times \mathrm{U}(1)$, combined with spontaneous symmetry breaking, accounts for all observed interactions. The SU(3) group reflects the symmetry of the strong force where each quark has three color components. SU(3) transformations mix colors and are the basis of Quantum Chromodynamics (QCD). There are eight massless gluons corresponding to the eight generators that make up the Lie algebra of SU(3).

The interaction between quarks and gluons is determined by a strong coupling $\alpha_{s}$. This coupling is energy scale dependent and is known as a running coupling (see Appendix E). Near energy scales of $\approx 1 \mathrm{GeV}$ it's size is $\approx 1$. This strong coupling makes the use of perturbation theory in $\alpha_{s}$ or $\frac{1}{\alpha_{s}}$ impossible, and therefore, the masses of mesons and baryons cannot be calculated perturbatively. The problem is, however, well defined within the path intergral formalism of $\mathrm{QCD}$, although it is intractable by analytical means. To circumvent this difficulty one puts QCD onto a 4-d space-time lattice and computes a functional or path integral by numerical simulation, using the coupling strength as an input parameter. 


\subsection{Hadrons, the quark model, and exotic mesons}

Particles that interact through the nuclear strong force are called hadrons. Examples of hadrons are baryons and mesons. Baryons (e.g.protons, neutrons) contain three quarks or three anti-quarks while mesons (e.g.pions) are composed of quarkantiquark pairs. These three-quark and quark-antiquark combinations can exist in many states of excitation, resulting in the multitude of strongly interacting particles seen in experiments [3].

In fact, this picture is still too simple because the vacuum of this quantum field theory is complicated and thus contributes to the structure of hadrons. Also, QCD may allow the existence of particles made only from gluons, so called glueballs, and particles made from quarks and valence gluons.

The quark model is a classification scheme for hadrons in terms of their valence quarks only [4]. Combinations of valence quarks give rise to sets of quantum numbers used to identify the hadron. Quarks are assigned effective (constituent) masses, and hadrons are constructed from (non-relativistic) wave functions involving color, spin, flavor (isospin), and an orbital component. The most extensive application of this model to obtain the hadron spectrum was done by Isgur and collaborators $[5,6]$.

Quarks have spin $\frac{1}{2}$ and by convention have positive parity $P=+1$. Anti-quarks have the opposite parity. Thus the parity of mesons is $P=(-1)^{l+1}$ where $l$ is the orbital angular momentum quantum number [4]. A phase factor of $(-1)^{l}$ comes from inverting the relative position vector between the quarks with an additional factor of $(-1)$ due to the opposite intrinsic parity of a quark and anti-quark. The total meson spin $J$ is in the range $|l-s|<J<|l+s|$ where $s$ can be 0 (anti-parallel quark spins) or 1 (parallel quark spins). 
The charge conjugation operator converts a particle to it's anti-particle. If a meson state is a charge conjugation eigenstate, then C-parity is determined by $C=$ $(-1)^{l+s}[4]$. This includes phase factors of $(-1)^{l+1}$ for inverting the relative position vector during the exchange, a factor of $(-1)^{s}$ because the spin polarization vectors of quarks and anti-quarks have opposite sign, and a factor of $(-1)$ for exchanging a quark with it's anti-quark. Mesons can then be classified in terms of spin-paritycharge $\left(J^{P C}\right)$ multiplets. States with parity $P=(-1)^{J}$ are called natural spin-parity states and can have $s=1$ with $C P=+1$ or $s=0$ with $C P=-1$. Mesons with natural spin-parity and $s=1$ cannot have $C P=-1$. Such states, although allowed within QCD, are not accessible within the quark model.

Mesons with quantum numbers that cannot be obtained with the (valence) quark model are called exotic. Hybrid mesons are quark-antiquark pairs having valence gluons as a structural component. When their quantum numbers are not accessible with quark models they are called hybrid exotics. Examples of these exotics are the $J^{P C}=0^{+-}, 1^{-+}, 2^{+-}$mesons. All of these states are unstable and reveal themselves in experiments only as resonances at best. A resonance has a mass and a width that is related to the decay constant of the particle. Because these mesons contain real gluons, as opposed to the virtual gluons responsible for the nuclear strong force, the verification of their existence is a signature test for QCD.

In order to describe valence gluon degrees of freedom, which are not contained as such in the quark model $[5,6]$, Isgur and Paton constructed a flux tube model $[7,8]$. Predictions for the smallest hybrid meson masses fall in the region just below $2 \mathrm{GeV}$. Decay modes and partial widths can also be studied. 
For selected hybrid meson states it is found that the decay products preferentially involve an excited meson [9], which complicates their detection. The lightest hybrid meson is predicted around $1.9 \mathrm{GeV}$ with $J^{P C}=1^{-+}[10,11]$. According to Close and Page [12], in the flux tube model the channels $a_{1} \pi, b_{1} \pi, f_{1} \pi$ and $\rho \pi$ have partial decay widths of $100,170,60$ and $5-20 \mathrm{MeV}$, respectively.

\subsection{Experimental situation, JLab program.}

Efforts to determine properties of these hybrid exotic states are unsettled from both experimental and theoretical viewpoints [13]. The experimental efforts date back over a decade, and currently, considerable resources are being devoted to their future study. The Jefferson Lab GlueX experimental program is designed to investigate exotic states [14]. Hybrid meson studies are also part of the COMPASS experiment at CERN [15] and the CLEO-c program [16] at Cornell.

Several observations of the $J^{P C}=1^{-+}$exotic meson have been previously reported:

- by Brookhaven National Lab. (in $\pi n$ interactions at $18 \mathrm{GeV}$ ) at mass $1370 \pm$ $16 \mathrm{MeV}$ with decay width $\Gamma=385 \pm 40 \mathrm{Mev}$, decaying into $\pi \eta, \pi \eta^{\prime}$ and $\pi f_{1}$ channels $[17,18]$.

- by Cristal Barrel Collaboration at LEAR (in $\bar{p} n$ interactions) at mass $1400 \pm 20$ $\mathrm{MeV}$ and $\Gamma=310 \pm 50 \mathrm{Mev}$, decaying into $\pi \eta[19]$. 
- by the SLAC Hybrid Facility (in $\gamma p$ interactions) at mass $1775 \mathrm{MeV}$ and $\Gamma=100$ to $200 \mathrm{Mev}$, decaying into $\pi \rho$ and $\pi f_{2}[20]$.

- by E852 at BNL at mass $1593 \pm 8 \mathrm{MeV}$ and $\Gamma=168 \pm 20 \mathrm{Mev}$, decaying into $\pi \rho[21,18]$.

- by E852 at BNL at mass $1597 \pm 10 \mathrm{MeV}$ and $\Gamma=340 \pm 40 \mathrm{Mev}$, decaying into $\pi \eta^{\prime}[22]$.

\subsection{QCD fundamentals.}

Quantum Chromodynamics (QCD) is a quantized gauge field theory of strongly interacting matter based on the SU(3) symmetry of the Standard Model. The elementary degrees of freedom of QCD are called quarks and gluons. The gluons are the gauge fields of the theory and are responsible for mediating the interaction analogous to the quanta (photons) of an electromagnetic field.

Quarks are the matter fields of QCD and exist as six flavors (up, down, strange, charmed, bottom, and top) and they are assigned a baryon number that is conserved $\left(\frac{1}{3}\right.$ for quarks $(q),-\frac{1}{3}$ for anti-quarks $(\bar{q})$ ). In Nature most of the different quarks have vastly different masses [3]. Quarks also carry a quantum number associated with the SU(3) gauge symmetry group, known as color. In the fundamental representation of $\mathrm{SU}(3)$ the color quantum number assumes three values. An arbitrary assignment is three primary colors (red(r), green(g), or blue(b)) for quarks (see Appendix D). The complex conjugate representation describing anti-quarks also has dimension three $(\operatorname{anti-red}(\bar{r}), \operatorname{anti-green}(\bar{g})$, or anti-blue $(\bar{b}))$. 
The colors of quarks, or anti-quarks, cannot be distinguished experimentally. Thus one requires that arbitary transformations of colors, described by elements of SU(3), may be performed at any space-time point without having observable consequences. This symmetry is known as local gauge invariance. It inescapably entails the presence of a gauge field, called the gluon field in QCD. Gluons transform according to the adjoint representation of $\mathrm{SU}(3)$, meaning that there are eight $\left(3^{2}-\right.$ $1=8$ ) colors of gluons. One may think of these as a subset of color-anticolor combinations; $r \bar{b}, r \bar{g} \ldots$ etc, see Appendix D.

Local gauge invariance is a severe constraint on the structure of a theory. It determines completely the type of elementary interaction vertices. In the case of QCD, these are shown in Fig. 16, Appendix D.

The only free parameters that emerge are the strong coupling $\alpha_{s}$, and the quark masses. In contrast to quantum electrodynamics (QED), where a pure photon interaction vertex does not exist, the gluons of QCD do interact.

The physical consequences of this feature are profound. Applying renormalization group techniques to QCD, it was shown by Gross, Wilczek and Politzer in 1973 $[23,24]$ that the scale dependence of the strong coupling $\alpha_{s}$ is such that it goes to zero at small distances (or at large momenta or energy, see Appendix E). This property, known as asymptotic freedom, allows use of perturbation theory for high energy processes, but for low energy processes this is not possible.

It was shown by Wilson in 1974 [25] that objects with color separated by a spatial distance $r$ experience an attractive potential $V(r)$ that rises linearly with $r$ as $r \rightarrow \infty$ (small momenta). 
This feature, known as confinement, explains the observation that isolated colored objects are not seen experimentally. In fact only colorless (white) objects are seen in nature as asymptotic states. The simplest ones are $\bar{q} q$ pairs or mesons with color $\bar{r} r=$ white, etc., and $q q q, \bar{q} \bar{q} \bar{q}$, or baryons with color $r g b=$ white, etc. Four and five quarks states are also allowed, but at this time, it is not clear if these states have a compact structure or resemble hadronic molecules. Furthermore, self-interaction of the gluon field may give rise to a unique type of hadron only made from glue, so-called glueballs, and to hybrid hadrons as mentioned above, which are the subject of this thesis.

The physics in the mass range relevant for hadrons, say below $\approx 2 \mathrm{GeV}$, is dominated by confinement. The only known practical ab initio access to (strong) hadronic physics is the lattice regulated formulation of QCD combined with numerical simulation.

In a seminal work in 1974 Kenneth Wilson [25] introduced a space-time lattice formulation using a path integral technique for field quantization. As mentioned above, he showed that for sufficiently strong coupling QCD exhibits the property of confinement. In Euclidean space-time the theory resembles a discrete statistical mechanics system. This feature was employed by Creutz [26] who showed in 1980 how to calculate hadron masses by numerical simulation, originally using an SU(2) gauge group. Hamber, Parisi, and Weingarten in 1981 [27, 28] extended this technique to the calculation of hadron masses in $\mathrm{SU}(3)$, as described in Section 2. 
Theoretical work on hybrid mesons using LQCD has so far concentrated mainly on their ground state masses. It can be roughly classified in terms of heavy quark systems using static quarks with valence glue treated in the Born-Oppenheimer approximation [29, 30, 31], using non-relativistic LQCD [32, 33, 34], and studies using actions with both quenched and unquenched quark dynamics $[35,36,37,38$, $39,40,41,42,43]$.

Until now, there has been no $a b$ initio lattice QCD based prediction of hybrid decay widths using light quarks. Hybrid decay widths have only been studied on the lattice in the heavy quark limit [44].

Resonances, being a dynamic phenomenon, do not relate to eigenstates of a Hamiltonian in a simple way. They are superpositions of partial waves with each partial wave characterized by it's own angular momentum $(l)$ and phase shift.

Calculating properties of resonances using Euclidean lattice QCD simulations is difficult for a number of reasons. First, as shown by Maiani and Testa [45], in Euclidean space-time the scattering amplitudes are real, thus preventing extraction of phase shifts and resonant structures. Second, on a finite volume lattice the spectrum is discrete and all states are bound. Third, the smallest total energy of a two-hadron state may be larger than the energy of the original hadron thus preventing decay. Aspects of these points have been discussed by Michael [46, 47], DeGrand [48], Lüscher [49], and by Lellouch and Lüscher [50].

A solution to these difficulties was first proposed for lattice QCD by Lüscher $[49,51]$. He showed how the discrete two-particle energy spectrum in a finite periodic box is related to continuum elastic scattering amplitudes. A decay width can then be extracted by fitting a Breit-Wigner model, if applicable. 
Feasability of this method has been demonstrated for the $O(3)$ non-linear sigma model in $1+1$ dimensions [52], the $O(4)$ non-linear sigma model in $3+1$ dimensions [53, 54], meson-meson scattering in 2+1 dimensions using QED [55], and resonance scattering of two coupled Ising systems $[56,57]$. Scattering lengths have also been studied, for example see $[58,59,60]$, by looking at the lattice volume dependence of low lying two-particle energy levels [61,62], and hybrid decays in the heavy quark limit were investigated in [44].

\subsection{Project goals.}

In this project we apply lattice quantum chromodynamics (LQCD) to calculate the decay width of a $J^{P C}=1^{-+}$hybrid exotic meson (say $h$ ) using Lüscher's method. In a partial wave decomposition of the $1^{-+}$decay, this exotic meson couples to the $\pi+a_{1}$ channel in which the two mesons emerge in a relative s-wave $(l=0)$. This turns out to be crucial for the feasibility of the lattice simulation because the mass associated with the relative kinetic energy in the two-body decay channel is zero, and therefore, the lattice masses of the exotic state and the two-meson state are expected to be close. For that reason, this project focused on the $1^{-+}$decay.

Thus the primary goal of the LQCD simulation is to obtain the mass spectrum with as many excited states as can be determined of the coupled $h, \pi+a_{1}$ system. The spectra will be used to calculate scattering phase shifts at a discrete set of momenta by applying Lüscher's method. Fitting a Breit-Wigner function, if successful, should then yield an estimate for the decay width. 
The thesis is organized as follows: Section 2 introduces the path integral formulation of LQCD in terms of gluon and fermion field actions, the fermion matrix, and quantum operators. Section 2 also describes the use of effective mass functions and correlation functions to calculate expectation values. Section 3 describes in more detail the constuction of operators having the correct quantum numbers, and how these operators are used to compute propagators. Section 4 includes the actual lattice parameters used in this simulation along with a discussion of setting the physical scale and the use of a correlation matrix. Section 5 outlines the basic theory behind Lüscher's method and shows how Lüscher's formulae are used to calculate scattering phase shifts. In Section 6, results are presented for the effective mass functions, scattering phase shifts, and decay widths. Section 7 contains the conclusion. 


\section{Basic LQCD}

\subsection{Path integral approach}

The conventional formulation of a quantum field theory, that is well defined in the non-perturbative regime, is based on a path integral involving a classical action in Euclidean space-time. Euclidean time $t_{E}$ is related to physical or Minkowski time $t_{M}$ through a Wick rotation $t_{E}=-i t_{M}$ where $t_{E}$ is treated as real. A Wick rotation rendors path integrals convergent while leaving dynamic quantities (mass spectra, etc.) unchanged.

The coordinates in 4-d Euclidean space-time are,

$$
x_{1}=x \quad x_{2}=y \quad x_{3}=z \quad x_{4}=c t_{E}
$$

where the metric is $\left(\delta_{\mu \nu}\right)=\operatorname{diag}(1,1,1,1)$ with $\mu, \nu=1,2,3,4$.

The QCD action is the sum of a gauge field and a fermion or quark field action,

$$
S_{Q C D}=S_{\text {gaug }}+S_{\text {ferm }}
$$

The gauge fields or gluons are described by a 4-vector field $A_{\mu}(x), \mu=1,2,3,4$ with an additional color index $a=1 \ldots 8$,

$$
A_{\mu}(x)=A_{\mu}^{a}(x) \lambda_{a}
$$

where the $\lambda_{a}$ are the set of eight Gell-Mann matrices (Appendix B) that generate the Lie-algebra of $\mathrm{SU}(3)$,

$$
\left[\lambda_{a}, \lambda_{b}\right]=2 i f_{a b c} \lambda_{c}
$$

and the $f_{a b c}$ are the corresponding structure constants. 
An important building block of a gauge field theory is the covariant derivative,

$$
D_{\mu}(x)=\partial_{\mu}-i g A_{\mu}(x)
$$

involving a parameter $g$. It's importance derives from the behavior of the actions under local infinitesimal transformations of the form,

$$
A_{\mu}^{a}(x) \rightarrow A_{\mu}^{a}(x)+\frac{1}{g} \partial_{\mu} \omega^{a}(x)+f_{a b c} \omega^{b}(x) A_{\mu}^{c}(x),
$$

with arbitrary real functions $\omega^{a}(x)$. In matrix notation, $A_{\mu}$ transforms as,

$$
A_{\mu} \rightarrow A_{\mu}^{\prime}=\Omega(x)\left(A_{\mu}+\frac{i}{g} \partial_{\mu}\right) \Omega^{\dagger}(x)
$$

with

$$
\Omega(x)=e^{i \lambda_{a} \omega^{a}(x)} \in \mathrm{SU}(3)
$$

Then we have

$$
D_{\mu}(x) \rightarrow D_{\mu}^{\prime}(x)=\Omega(x) D_{\mu}(x) \Omega^{\dagger}(x)
$$

where

$$
D_{\mu}^{\prime}(x)=\partial_{\mu}^{\prime}+i g A_{\mu}^{\prime}(x)
$$

The gluon field strength tensor, defined as

$$
F_{\mu \nu}(x)=\frac{1}{i g}\left[D_{\mu}, D_{\nu}\right]=\partial_{\mu} A_{\nu}(x)-\partial_{\nu} A_{\mu}(x)+i g\left[A_{\mu}(x), A_{\nu}(x)\right],
$$

transforms like the covariant derivative

$$
F_{\mu \nu}(x) \rightarrow F_{\mu \nu}^{\prime}(x)=\Omega(x) F_{\mu \nu}(x) \Omega^{\dagger}(x)
$$

under a gauge transformation. The gauge field action

$$
S_{g a u g}=-\frac{1}{4} \int d^{4} x \operatorname{Tr}\left(F_{\mu \nu} F^{\mu \nu}\right)
$$


where the trace $(\mathrm{Tr})$ refers to color indices, is then invariant with respect to arbitrary local gauge transformations. A theory with a structure like this based on a nonAbelian group, here SU(3), is known as a Yang-Mills theory. Its Abelian version, based on the group U(1), describes Maxwell's theory of electromagnetism.

The fermion, or quark, part of the action is

$$
S_{f e r m}=\int d^{4} x \bar{\Psi}_{f}(x)\left(\gamma_{\mu} D_{\mu}-m_{f}\right) \Psi_{f}(x)
$$

where $\bar{\Psi}_{f}, \Psi_{f}$ is the Dirac field for a quark with flavor $f=u, d, s, \ldots$ and mass $m_{f}$. The Dirac matrices $\gamma_{\mu}$ used for Euclidean space-time are given in Appendix C. The covariant derivative $D_{\mu}$ in (2.14) insures that a local gauge transformation

$$
\Psi_{\mu}(x) \rightarrow \Psi_{\mu}^{\prime}(x)=\Omega(x) \Psi_{\mu}(x)
$$

and

$$
\bar{\Psi}_{\mu}(x) \rightarrow \bar{\Psi}_{\mu}^{\prime}(x)=\bar{\Psi}_{\mu}(x) \Omega^{\dagger}(x)
$$

leaves the fermion action invariant.

The quantization of the theory proceeds through considering the generating functional

$$
Z[J, \chi, \bar{\chi}]=\int[d A][d \Psi][d \bar{\Psi}] e^{-S_{Q C D}+A_{\mu} J_{\mu}+\bar{\Psi} \chi+\bar{\chi} \Psi}
$$

defined through path integrals with appropriate, $\mathrm{SU}(3)$ invariant, measures. The fields $J, \chi, \bar{\chi}$ are sources in the sense that certain functional derivatives of $Z$, taken at vanishing sources, give all propagator functions of the theory. While $A$ and $J$ are $\mathbb{C}$-valued fields, the fermion fields $\Psi, \bar{\Psi}$ and $\chi, \bar{\chi}$ are Grassmann valued, i.e. anticommuting $\mathbb{C}$-numbers [63]. 
As with any quantum field theory in continuous space-time it has to be regulated in order to deal with divergences. In the low energy regime relevant for hadron physics, in terms of a non-perturbative approach, this is done by introducing a discrete space-time lattice. In 1974 Wilson [25] proposed such a discretization in a way that manifestly preserves $\mathrm{SU}(3)$ gauge invariance.

The gauge field is described by so-called link variables $U_{\mu}(x) \in \mathrm{SU}(3)$ connecting neighbor sites $x$ and $x+\hat{\mu}$, where $\hat{\mu}$ is the vector having direction $\mu$ and a length of the lattice constant $a_{\mu}$ in this direction. The smallest closed paths on the lattice are elementary rectangles or plaquettes. Parallel transport around a plaquette with corner $x$ in the $\mu-\nu$ plane $(\mu \neq \nu)$ is provided by the product of four link variables (see Fig. 1),

$$
U_{\mu \nu}(x)=U_{\mu}(x) U_{\nu}(x+\hat{\mu}) U_{\mu}^{\dagger}(x+\hat{\nu}) U_{\nu}^{\dagger}(x)
$$

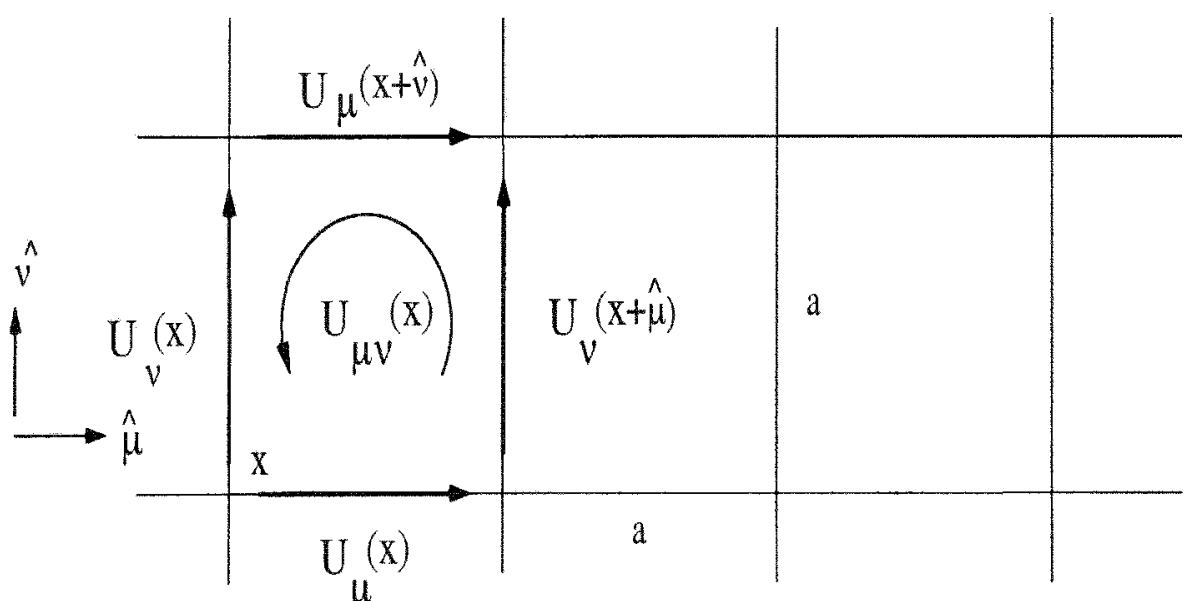

Figure 1: Illustration of the plaquette variable $U_{\mu \nu}(x)$ in the $\mu-\nu$ plane. Here $a$ denotes lattice constants. 
The Wilson action for the pure gauge or gluon field is [64]

$$
S_{g}[U]=\sum_{x} \sum_{1 \leq \mu<\nu \leq 4} \beta_{\mu \nu}\left(1-\frac{1}{3} \operatorname{Re} \operatorname{Tr}\left(U_{\mu \nu}(x)\right)\right) .
$$

where $\operatorname{Tr}$ denotes the trace over $\mathrm{SU}(3)$ color,

$$
\beta_{\mu \nu}=\beta \frac{a_{1} a_{2} a_{3} a_{4}}{\left(a_{\mu} a_{\nu}\right)^{2}}
$$

and $\beta$ is a parameter. This action is based on a finite-volume, discrete space-time lattice with sites,

$$
x=\left(n_{1} a_{1}, n_{2} a_{2}, n_{3} a_{3}, n_{4} a_{4}\right),
$$

where $n_{\mu} \in \mathbb{N}, 1 \leq n_{\mu} \leq N_{\mu}$, for $\mu=1,2,3,4$, and $a_{s, t}$ are lattice constants with dimensions of length. If the spatial and temporal lattice constants, $a_{s}=a_{1}=a_{2}=$ $a_{3}$ and $a_{t}=a_{4}$ respectively are different then we call the lattice anisotropic with aspect ratio $\xi=a_{s} / a_{t}$.

According to Schwinger, a link variable can be related to the continuum gauge field $A_{\mu}^{a}(x)$ via

$$
U_{\mu}(x)=\mathcal{P} \exp \left(i \int_{0}^{1} d s A_{\mu}^{a}(x+s \hat{\mu}) \frac{\lambda_{a}}{2}\right)
$$

where $\mathcal{P}$ denotes path ordering. In the classical continuum limit, $a \rightarrow 0$, with $a_{s}=a$ and $a_{t}=a / \xi$, one recovers $S_{g a u g}$ (see eq. 2.19), provided that

$$
\beta=\frac{6}{g^{2}}
$$

The naive lattice discretization of the Dirac action $S_{q}$, eq. (2.14), has the undesirable property of describing $2^{4}=16$ mass-degenerate species of fermions (one for each corner of the 4-d Brillouin zone) instead of just one (often called the doubling 
problem). There are two popular remedies to this lattice artifact. In the KogutSusskind or staggered scheme [65], one of the 16 fermion field degrees of freedom is isolated through spin-diagonalization (this technique is not used in this work). In the Wilson fermion scheme [66], a second derivative term is added to the naively discretized Dirac action,

$$
\begin{aligned}
S_{q} & =\sum_{x} \sum_{\mu=1}^{4} \bar{\psi}_{f}(x) \gamma_{\mu} \frac{1}{2 a_{\mu}}\left(\psi_{f}(x+\hat{\mu})-\psi_{f}(x-\hat{\mu})\right)+\sum_{x} m_{q} \bar{\psi}_{f}(x) \psi_{f}(x) \\
& -\sum_{x} \sum_{\mu=1}^{4} \frac{1}{2 a_{\mu}} \bar{\psi}_{f}(x)\left(\psi_{f}(x+\hat{\mu})+\psi_{f}(x-\hat{\mu})-2 \psi_{f}(x)\right) .
\end{aligned}
$$

This has the effect that in the classical continuum limit, $a \rightarrow 0,15$ of the 16 fermion species acquire masses with $m \rightarrow \infty$, thus decoupling them from the dynamics of the system and solving the fermion doubling problem.

The fermion fields $\psi, \bar{\psi}$ enter LQCD as integration variables in the path integral. It is customary to rescale these, arriving at

$$
S_{q}=\sum_{x, y} \bar{\psi}_{f}(x) Q(x, y) \psi_{f}(y)
$$

with the quark or fermion matrix defined as

$$
Q(x, y)=\mathbf{1} \delta_{x, y}-\kappa \sum_{\mu} \eta_{\mu}\left(\left(1-\gamma_{\mu}\right) U_{\mu}(x) \delta_{x+\hat{\mu}, y}+\left(1+\gamma_{\mu}\right) U_{\mu}^{\dagger}(y) \delta_{x, y+\hat{\mu}}\right)
$$

Here 1 denotes the identity matrix in color-Dirac space. The hopping parameter $\kappa$ in eq. (2.26) is related to the bare quark mass $m_{q}$ in eq. (2.24) by

$$
\kappa=\frac{\sum_{\lambda} \frac{1}{a_{\lambda}}}{8\left(m_{q}+\sum_{\lambda} \frac{1}{a_{\lambda}}\right)}
$$

and

$$
\eta_{\mu}=\frac{4}{a_{\mu} \sum_{\lambda} \frac{1}{a_{\lambda}}}
$$


Quantization of the lattice theory proceeds through a path integral in the standard way. For example, repeated source derivatives of a generating functional, like eq. (2.17), at vanishing sources gives the expectation value of a general function $\mathcal{O}$ of the lattice field variables,

$$
\mathcal{O}[U, \psi, \bar{\psi}]=\psi\left(y_{1}\right) \bar{\psi}\left(x_{1}\right) \psi\left(y_{2}\right) \bar{\psi}\left(x_{2}\right) \ldots \psi\left(y_{n}\right) \bar{\psi}\left(x_{n}\right) A[U]
$$

where $A[U]$ is the gauge field dependent part of the operator, as [64]

$$
<\mathcal{O}>=\mathcal{Z}_{0}^{-1} \int[d U][d \bar{\psi}][d \psi] e^{-S_{g}[U]-S_{q}[U, \psi, \bar{\psi}]} \mathcal{O}[U, \psi, \bar{\psi}]
$$

where,

$$
\left.\mathcal{Z}_{0}=\int[d U] d \psi\right][d \bar{\psi}] e^{-S_{g}[U]-S_{q}[U, \psi, \bar{\psi}]}
$$

The integrals over the Grassmann fields $\psi, \bar{\psi}$ are Gaussian. They can be performed analytically. For example, one obtains,

$$
\mathcal{Z}_{0}=\int[d U] \operatorname{det} Q[U] e^{-S_{g}[U]}
$$

Another example is the two-point function or quark propagator,

$$
<\psi_{a \mu}(x) \bar{\psi}_{b \nu}(y)>=\mathcal{Z}_{0}^{-1} \int[d U] \operatorname{det} Q[U] e^{-S_{g}[U]} Q_{a \mu, b \nu}^{-1}(x, y)
$$

where $a, b$ are color and $\mu, \nu$ are Dirac indices.

The inverse $Q^{-1}[U]$ of $Q[U]$ is understood as a matrix in $a, \mu, x$-space having size $3 \cdot 4 \cdot N_{1} N_{2} N_{3} N_{4}$. Computation of (columns of) $Q^{-1}[U]$ is one of the major numerical tasks of LQCD simulations. The determinant $\operatorname{det} Q[U]$ still requires an order of magnitude larger computational power. Approximating it with one, $\operatorname{det} Q[U] \cong 1$, is known as the quenched approximation. In physics terms, this means neglecting effects of sea quarks, or closed quarks loops, in the language of Feynman diagrams. 
We wish to study the properties of (color singlet) hadrons. The corresponding operators are combinations of quark-antiquark (meson) or three-quark (baryon) operators, or their products for multi-hadron systems. Let $\mathcal{O}$ be one such operator $(\mathcal{O} \sim$ $\bar{\psi} \psi, \sim \psi \psi \psi$, etc.) Then the corresponding propagator is, in Minkowski space-time,

$$
\begin{aligned}
G\left(t, t_{0}\right) & \left.=<0\left|T \mathcal{O}(t) \mathcal{O}^{\dagger}\left(t_{0}\right)\right| 0\right\rangle \\
& =-: \mathcal{O}(t) \mathcal{O}^{\dagger}\left(t_{0}\right):+T \mathcal{O}(t) \mathcal{O}^{\dagger}\left(t_{0}\right)
\end{aligned}
$$

where : denotes normal ordering, $T$ denotes time-ordering, and $\mid 0>$ is the vacuum state with energy $E_{0}=0$ by convention. A Wick rotation to Euclidean space-time, $t \rightarrow-i t$, transforms the propagator into a time correlation function, $G\left(t, t_{0}\right) \rightarrow$ $\mathcal{C}\left(t, t_{0}\right)$, where

$$
\mathcal{C}\left(t, t_{0}\right)=<\mathcal{O}(t) \mathcal{O}^{\dagger}\left(t_{0}\right)>-<\mathcal{O}(t)><\mathcal{O}^{\dagger}\left(t_{0}\right)>
$$

which is similar to the variance of a stochastic variable. A formal treatment of this relationship involves the transfer matrix formalism, e.g. see [64].

Upon inserting a complete set of $|n\rangle$ eigenstates of the Hamiltonian into eq. (2.34) and performing a Wick rotation one finds that,

$$
\mathcal{C}\left(t, t_{0}\right)=\sum_{n>0} A_{n} e^{-E_{n}\left(t-t_{0}\right)}
$$

where $A_{n}=|<0| \mathcal{O}\left(t_{0}\right)|n>|^{2}$ with $\mid 0>$ being the vacuum state $\left(E_{0}=0\right)$ and $E_{n}$ the energy of $\mid n>$. In the limit of large Euclidean time $t$ the higher energy states decay faster than the ground state, leading to

$$
C\left(t, t_{0}\right) \rightarrow A_{1} e^{-E_{1}\left(t-t_{0}\right)}, \quad \text { for large } t
$$


The so-called effective mass function,

$$
m_{\mathrm{eff}}\left(t, t_{0}\right)=-\frac{\partial}{\partial t} \ln \left[C\left(t, t_{0}\right)\right]
$$

thus developes a plateau,

$$
m_{\mathrm{eff}}\left(t, t_{0}\right) \rightarrow E_{1} \text { for large } t
$$

This is the basis for energy or mass calculations on a lattice.

In practice it is often necessary to employ several operators $\mathcal{O}_{k}(t), k=1 \ldots K$, and thus form a time correlation matrix. Also, to extract the energy spectrum, alternative methods such as variational or maximum entropy techniques can be used (see Appendix G).

To illustrate the calculation of a correlation function consider, as a very simple example, the pseudo-scalar meson operator

$$
\mathcal{O}_{\pi^{+}}(t)=\sum_{\vec{x}} \bar{d}_{a}(\vec{x} t) \gamma_{5} u_{a}(\vec{x} t)
$$

where the sum over colors, $a=1 \ldots 3$, is implied. The sum over all spatial lattice sites $\vec{x}$ makes a translationally invariant operator $\mathcal{O}_{\pi^{+}}(t)$ and it is therefore a zeromomentum operator. The corresponding (Euclidean) correlation function,

$$
\mathcal{C}\left(t, t_{0}\right)=<\mathcal{O}_{\pi^{+}}(t) \mathcal{O}_{\pi^{+}}^{\dagger}\left(t_{0}\right)>-<\mathcal{O}_{\pi^{+}}(t)><\mathcal{O}_{\pi^{+}}^{\dagger}\left(t_{0}\right)>
$$

contains expectation values of operators as defined in eqs. (2.29) and (2.30). By integrating out the fermion fields, $\psi=u$.. etc., in the path integral those can be expressed as elements of the inverse fermion matrix $Q^{-1}$, cf. (2.33). Equivalently, Wick's theorem may be applied to reduce the expectation values in eq. (2.41). For 
example,

$$
\begin{aligned}
<\mathcal{O}_{\pi^{+}}(t) \mathcal{O}_{\pi^{+}}^{\dagger}\left(t_{0}\right)> & =\sum_{\vec{x}} \sum_{\vec{y}}<\bar{d}_{a}(\vec{x} t) \gamma_{5} u_{a}(\vec{x} t) \cdot \bar{u}_{b}\left(\vec{y} t_{0}\right) \gamma_{5} d_{b}\left(\vec{y} t_{0}\right)> \\
& =\sum_{\vec{x}} \sum_{\vec{y}}<-Q_{a \mu, b \nu}^{-1}\left(\vec{x} t, \vec{y} t_{0}\right) \gamma_{5, \nu \nu^{\prime}} Q_{b \nu^{\prime}, a \mu^{\prime}}^{-1}\left(\vec{y} t_{0}, \vec{x} t\right) \gamma_{5, \mu^{\prime} \mu}>
\end{aligned}
$$

where the Dirac indices, $\mu \ldots$ etc., are shown explicitly. For the Wilson fermion action, $Q$ has the property

$$
\gamma_{5} Q(x, y) \gamma_{5}=Q^{\dagger}(y, x)
$$

Hermitean conjugation is understood to affect color and spin indices. Equation (2.43) also applies to $Q^{-1}$. Thus eq. (2.42) becomes

$$
<\mathcal{O}_{\pi^{+}}(t) \mathcal{O}_{\pi^{+}}^{\dagger}\left(t_{0}\right)>=\sum_{\vec{x}} \sum_{\vec{y}}<-\operatorname{Tr} Q^{-1}\left(\vec{x} t, \vec{y} t_{0}\right) Q^{-1^{\dagger}}\left(\vec{x} t, \vec{y} t_{0}\right)>
$$

Finally, translational invariance of the expectation values may be used. Adding $\vec{x}_{0}-\vec{y}$ to every spatial index in $\langle\ldots\rangle$ gives,

$$
\begin{aligned}
<\mathcal{O}_{\pi^{+}}(t) \mathcal{O}_{\pi^{+}}^{\dagger}\left(t_{0}\right)>= & \sum_{\vec{x}} \sum_{\vec{y}}<-\operatorname{Tr} Q^{-1}\left(\vec{x}+\vec{x}_{0}-\vec{y} t, \vec{y}+\vec{x}_{0}-\vec{y} t_{0}\right) \\
& Q^{-1^{\dagger}}\left(\vec{x}+\vec{x}_{0}-\vec{y} t, \vec{y}+\vec{x}_{0}-\vec{y} t_{0}\right)>
\end{aligned}
$$

and then changing the summation variable $\vec{x} \rightarrow \vec{x}-\overrightarrow{x_{0}}+\vec{y}$ gives,

$$
<\mathcal{O}_{\pi^{+}}(t) \mathcal{O}_{\pi^{+}}^{\dagger}\left(t_{0}\right)>=V \sum_{\vec{x}}<-\operatorname{Tr} Q^{-1}\left(\vec{x} t, \overrightarrow{x_{0}} t_{0}\right) Q^{-1^{\dagger}}\left(\vec{x} t, \overrightarrow{x_{0}} t_{0}\right)>
$$

where $V=N_{1} N_{2} N_{3}$ is the spatial volume. Note carefully that $Q^{-1}\left(x, x_{0}\right)$ is needed only for a fixed but arbitrary source lattice site $x_{0}=\left(\vec{x}_{0} t_{0}\right)$. 
The above example highlights the essential workplan of this project. For the exotic meson, in addition to quark fields, gluon fields are needed to construct the operators. This is discussed in Section 3 . 


\section{Operator construction}

\subsection{Quantum numbers and discrete symmetries.}

Simulation of the decay $h \rightarrow \pi+a_{1}$, based on Lüscher's method, requires the construction of quantum operators that create the $1^{-+}$exotic meson $(h)$ ground state and the $\pi a_{1}$ two meson states. In order for these operators to produce a "good signal" in the numerical simulation, it is desireable that they have compatible quantum numbers, meaning they transform as the basis of an irreducible representation (irrep) of the relevant symmetry group.

Transformation properties of quantum fields are used to determine conserved quantities. If a quantum field is invariant under certain continuous transformations, then it will possess a Noether current and there is a corresponding conservation law (quantum number). The field or a function of fields is then said to possess symmetry under the transformation. Both continuous and discrete symmetries exist.

For this lattice project we use zero-momentun operators, like eq. (2.40), discussed in Section 2. However, continuous translations are replaced with a discrete group of translations by multiples of the lattice constants. Likewise the continuous rotation group $O(3)$ is replaced with the discrete group of transformations of a 3-dimensional lattice. We will not encounter this complication because only local operators (defined on one site) are used.

Of concern here are only the discrete symmetries known as parity and charge conjugation. Parity is an inversion of the space coordinates and charge conjugation is an exchange of a particle with it's anti-particle. 
We wish to study an isovector meson $h$ with quantum numbers,

$$
J^{P C}=1^{-+}
$$

A set of suitable hybrid meson operators was proposed in [67], although for the purpose of computing its mass only. We adopt this choice here. The building blocks of the operators are

$$
\mathcal{O}_{h ; \nu}(t)=\sum_{\vec{x}} \bar{\psi}_{a}(\vec{x} t) \gamma_{\mu} \psi_{b}(\vec{x} t) F_{\mu \nu}^{a b}(\vec{x} t)
$$

where $\mu \neq \nu$ specifies a plane in the $d=4$ lattice, and $F_{\mu \nu}^{a b}$ is a sum of four products of $\mathrm{SU}(3)$ link matrices around clover leaf type paths centered at position $\vec{x}$. This is depicted in Fig. 2. Specifically we have,

$$
\begin{aligned}
F_{\mu \nu}(x) & =U_{\mu}(x) U_{\nu}(x+\hat{\mu}) U_{\mu}^{\dagger}(x+\hat{\nu}) U_{\nu}^{\dagger}(x) \\
& +U_{\nu}(x) U_{\mu}^{\dagger}(x-\hat{\mu}+\hat{\nu}) U_{\nu}^{\dagger}(x-\hat{\mu}) U_{\mu}(x-\hat{\mu}) \\
& +U_{\mu}^{\dagger}(x-\hat{\mu}) U_{\nu}^{\dagger}(x-\hat{\mu}-\hat{\nu}) U_{\mu}(x-\hat{\mu}-\hat{\nu}) U_{\nu}(x-\hat{\nu}) \\
& +U_{\nu}^{\dagger}(x-\hat{\nu}) U_{\mu}(x-\hat{\nu}) U_{\nu}(x+\hat{\mu}-\hat{\nu}) U_{\mu}^{\dagger}(x)
\end{aligned}
$$

One may distinguish between magnetic type gluons $\mu, \nu=1,2,3$ and electric type gluons with $\nu=4$ or $\mu=4$.

For the decay channel $\pi a_{1}$ a possible set of two-meson operators is

$$
\mathcal{O}_{\pi a_{1} ; k, \vec{r}}(t)=\sum_{\vec{x}} \sum_{\vec{y}} \delta_{\vec{x}-\vec{y}, \vec{r}} \bar{\psi}_{a}(\vec{x} t) \gamma_{5} \psi_{a}(\vec{x} t) \bar{\psi}_{b}(\vec{y} t) \gamma_{5} \gamma_{k} \psi_{b}(\vec{y} t)
$$

with $k=1,2,3$. The simplest choice for the relative distance is $\vec{r}=0$, which we will adopt in this project. Choices of $\vec{r} \neq 0$ would require projections on irreps of the discrete octahedral 3-d symmetry group. Although this is not a problem 


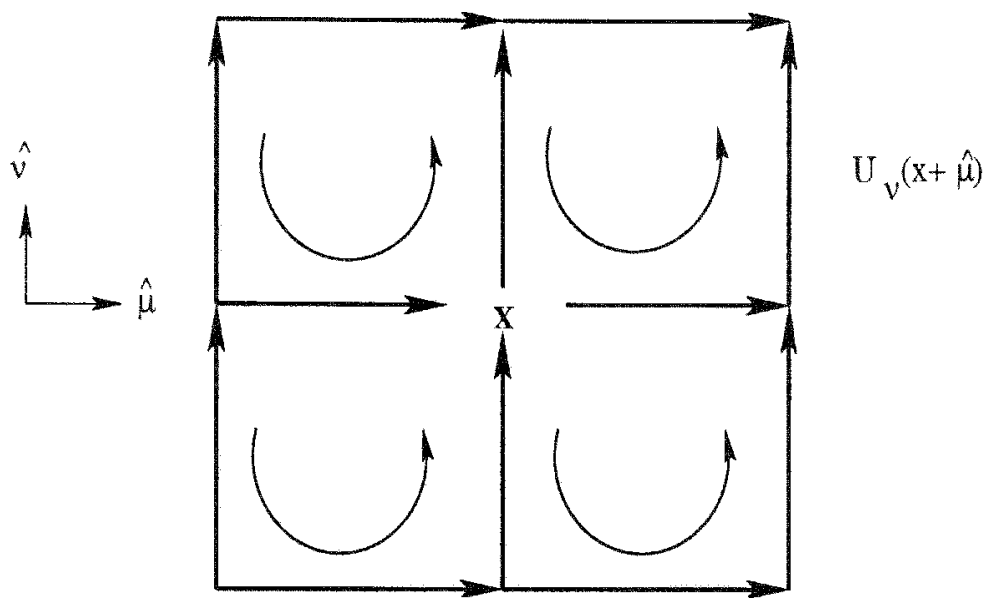

Figure 2: Depiction of the valence gluon field, eq. (3.3), used in the hybrid exotic operator. The loops represent the gluon field. Space-space planes are color magnetic fields and space-time planes are color electric fields.

in principle, the increased number of sources needed for fermion matrix inversions would be a considerable burden on available computing resources. Thus consider,

$$
\mathcal{O}_{\pi a_{1} ; k}(t)=\sum_{\vec{x}} \bar{\psi}_{a}(\vec{x} t) \gamma_{5} \psi_{a}(\vec{x} t) \cdot \bar{\psi}_{b}(\vec{y} t) \gamma_{5} \gamma_{k} \psi_{b}(\vec{y} t)
$$

The quark flavor structure of $\mathcal{O}_{h}$ and $\mathcal{O}_{\pi a_{1} ; k}$ still needs to be determined. For $\mathcal{O}_{h}$ we arbitrarily choose $\bar{\psi} \psi \sim \bar{d} u$. Thus $\mathcal{O}_{h^{+}}$describes a positively charged isovector meson $h^{+}$, with $I=1, I_{3}=+1$. Both $\pi$ and $a_{1}$ are isovector mesons, thus a state with exactly matching isospin quantum numbers is given by $\pi^{+} a_{1}^{0}-\pi^{0} a_{1}^{+}$(see Appendix F). Neglecting effects of $S U(2)$ isospin symmetry breaking on the decay width, we may retain only the first term $\pi^{+} a_{1}^{0}$ for constructing the operator $\mathcal{O}_{\pi a_{1} ; k}$. The corresponding quark flavor structure (see Appendix F) to be employed in $\mathcal{O}_{\pi a_{1} ; k}$ is $\bar{\psi} \psi \cdot \bar{\psi} \psi \sim \bar{d} u \cdot(\bar{d} d+\bar{u} u)$, see eq. (3.4). Hence $\mathcal{O}_{\pi a_{1} ; k}$ becomes,

$$
\begin{aligned}
& O_{\pi^{+} a_{1}^{0} ; k}(t)=\sum_{\vec{x}}\left[\bar{d}_{a}(\vec{x} t) \gamma_{5} u_{a}(\vec{x} t) \cdot \bar{d}_{b}(\vec{x} t) \gamma_{5} \gamma_{k} d_{b}(\vec{x} t)\right. \\
& \left.+\bar{d}_{a}(\vec{x} t) \gamma_{5} u_{a}(\vec{x} t) \cdot \bar{u}_{b}(\vec{x} t) \gamma_{5} \gamma_{k} u_{b}(\vec{x} t)\right] .
\end{aligned}
$$


Besides coupling to $I=1$, it also couples to $I=2$ channels. In the lattice simulation, however, we expect that excitations of states with $I \neq 1$ are suppressed because of the projecting effect of the "clean" $I=1$ operator $O_{h+}$ at the sink, as present in the off-diagonal elements of the correlation matrix.

With isospin components defined, next we verify parity and charge conjugation quantum numbers. Under parity transformation $\mathcal{P}$ the quark fields transform as [2],

$$
\begin{aligned}
& \mathcal{P} \psi(\vec{x} t) \mathcal{P}^{-1}=\gamma_{4} \psi(-\vec{x} t) \\
& \mathcal{P} \bar{\psi}(\vec{x} t) \mathcal{P}^{-1}=\bar{\psi}(-\vec{x} t) \gamma_{4}
\end{aligned}
$$

and the $\mathrm{SU}(3)$ gauge fields tranform as,

$$
\begin{aligned}
\mathcal{P} U_{i}(\vec{x} t) \mathcal{P}^{-1} & =U_{-i}(-\vec{x} t)=U_{i}^{\dagger}(x-\hat{i} t), \quad i=1,2,3 \\
\mathcal{P} U_{4}(\vec{x} t) \mathcal{P}^{-1} & =U_{4}(-\vec{x} t) .
\end{aligned}
$$

The hybrid operator, eq. (3.2), for magnetic type gluons, $1 \leq i, j<4$,

$$
O_{h^{+} ; j}(t)=\sum_{\vec{x}} \bar{d}_{a}(\vec{x} t) \gamma_{i} u_{b}(\vec{x} t) F_{i j}^{a b}(\vec{x} t)
$$

transforms under parity as,

$$
\begin{aligned}
& \mathcal{P} O_{h^{+} ; j}(t) \mathcal{P}^{-1}=\sum_{\vec{x}} \bar{d}(-\vec{x} t) \gamma_{4} \gamma_{j} \gamma_{4} u(-\vec{x} t) \\
& {\left[U_{-i}(-\vec{x} t) U_{-j}(-\vec{x}+\hat{i}, t) U_{-i}^{\dagger}(-\vec{x}+\hat{j}, t) U_{-j}^{\dagger}(-\vec{x} t)\right.} \\
+ & U_{-j}(-\vec{x} t) U_{-i}^{\dagger}(-\vec{x}-\hat{i}+\hat{j}, t) U_{-j}^{\dagger}(-\vec{x}-\hat{i}, t) U_{-i}(-\vec{x}-\hat{i}, t) \\
+ & U_{-i}^{\dagger}(-\vec{x}-\hat{i}, t) U_{-j}^{\dagger}(-\vec{x}-\hat{i}-\hat{j}, t) U_{-i}(-\vec{x}-\hat{i}-\hat{j}, t) U_{-j}(-\vec{x}-\hat{j}, t) \\
+ & \left.U_{-j}^{\dagger}(-\vec{x}-\hat{j}, t) U-i(-\vec{x}-\hat{j}, t) U_{-j}(-\vec{x}+\hat{i}-\hat{j}, t) U_{-i}^{\dagger}(-\vec{x} t)\right] .
\end{aligned}
$$


Changing the summation variable $\vec{x}$ to $-\vec{x}$, using periodicity, and recognizing that $F_{i j}$ is a sum over all quadrants in all spatial planes leaves the above expression invariant except that $\gamma_{4} \gamma_{j} \gamma_{4}=-\gamma_{j}$. Thus we have overall negative parity as desired,

$$
\mathcal{P}\left(O_{h^{+} ; j}(t)\right) \mathcal{P}^{-1}=-O_{h^{+} ; j}(t)
$$

The $\pi a_{1}$ operator eq. (3.6) transforms under parity as

$$
\begin{aligned}
& \mathcal{P} O_{\pi^{+} a_{1}^{0} ; k}(t) \mathcal{P}^{-1}= \\
& +\sum_{\vec{x}}\left(\bar{d}(-\vec{x} t) \gamma_{4} \gamma_{5} \gamma_{4} u(-\vec{x} t)\right) \cdot \bar{d}(-\vec{x} t) \gamma_{4} \gamma_{5} \gamma_{k} \gamma_{4} d(-\vec{x} t) \\
& +\sum_{\vec{x}}\left(\bar{d}(-\vec{x} t) \gamma_{4} \gamma_{5} \gamma_{4} u(-\vec{x} t)\right) \cdot \bar{u}(-\vec{x} t) \gamma_{4} \gamma_{5} \gamma_{k} \gamma_{4} u(-\vec{x} t) \\
& =\sum_{\vec{x}}\left(-\bar{d}(-\vec{x} t) \gamma_{5} u(-\vec{x} t)\right) \cdot \bar{d}(-\vec{x} t) \gamma_{5} \gamma_{k} d(-\vec{x} t) \\
& +\sum_{\vec{x}}\left(-\bar{d}(-\vec{x} t) \gamma_{5} u(-\vec{x} t)\right) \cdot \bar{u}(-\vec{x} t) \gamma_{5} \gamma_{k} u(-\vec{x} t) \\
& =-O_{\pi+a_{1}^{0} ; k}(t)
\end{aligned}
$$

provided that $k=1,2,3$. Hence, the $\pi a_{1}$ system has negative parity as desired. Under charge conjugation $\mathcal{C}$ the following transformations apply [2].

$$
\begin{gathered}
\mathcal{C} \psi(\vec{x}, t) \mathcal{C}^{-1}=C \bar{\psi}^{T}(\vec{x}, t) \\
\mathcal{C} \bar{\psi}(\vec{x}, t) \mathcal{C}^{-1}=-\psi^{T}(\vec{x}, t) C^{-1} \\
\mathcal{C} U_{i}(\vec{x}, t) \mathcal{C}^{-1}=U_{i}^{*}(\vec{x}, t) \\
\mathcal{C} U_{4}(\vec{x}, t) \mathcal{C}^{-1}=U_{4}^{*}(\vec{x}, t)
\end{gathered}
$$

where $C$ is the charge conjugation matrix (Appendix C) satisfying,

$$
C^{-1}=-C, \quad C^{-1} \gamma_{\mu} C=-\gamma_{\mu}^{T}, \quad \text { and } \quad C^{-1} \gamma_{5} C=\gamma_{5}^{T}
$$


Transforming the hybrid operator eq. (3.2) with either $\bar{\psi} \psi \sim \bar{d} d$ or $\bar{\psi} \psi \sim \bar{u} u$ gives for the magnetic case, $1 \leq i, j \leq 3$,

$$
\begin{aligned}
\mathcal{C O}_{h ; j}(t) \mathcal{C}^{-1} & =\sum_{\vec{x}} \mathcal{C} \bar{\psi}(\vec{x} t) \gamma_{i} \psi(\vec{x} t) F_{i j}(\vec{x} t) \mathcal{C}^{-1} \\
& =\sum_{\vec{x}}-\psi^{T}(\vec{x} t) C^{-1} \gamma_{i} C \bar{\psi}^{T}(\vec{x} t) F_{i j}^{*}(\vec{x} t) \\
& =\sum_{\vec{x}}+\psi^{T}(\vec{x} t) \gamma_{i}^{T} \bar{\psi}^{T}(\vec{x} t) F_{i j}^{*}(\vec{x} t) \\
& =\sum_{\vec{x}}-\left(\bar{\psi}(\vec{x} t) \gamma_{i} \psi(\vec{x} t)^{T}\right) F_{i j}^{\dagger}(\vec{x} t) \\
& =\sum_{\vec{x}}\left(-\bar{\psi}(\vec{x} t) \gamma_{i} \psi(\vec{x} t)\right) F_{i j}^{\dagger}(\vec{x} t) \\
& =-\mathcal{O}_{h^{\prime} ; j}(t) .
\end{aligned}
$$

Therefore, to create positive charge conjugation, it is necessary to subtract the adjoint of $F_{i j}[67]$, arriving at,

$$
\begin{gathered}
\mathcal{C}\left(\mathcal{O}_{h}-\mathcal{O}_{h^{\prime}}\right) \mathcal{C}^{-1}=-\mathcal{O}_{h^{\prime}}-\left(-\mathcal{O}_{h}\right)=\mathcal{O}_{h}-\mathcal{O}_{h^{\prime}} \\
\mathcal{O}_{h ; j}(t)-\mathcal{O}_{h^{\prime} ; j}(t)=\sum_{\vec{x}} \bar{\psi}(\vec{x} t) \gamma_{i} \psi(\vec{x} t)\left[F_{i j}(\vec{x} t)-F_{i j}^{\dagger}(\vec{x} t)\right] .
\end{gathered}
$$

Thus finally, the hybrid meson operator used for the $h^{+}$in this work is

$$
O_{h}(t)=\sum_{1 \leq i<j \leq 3} \sum_{\vec{x}} \vec{d}_{a}(\vec{x} t) \gamma_{i} u_{b}(\vec{x} t)\left[F_{i j}^{a b}(\vec{x} t)-F_{i j}^{\dagger a b}(\vec{x} t)\right]
$$

which includes the sum over all spatial planes. 
Applying charge conjugation to the $\pi a_{1}$ system from eq. (3.6) (letting $d=d(\vec{x} t)$, etc.),

$$
\begin{aligned}
\mathcal{C} O_{\pi^{+} a_{1}^{0} ; k}(t) \mathcal{C}^{-1} & =\sum_{\vec{x}}\left(\left(d^{T} C^{-1} \gamma_{5} C \bar{u}^{T}\right)\left(-d^{T} C^{-1} \gamma_{5} \gamma_{k} C \bar{d}^{T}-u^{T} C^{-1} \gamma_{5} \gamma_{k} C \bar{d}^{T}\right)\right) \\
& =\sum_{\vec{x}}\left(\left(-d^{T} \gamma_{5}^{T} \bar{u}^{T}\right)\left(+d^{T} \gamma_{5}^{T} \gamma_{k}^{T} \bar{d}^{T}+u^{T} \gamma_{5}^{T} \gamma_{k}^{T} \bar{d}^{T}\right)\right) \\
& =\sum_{\vec{x}}\left(\left(+\bar{u} \gamma_{5} d\right)\left(\bar{d} \gamma_{5} \gamma_{k} d+\bar{u} \gamma_{5} \gamma_{k} u\right)\right) \\
& =O_{\pi^{-} a_{1}^{0} ; k}(t) .
\end{aligned}
$$

Therefore, the $\pi a_{1}$ operator has the desired charge conjugation property.

Including a sum over spatial directions, finally, the operator

$$
O_{\pi a_{1}}(t)=\sum_{k=1}^{3} O_{\pi^{+} a_{1}^{0} ; k}(t)
$$

for the $\pi^{+} a_{1}^{0}$ is used in this work.

\subsection{Equal time correlators.}

The operators eq's (3.29) and (3.31) are the basis for calculating correlation functions

$$
C_{X Y}\left(t, t_{0}\right)=<O_{X}(t) O_{Y}^{\dagger}\left(t_{0}\right)>-<O_{X}(t)><O_{Y}^{\dagger}\left(t_{0}\right)>
$$

where $X$ and $Y$, standing for $h$ and $\pi a_{1}$, establish a $2 \times 2$ correlation matrix. The separable terms in eq. (3.32) are zero because of the quark flavor assignment in $X=h$ and $Y=\pi a_{1}$. Specifically, showing the flavor stuctrure only, from eqs. (3.29) and (3.6) we have,

$$
<O_{h}(t)>\sim<\vec{u} d>=0
$$




$$
<O_{\pi a_{1}}(t)>\sim<\bar{d} u \bar{d} d+\bar{d} u \bar{u} u>=0 .
$$

In each of the above expectation values there is a factor of a contraction between unlike quark fields which is zero. Similar statements hold for the $O^{\dagger}$ operators. Thus the correlation matrix has the form

$$
C\left(t, t_{0}\right)=\left(\begin{array}{cc}
\left.<O_{h}(t) O_{h}^{\dagger}\left(t_{0}\right)\right\rangle & <O_{h}(t) O_{\pi a_{1}}^{\dagger}\left(t_{0}\right)> \\
\left.<O_{\pi a_{1}}(t) O_{h}^{\dagger}\left(t_{0}\right)\right\rangle & <O_{\pi a_{1}}(t) O_{\pi \alpha_{1}}^{\dagger}\left(t_{0}\right)>
\end{array}\right) .
$$

The remaining (nonseparable) terms in eq. (3.35) contain contractions between quark fields at equal times when worked out with Wick's theorem, cf. (2.42). For example, showing flavor structure only,

$$
C_{h, \pi a_{1}}\left(t, t_{0}\right) \sim<(\bar{u} d)_{t} \cdot(\bar{d} d \bar{u} d+\bar{u} u \bar{u} d)_{t_{0}}>
$$

with time arguments $t$ and $t_{0}$ as indicated. There occur equal-time contractions : $d \vec{d}$ : and : $u \vec{u}$ : at time $t_{0}$ only. The corresponding propagator elements $Q^{-1}\left(\vec{x} t_{0}, \vec{y} t_{0}\right)$ are calculated by default and thus pose no computational problem. This is different for

$$
C_{\pi a_{1}, \pi a_{1}}\left(t, t_{0}\right) \sim<(\bar{d} u \bar{d} d+\bar{d} u \bar{u} u)_{t} \cdot(\bar{d} d \bar{u} d+\bar{u} u \bar{u} d)_{t_{0}}>
$$

where we encounter equal time contractions $d \bar{d}$ and $u \bar{u}$ at time $t>t_{0}$. Computing $Q^{-1}(\vec{x} t, \vec{y} t)$ is very resource intensive because sources have to be placed at all time slices $t$. We avoid this problem by, first, noting that at equal $m_{d}=m_{u}$ the contractions $d \bar{d}$ and $u \bar{u}$ give rise to the same propagator $Q^{-1}(\vec{x} t, \vec{y} t)$. Thus, replacing the $(\ldots)_{t}$ term in eq. (3.37) by $(2 \bar{d} u \bar{d} d)_{t}$ and reinstating the $\gamma$-matrices we observe that $\bar{d} d \sim \bar{d} \gamma_{5} \gamma_{k} d$ is an $a_{1}$ meson operator. We now argue that altering the flavor of the $d$ quark to an $s$ quark has no significant effect on the mass spectra. The reason is 
that this substitution essentially changes the $a_{1}$ into a $K_{1}$ meson

$$
a_{1}=\bar{d} \gamma_{5} \gamma_{k} d \longrightarrow \bar{d} \gamma_{5} \gamma_{k} s=K_{1}
$$

Because the masses of the $a_{1}(1230 \mathrm{MeV})$ and $\mathscr{A}_{1}(1270 \mathrm{MeV})$ are so close [3], this substitution should have little effect on the mass spectrum of the $\pi a_{1}$ system, and ultimately, on the resulting decay width. Thus, after the substitution eq. (3.37) becomes,

$$
C_{\pi a_{1}, \pi a_{1}}\left(t, t_{0}\right) \sim<(2 \bar{d} u \bar{d} s)_{t}(2 \bar{s} d \bar{u} d)_{t_{0}}>
$$

Here no equal time contractions occur. Note that the substitution must be done for both $\mathcal{O}$ and $O^{\dagger}$ to preserve Hermiticity of the correlation matrix.

Based upon the above argument, we adopt the approximation that all equal time contractions may be neglected in calculating $C_{\pi a_{1}, \pi a_{1}}\left(t, t_{0}\right)$ from the specific

operators $O_{\pi a_{1}}$ and $O_{\pi a_{1}}^{\dagger}$ as written in eqs. (3.31) and (3.6). All other matrix elements are not effected.

Finally, we do not explicitly compute $C_{\pi a_{1}, h}\left(t, t_{0}\right)$ because it can be inferred from the hermiticity of the correlation matrix.

\subsection{Forming the contractions and propagators}

Knowing the operators for $h^{+} \rightarrow \pi^{+} a_{1}^{0}$, the actual correlation functions are calculated by contracting the various quark fields into propagators and multiplying the various gamma matrices out to see which elements are non-zero. For example, the $\pi a_{1}$ component to the correlation matrix is calculated as follows. The two $\pi$ 
operators needed for this are,

$$
O_{\pi+}(t)=\frac{1}{\sqrt{V}} \sum_{\vec{x}} \bar{d}_{a \mu}(\vec{x} t) \gamma_{5_{\mu \nu}} u_{a \nu}(\vec{x} t)
$$

and

$$
O_{\pi^{+}}^{\dagger}(t)=\frac{1}{\sqrt{V}} \sum_{\vec{x}} \bar{u}_{a \nu}(\vec{x} t) \bar{\gamma}_{5_{\nu \mu}} d_{a \mu}(\vec{x} t)
$$

where $\bar{\gamma}=\gamma^{\dagger} \gamma_{4}$

The two $a_{1}$ operators needed are,

$$
O_{a_{1}^{0}}(t)=\frac{1}{\sqrt{V}} \sum_{\vec{x}}\left(\bar{d}_{b \alpha}(\vec{x} t)\left(\gamma_{5} \gamma_{k}\right)_{\alpha \beta} d_{b \beta}(\vec{x} t)+\vec{u}_{b \alpha}(\vec{x} t)\left(\gamma_{5} \gamma_{k}\right)_{\alpha \beta} u_{b \beta}(\vec{x} t)\right)
$$

and

$$
O_{a_{1}^{0}}^{\dagger}(t)=\frac{1}{\sqrt{V}} \sum_{\vec{x}}\left(\bar{d}_{b \beta}(\vec{x} t)\left(\bar{\gamma}_{5} \bar{\gamma}_{k}\right)_{\beta \alpha} d_{b \alpha}(\vec{x} t)+\bar{u}_{b \beta}(\vec{x} t)\left(\bar{\gamma}_{5} \bar{\gamma}_{k}\right)_{\beta \alpha} u_{b \alpha}(\vec{x} t)\right)
$$

The Dirac indices $\mu, \nu, \alpha, \beta$, etc. are now explicitly shown and a normalization to the lattice volume $V$ is included.

Note that the $d$ flavor is retained in all operators. The $d \rightarrow s$ flavor substitution was only used in the argument to justify removing equal time contractions, as discussed in Section 3.2. The combined $\pi a_{1}$ operator is then,

$$
\begin{aligned}
& O_{\pi a_{1}}(t)=\frac{1}{\sqrt{V}} \sum_{\vec{x}} \\
& \bar{d}_{a \mu}(\vec{x} t) \gamma_{5 \mu \nu} u_{a \nu}(\vec{x} t) \cdot \bar{d}_{b \alpha}(\vec{x} t)\left(\gamma_{5} \gamma_{k}\right)_{\alpha \beta} d_{b \beta}(\vec{x} t)
\end{aligned}
$$

The correlation matrix element becomes,

$$
\begin{aligned}
& <O_{\pi a_{1}}(t) O_{\pi a_{1}}^{\dagger}(t)>=<\frac{1}{V} \sum_{\vec{x}} \sum_{\vec{x}^{\prime}} \\
& \bar{d}_{a \mu}(\vec{x} t) \gamma_{5 \mu \nu} u_{a \nu}(\vec{x} t) \bar{d}_{b \alpha}(\vec{x} t)\left(\gamma_{5} \gamma_{k}\right)_{\alpha \beta} d_{b \beta}(\vec{x} t) \\
& \bar{d}_{b^{\prime} \beta^{\prime}}\left(\vec{x}^{\prime} t\right)\left(\bar{\gamma}_{5} \bar{\gamma}_{k^{\prime}}\right)_{\beta^{\prime} \alpha^{\prime}} d_{b^{\prime} \alpha^{\prime}}\left(\overrightarrow{x^{\prime}} t\right) \bar{u}_{a^{\prime} \nu^{\prime}}\left(\overrightarrow{x^{\prime} t}\right) \bar{\gamma}_{5 \nu^{\prime} \mu^{\prime}} d_{a^{\prime} \mu}\left(\overrightarrow{\left.x^{\prime} t\right)>.}\right.
\end{aligned}
$$


So the matrix element becomes

$$
\begin{aligned}
& <O_{\pi a_{1}}(t) O_{\pi a_{1}}^{\dagger}(t)>=<\frac{1}{V} \sum_{\vec{x}} \sum_{\vec{x}^{\prime}} \gamma_{5 \mu \nu}\left(\gamma_{5} \gamma_{k}\right)_{\alpha \beta}\left(\overline{\gamma_{5}} \bar{\gamma}_{k^{\prime}}\right)_{\beta^{\prime} \alpha^{\prime}} \bar{\gamma}_{5 \nu^{\prime} \mu^{\prime}} \\
& u_{a \nu}(\vec{x} t) \bar{u}_{a^{\prime} \nu^{\prime}}\left(\vec{x}^{\prime} t_{0}\right) d_{b \beta}(\vec{x} t) d_{b^{\prime} \alpha^{\prime}} \\
& \left(\vec{x}^{\prime} t_{0}\right) d_{a^{\prime} \mu^{\prime}}\left(\vec{x}^{\prime} t_{0}\right) \bar{d}_{a \mu}(\vec{x} t) \bar{d}_{b \alpha}(\vec{x} t) \bar{d}_{b^{\prime} \beta^{\prime}}\left(\vec{x}^{\prime} t_{0}\right)>.
\end{aligned}
$$

A contraction gives an inverse element of the fermion matrix, see eq. (2.33). For convenience write $G=Q^{-1}$ then,

$$
: \psi_{a \mu}(\vec{x} t) \bar{\psi}_{b \nu}\left(\vec{x}^{\prime} t_{0}\right):=G_{a \mu, b \nu}\left(\vec{x} t, \vec{x}^{\prime} t_{0}\right)
$$

Where $G$ is the propagator (amplitude) for the quark field at $\left(\vec{x}^{\prime} t_{0}\right)$ with color/spin properties $b \nu$ to move to $(\vec{x} t)$ and have color/spin properties $a \mu$. For example,

$$
: d_{b \beta}(\vec{x} t) \bar{d}_{b^{\prime} \beta^{\prime}}\left(\vec{x}^{\prime} t_{0}\right):=G_{b \beta, b^{\prime} \beta^{\prime}}\left(\vec{x} t, \vec{x}^{\prime} t_{0}\right)
$$

For the correlator $C_{\pi a_{1}, \pi a_{1}}\left(t, t_{0}\right)$, all contractions between equal time quark fields are set to zero as discussed in Section 3.2:

$$
G_{b \beta, a \alpha}(\vec{y} t, \vec{x} t)=0
$$

After doing all the remaining contractions, this matrix element reduces to two terms with four propagators each, along with the gamma matrix structure.

$$
\begin{gathered}
<O_{\pi a_{1}}(t) O_{\pi a_{1}}^{\dagger}\left(t_{0}\right)>= \\
<\frac{1}{V} \sum_{\vec{x}} \sum_{\vec{x}^{\prime}} \gamma_{5 \mu \nu}\left(\gamma_{5} \gamma_{k}\right)_{\alpha \beta}\left(\overline{\gamma_{5}} \overline{\gamma_{k^{\prime}}}\right)_{\beta^{\prime} \alpha^{\prime}} \overline{\gamma_{5 \nu^{\prime} \mu^{\prime}}} \\
{\left[G_{a \nu^{\prime} \alpha^{\prime} \nu^{\prime}}\left(\vec{x} t, \vec{x}^{\prime} t_{0}\right) G_{a^{\prime} \mu^{\prime}, a \mu}\left(\vec{x}^{\prime} t_{0}, \vec{x} t\right)\right.} \\
G_{b^{\prime} \alpha^{\prime}, b \alpha}\left(\vec{x}^{\prime} t_{0}, \vec{x} t\right) G_{b \beta, b^{\prime} \beta^{\prime}}\left(\vec{x} t, \vec{x}^{\prime} t_{0}\right)
\end{gathered}
$$




$$
\begin{aligned}
& -G_{a \nu^{\prime} a^{\prime} \nu^{\prime}}\left(\vec{x} t, \vec{x}^{\prime} t_{0}\right) G_{a^{\prime} \mu^{\prime}, b \alpha}\left(\vec{x}^{\prime} t_{0}, \vec{x} t\right) \\
& \left.G_{b^{\prime} \alpha^{\prime}, a \mu^{\prime}}\left(\vec{x}^{\prime} t_{0}, \vec{x} t\right) G_{b \beta, b^{\prime} \beta^{\prime}}\left(\vec{x} t, \vec{x}^{\prime} t_{0}\right)\right]>.
\end{aligned}
$$

If the approximation to drop equal time propagators was not made, then four more terms would be needed for this matrix element. Factoring out one of the propagators yields,

$$
\begin{gathered}
<O_{\pi a_{1}}(t) O_{\pi a_{1}}^{\dagger}\left(t_{0}\right)>= \\
<\frac{1}{V} \sum_{\vec{x}} \sum_{\vec{x}^{\prime}} \gamma_{5 \mu \nu}\left(\gamma_{5} \gamma_{k}\right)_{\alpha \beta}\left(\overrightarrow{\gamma_{5}} \bar{\gamma}_{k^{\prime}}\right)_{\beta^{\prime} \alpha^{\prime}} \bar{\gamma}_{5 \nu^{\prime} \mu^{\prime}} \\
G_{a \nu^{\prime} a^{\prime} \nu^{\prime}}\left(\vec{x} t, \vec{x}^{\prime} t_{0}\right)\left[G_{a^{\prime} \mu^{\prime}, A \mu}\left(\vec{x}^{\prime} t_{0}, \vec{x} t\right) G_{b^{\prime} \alpha^{\prime}, b \alpha}\left(\vec{x}^{\prime} t_{0}, \vec{x} t\right)\right. \\
\left.-G_{b^{\prime} \mu^{\prime}, b \alpha}\left(\vec{x}^{\prime} t_{0}, \vec{x} t\right) G_{b^{\prime} \alpha^{\prime}, A \mu^{\prime}}\left(\vec{x}^{\prime} t_{0}, \vec{x} t\right)\right] G_{b \beta, b^{\prime} \beta^{\prime}}\left(\vec{x} t, \vec{x}^{\prime} t_{0}\right)>.
\end{gathered}
$$

Now we apply the property of translational invariance on the lattice by letting $\vec{x} \rightarrow \vec{x}+\vec{x}^{\prime}$ and shift by an amount $\vec{x}^{\prime}$ so that $\vec{x}^{\prime} \rightarrow 0$. The matrix element becomes,

$$
\begin{gathered}
<O_{\pi a_{1}}(t) O_{\pi a_{1}}^{\dagger}\left(t_{0}\right)>= \\
<\sum_{\vec{x}} \gamma_{5 \mu \nu}\left(\gamma_{5} \gamma_{k}\right)_{\alpha \beta}\left(\bar{\gamma}_{5} \bar{\gamma}_{k^{\prime}}\right)_{\beta^{\prime} \alpha^{\prime}} \bar{\gamma}_{5 \nu^{\prime} \mu^{\prime}} \\
G_{a \nu^{\prime} a^{\prime} \nu^{\prime}}\left(\vec{x} t, \overrightarrow{0} t_{0}\right)\left[G_{a^{\prime} \mu^{\prime}, a \mu}\left(\overrightarrow{0} t_{0}, \vec{x} t\right) G_{b^{\prime} \alpha^{\prime}, b \alpha}\left(\overrightarrow{0} t_{0}, \vec{x} t\right)\right. \\
\left.-G_{a^{\prime} \mu^{\prime}, b \alpha}\left(\overrightarrow{0} t_{0}, \vec{x} t\right) G_{b^{\prime} \alpha^{\prime}, a \mu^{\prime}}\left(\overrightarrow{0} t_{0}, \vec{x} t\right)\right] \\
G_{b \beta, b^{\prime} \beta^{\prime}}\left(\vec{x} t, \overrightarrow{0} t_{0}\right)>.
\end{gathered}
$$

Using the relation,

$$
G_{a \mu, b \nu}(y, x)=\gamma_{5 \mu \mu^{\prime}} G_{b \nu^{\prime}, a \mu^{\prime}}^{*}(x, y) \gamma_{5 \nu^{\prime} \nu}
$$

allows us to rewrite the matrix element as,

$$
<O_{\pi a_{1}}(t) O_{\pi a_{1}}^{\dagger}\left(t_{0}\right)>=
$$




$$
\begin{gathered}
<\sum_{\vec{x}} \gamma_{5 \mu \nu}\left(\gamma_{5} \gamma_{k}\right)_{\alpha \beta}\left(\vec{\gamma}_{5} \bar{\gamma}_{k^{\prime}}\right)_{\beta^{\prime} \alpha^{\prime}} \vec{\gamma}_{5 \nu^{\prime} \mu^{\prime}} \\
G_{a \nu, a^{\prime} \nu^{\prime}}\left(\vec{x} t, \overrightarrow{0} t_{0}\right) G_{b \beta, b^{\prime} \beta^{\prime}}\left(\vec{x} t, \overrightarrow{0} t_{0}\right) \\
{\left[\gamma_{5 \mu^{\prime} \mu^{\prime \prime \prime}} G_{a^{\prime} \mu^{\prime \prime}, a^{\prime} \mu^{\prime \prime \prime}}^{*}\left(\vec{x} t, \overrightarrow{0} t_{0}\right) \gamma_{5 \mu^{\prime \prime} \mu} \gamma_{5 \alpha^{\prime} \alpha^{\prime \prime \prime}}\right.} \\
G_{b^{\prime} \alpha^{\prime \prime}, b^{\prime} \alpha^{\prime \prime \prime}}^{*}\left(\vec{x} t, \overrightarrow{0} t_{0}\right) \gamma_{5 \alpha^{\prime \prime} \alpha} \\
-\gamma_{5 \mu^{\prime} \mu^{\prime \prime \prime}} G_{b \alpha^{\prime \prime}, a^{\prime} \mu^{\prime \prime \prime}}\left(\vec{x} t, \overrightarrow{0} t_{0}\right) \gamma_{5 \alpha^{\prime \prime} \alpha} \gamma_{5 \alpha^{\prime} \alpha^{\prime \prime \prime}} \\
\left.G_{a \mu^{\prime \prime}, b^{\prime} \alpha^{\prime \prime \prime}}\left(\vec{x} t, \overrightarrow{0} t_{0}\right) \gamma_{5, k^{\prime \prime} \mu}\right]>
\end{gathered}
$$

Pulling out all the gamma matrices and reducing their product gives, for one of the two terms,

$$
\begin{gathered}
\gamma_{5 \mu \nu}\left(\gamma_{5} \gamma_{k}\right)_{\alpha \beta}\left(\bar{\gamma}_{5} \bar{\gamma}_{k^{\prime}}\right)_{\beta^{\prime} \alpha^{\prime}} \bar{\gamma}_{5 \nu^{\prime} \mu^{\prime}} \gamma_{5 \mu^{\prime} \mu^{\prime \prime \prime}} \gamma_{5 \mu^{\prime \prime} \mu} \gamma_{5 \alpha^{\prime} \alpha^{\prime \prime \prime}} \gamma_{5 \alpha^{\prime \prime} \alpha} \\
=\gamma_{k_{\alpha^{\prime \prime} \beta}} \bar{\gamma}_{k_{\beta^{\prime} \alpha^{\prime \prime \prime}}^{\prime}} \bar{\gamma}_{5 \nu^{\prime} \mu^{\prime}} \gamma_{5_{\mu^{\prime} \mu^{\prime \prime \prime}}} \delta_{\nu \mu^{\prime \prime}} \\
=\gamma_{k_{\alpha^{\prime \prime} \beta}} \bar{\gamma}_{k_{\beta^{\prime} \alpha^{\prime \prime \prime}}^{\prime}}\left(-\delta_{\nu^{\prime} \mu^{\prime \prime \prime}}\right) \delta_{\nu \mu^{\prime \prime}}
\end{gathered}
$$

The other term has identical gamma matrix structure. Therefore, a final expression for this matrix element is (see Fig. 3),

$$
\begin{gathered}
<O_{\pi a_{1}}(t) O_{\pi a_{1}}^{\dagger}\left(t_{0}\right)>= \\
<\sum_{\vec{x}} G_{a \nu^{\prime} a^{\prime} \nu^{\prime}}\left(\vec{x} t, \overrightarrow{0} t_{0}\right) G_{b \beta, b^{\prime} \beta^{\prime}}\left(\vec{x} t, \overrightarrow{0} t_{0}\right) \\
{\left[G_{a, \mu^{\prime \prime}, a^{\prime} \mu^{\prime \prime \prime}}\left(\vec{x} t, \overrightarrow{0} t_{0}\right) G_{b \alpha^{\prime \prime}, b^{\prime} \alpha^{\prime \prime \prime}}^{*}\left(\vec{x} t, \overrightarrow{0} t_{0}\right)\right.} \\
\left.-G_{b \alpha^{\prime \prime}, a^{\prime} \mu^{\prime \prime \prime}}^{*}\left(\vec{x} t, \overrightarrow{0} t_{0}\right) G_{a \mu^{\prime \prime}, b^{\prime} \alpha^{\prime \prime \prime}}^{*}\left(\vec{x} t, \overrightarrow{0} t_{0}\right)\right] \\
\gamma_{k_{\alpha^{\prime \prime}, \beta}} \bar{\gamma}_{k_{\beta^{\prime} \alpha^{\prime \prime \prime}}^{\prime}}\left(-\delta_{\nu^{\prime} \mu^{\prime \prime \prime}}\right) \delta_{\nu \mu^{\prime \prime}}>. \\
=<\sum_{\vec{x}} G_{a \nu^{\prime \prime} a^{\prime} \nu^{\prime}}\left(\vec{x} t, \overrightarrow{0} t_{0}\right) G_{b \beta, b^{\prime} \beta^{\prime}}\left(\vec{x} t, \overrightarrow{0} t_{0}\right)
\end{gathered}
$$




$$
\begin{gathered}
{\left[G_{b \alpha^{\prime \prime}, a^{\prime} \nu^{\prime}}^{*}\left(\vec{x} t, \overrightarrow{0} t_{0}\right) G_{a \nu, b^{\prime} \alpha^{\prime \prime \prime}}^{*}\left(\vec{x} t, \overrightarrow{0} t_{0}\right)\right.} \\
\left.-G_{a \nu, a^{\prime} \nu^{\prime}}^{*}\left(\vec{x} t, \overrightarrow{0} t_{0}\right) G_{b \alpha^{\prime \prime}, b^{\prime} \alpha^{\prime \prime \prime}}^{*}\left(\vec{x} t, \overrightarrow{0} t_{0}\right)\right] \\
\gamma_{k_{\alpha^{\prime \prime} \beta}} \gamma_{k_{\beta^{\prime} \alpha^{\prime \prime \prime}}^{\prime}}>
\end{gathered}
$$

Following the same procedure as above, the matrix element for the $h$ to $\pi a_{1}$ transition can be calculated:

$$
\begin{gathered}
<O_{h}(t) O_{\pi a_{1}}^{\dagger}\left(t_{0}\right)>= \\
<\frac{1}{V} \sum_{\vec{x}} \sum_{\vec{y}} \gamma_{m_{\mu \nu}}\left(F_{m n}^{a b}(\vec{x} t)-F_{m n}^{\dagger a b}(\vec{x} t)\right) \\
\bar{d}_{a \mu}(\vec{x} t) u_{b \nu}(\vec{x} t) \bar{d}_{b^{\prime} \beta^{\prime}}\left(\vec{y} t_{0}\right)\left(\bar{\gamma}_{5} \bar{\gamma}_{n^{\prime}}\right)_{\beta^{\prime} \alpha^{\prime}} d_{b^{\prime} \alpha^{\prime}}\left(\vec{y} t_{0}\right) \\
\bar{u}_{a^{\prime} \nu^{\prime}}\left(\vec{y} t_{0}\right) \bar{\gamma}_{5_{\nu^{\prime} \mu^{\prime}}} d_{a^{\prime} \mu^{\prime}}\left(\vec{y} t_{0}\right)>.
\end{gathered}
$$

Moving the $\gamma$ matrices to the left and re-arranging the quark fields gives,

$$
\begin{gathered}
<O_{h}(t) O_{\pi a_{1}}^{\dagger}\left(t_{0}\right)>= \\
<\frac{1}{V} \sum_{\vec{x}} \sum_{\vec{x}^{\prime}} \gamma_{m \mu \nu}\left(\bar{\gamma}_{5} \bar{\gamma}_{n^{\prime}}\right)_{\beta^{\prime} \alpha^{\prime}} \bar{\gamma}_{5_{\nu^{\prime} \mu^{\prime}}}\left(F_{m n}^{a b}(\vec{x} t)-F_{m n}^{\dagger a b}(\vec{x} t)\right) \\
u_{b \nu}(\vec{x} t) \bar{u}_{a^{\prime} \nu^{\prime}}\left(\vec{x}^{\prime} t_{0}\right) d_{b^{\prime} \alpha^{\prime}}\left(\vec{x}^{\prime} t_{0}\right) d_{a^{\prime} \mu^{\prime}}\left(\vec{x}^{\prime} t_{0}\right) \\
\bar{d}_{a \mu}(\vec{x} t) \bar{d}_{b^{\prime} \beta^{\prime}}\left(\vec{x}^{\prime} t_{0}\right)>
\end{gathered}
$$

Performing the contractions and applying translational invariance leaves,

$$
\begin{gathered}
<O_{h}(t) O_{\pi a_{1}}^{\dagger}\left(t_{0}\right)>= \\
<\sum_{\vec{x}} \gamma_{m_{\mu \nu}}\left(\bar{\gamma}_{5} \bar{\gamma}_{n^{\prime}}\right)_{\beta^{\prime} \alpha^{\prime}} \bar{\gamma}_{5_{\nu^{\prime} \mu^{\prime}}}\left(F_{m n}^{a b}(\vec{x} t)-F_{m n}^{\dagger a b}(\vec{x} t)\right) \\
G_{b \nu, a^{\prime} \nu^{\prime}}\left(\vec{x} t, \overrightarrow{0} t_{0}\right)\left[G_{a^{\prime} \mu^{\prime}, a \mu}\left(\overrightarrow{0} t_{0}, \vec{x} t\right) G_{b^{\prime} \alpha^{\prime}, b^{\prime} \beta^{\prime}}\left(\overrightarrow{0} t_{0}, \overrightarrow{0} t_{0}\right)\right. \\
\left.-G_{a^{\prime} \mu^{\prime}, b^{\prime} \beta^{\prime}}\left(\overrightarrow{0} t_{0}, \overrightarrow{0} t_{0}\right) G_{b^{\prime} \alpha^{\prime}, a \mu}\left(\overrightarrow{0} t_{0}, \vec{x} t\right)\right]>.
\end{gathered}
$$


The two equal time propagators in this expression do not add much complexity because they are not summed over and are evaluated on one timeslice only. As before, we want propagators from $\overrightarrow{0}$ to $\vec{x}$. Applying eq. (3.53) the equation becomes,

$$
\begin{gathered}
<O_{h}(t) O_{\pi a_{1}}^{\dagger}\left(t_{0}\right)>= \\
\sum_{x} \gamma_{m_{\mu \nu}}\left(\bar{\gamma}_{5} \bar{\gamma}_{n^{\prime}}\right)_{\beta^{\prime} \alpha^{\prime}} \bar{\gamma}_{5_{\nu^{\prime} \mu^{\prime}}}\left(F_{m n}^{a b}(\vec{x} t)-F_{m n}^{\dagger a b}(\vec{x} t)\right) G_{b \nu, a^{\prime} \nu^{\prime}}\left(\vec{x} t, \overrightarrow{0} t_{0}\right) \\
{\left[\gamma_{5_{\mu^{\prime} \mu^{\prime \prime \prime}}} G_{a \mu^{\prime \prime}, a^{\prime} \mu^{\prime \prime \prime}}^{*}\left(\vec{x} t, \overrightarrow{0} t_{0}\right) \gamma_{5_{\mu^{\prime \prime} \mu^{\prime}}} G_{b^{\prime} \alpha^{\prime}, b^{\prime} \beta^{\prime}}\left(\overrightarrow{0} t_{0}, \overrightarrow{0} t_{0}\right)\right.} \\
\left.-G_{a^{\prime} \mu^{\prime}, b^{\prime} \beta^{\prime}}\left(\overrightarrow{0} t_{0}, \overrightarrow{0} t_{0}\right) \gamma_{5_{\alpha^{\prime} \alpha^{\prime \prime \prime}}} G_{a \mu^{\prime \prime}, b^{\prime} \alpha^{\prime \prime \prime}}^{*}\left(\vec{x} t, \overrightarrow{0} t_{0}\right) \gamma_{\bar{\mu}_{\mu^{\prime \prime} \mu}}\right]
\end{gathered}
$$

Now the gamma matrix structure can be reduced,

$$
\begin{aligned}
& \gamma_{m_{\mu \nu}}\left(\bar{\gamma}_{5} \bar{\gamma}_{n^{\prime}}\right)_{\beta^{\prime} \alpha^{\prime}} \bar{\gamma}_{5_{\nu^{\prime} \mu^{\prime}}} \gamma_{5_{\mu^{\prime} \mu^{\prime \prime \prime}}} \gamma_{\mu_{\mu^{\prime \prime} \mu}} \\
& =\gamma_{m_{\mu^{\prime \prime}}}\left(\bar{\gamma}_{5} \bar{\gamma}_{n^{\prime}}\right)_{\beta^{\prime} \alpha^{\prime}}\left(-\delta_{\nu^{\prime} \mu^{\prime \prime \prime}}\right) .
\end{aligned}
$$

Also,

$$
\begin{aligned}
& \gamma_{m_{\mu \nu}}\left(\bar{\gamma}_{5} \bar{\gamma}_{n^{\prime}}\right)_{\beta^{\prime} \alpha^{\prime}} \bar{\gamma}_{5_{\nu^{\prime} \mu^{\prime}}} \gamma_{5 \alpha^{\prime} \alpha^{\prime \prime \prime}} \gamma_{5 \mu^{\prime \prime} \mu} \\
& =\gamma_{m_{\mu^{\prime \prime} \nu}}\left(\bar{\gamma}_{5} \bar{\gamma}_{n^{\prime}}\right)_{\beta^{\prime \prime \prime} \alpha^{\prime \prime \prime}}\left(-\delta_{\nu^{\prime} \mu^{\prime \prime \prime}}\right) .
\end{aligned}
$$

A final expression for this matrix element is (see Fig. 3),

$$
\begin{gathered}
<O_{h}(t) O_{\pi a_{1}}^{\dagger}\left(t_{0}\right)>=<\sum_{\vec{x}} G_{b \nu, a^{\prime} \nu^{\prime}}\left(\vec{x} t, \overrightarrow{0} t_{0}\right) \\
{\left[G_{b^{\prime} \beta^{\prime \prime}, a^{\prime} \nu^{\prime}}^{*}\left(\overrightarrow{0} t_{0}, \overrightarrow{0} t_{0}\right) G_{a \mu^{\prime \prime}, b^{\prime} \alpha^{\prime \prime \prime}}^{*}\left(\vec{x} t, \overrightarrow{0} t_{0}\right)\left(\bar{\gamma}_{5} \bar{\gamma}_{n^{\prime}}\right)_{\beta^{\prime \prime} \alpha^{\prime \prime \prime}}\right.} \\
\left.-G_{a \mu^{\prime \prime}, a^{\prime} \nu^{\prime}}^{*}\left(\vec{x} t, \overrightarrow{0} t_{0}\right) G_{b^{\prime} \alpha^{\prime}, b^{\prime} \beta^{\prime}}\left(\overrightarrow{0} t_{0}, \overrightarrow{0} t_{0}\right)\left(\bar{\gamma}_{5} \bar{\gamma}_{n^{\prime}}\right)_{\beta^{\prime} \alpha^{\prime}}\right] \\
\gamma_{m_{\mu^{\prime \prime} \nu^{\prime}}}\left(F_{m n}^{a b}(\vec{x} t)-F_{m n}^{+a b}(\vec{x} t)\right)>.
\end{gathered}
$$


For the $<O_{h}(t) O_{h}^{\dagger}\left(t_{0}\right)>$ matrix element, using eq. (3.29) for $O_{h}(t)$ and following the above procedure, we arrive at (see Fig. 3)

$$
\begin{gathered}
<O_{h}(t) O_{h}^{\dagger}\left(t_{0}\right)>= \\
<\sum_{\vec{x}}-G_{a \mu, c \lambda}\left(\vec{x} t, \overrightarrow{0} t_{0}\right) G_{b \nu, l \sigma}^{*}\left(\vec{x} t, \overrightarrow{0} t_{0}\right) \\
\left(\gamma_{5} \bar{\gamma}_{m}\right)_{\nu \mu}\left(\gamma_{m^{\prime}} \bar{\gamma}_{5}\right)_{\lambda \sigma} \\
\left(F_{m n}^{a b}(\vec{x} t)-F_{m n}^{\dagger a b}(\vec{x} t)\right)\left(F_{m^{\prime} n^{\prime}}^{c d}\left(\overrightarrow{0} t_{0}\right)-F_{m^{\prime} n^{\prime}}^{\dagger c d}\left(\overrightarrow{0} t_{0}\right)\right)^{\dagger}>
\end{gathered}
$$



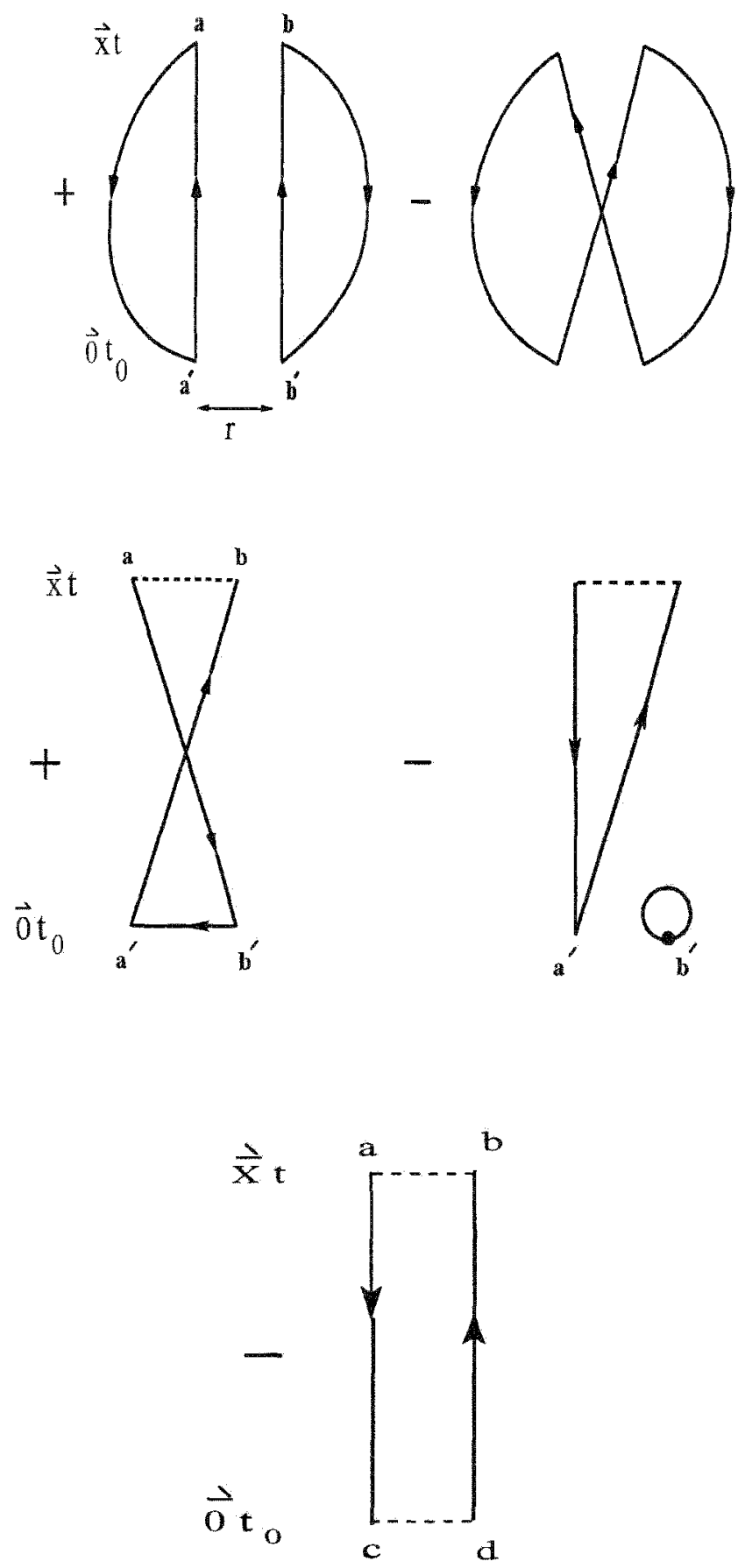

Figure 3: Diagramatic illustrations of the matrix elements $\left\langle O_{\pi a_{1}}(t) O_{\pi a_{1}}^{\dagger}\left(t_{0}\right)\right\rangle$ (upper diagram, eq. (3.56)), $\left\langle O_{h}(t) O_{\pi a_{1}}^{\dagger}\left(t_{0}\right)\right\rangle$ (middle diagram, eq. (3.64)), and $<O_{h}(t) O_{h}^{\dagger}\left(t_{0}\right)>$ (lower diagram, eq. (3.65)). Solid lines represent quark and antiquark propagators $G$ and $G^{*}$ respectively, and the dashed line represents the valence gluon field $F-F^{\dagger}$. Closed loops indicate color index contractions. 


\subsection{Smearing of fields}

The hadron operators used in LQCD simulations create and annihilate linear combinations

$$
\left|\Phi>=\sum_{n>0}\right| n>A_{n}
$$

of the physical states $|n\rangle$ which are ultimately determined by the lattice action. If we are interested in the properties (e.g. mass) of a particular state, say $\left|n_{1}\right\rangle$, it is of great advantage to employ an operator, say $\mathcal{O}_{1}^{\dagger}$, that creates a state $\mid \Psi>$ such that the amplitude $A_{1}$ dominates the linear combination eq. (3.66). The advantage is numerical: The resulting time correlation function will then give rise to a "wide" plateau $m_{\mathrm{eff}}(t)$, see eq. (2.38), and thus greatly simplify the extraction and precision of the mass $m_{1}$ of the state.

A physical baryon state will have a spatial extent of typically about 1 fermi. The operators constructed so far, however, are local or point like. They should not be expected to couple strongly to a spatially extended state. To remedy this situation a process called smearing [68] or fuzzing [69] is used. Smearing and fuzzing both have the effect of producing operators that couple more strongly with lower lying energy states and not with higher lying contaminating states.

A smeared fermion field $\tilde{\psi}(x)$ is constucted by spreading the original field $\psi(x)$ over neighboring sites via [68],

$$
\begin{aligned}
\tilde{\psi}^{0}(x) & =\psi(x) \\
\tilde{\psi}^{k+1}(x) & =\psi^{k}(x)+\sum_{m=1}^{3} \alpha_{m} U_{m}(x) \psi^{k}(x+\hat{m}) \\
& +\sum_{m=1}^{3} \alpha_{m} U_{m}^{\dagger}(x-\hat{m}) \psi^{k}(x-\hat{m})
\end{aligned}
$$


and,

$$
\begin{aligned}
\tilde{\bar{\psi}}^{0}(x) & =\psi(x) \\
\tilde{\bar{\psi}}^{k+1}(x) & =\bar{\psi}^{k}(x)+\sum_{m=1}^{3} \alpha_{m} \bar{\psi}^{k}(x+\hat{m}) U_{m}^{\dagger}(x) \\
& +\sum_{m=1}^{3} \alpha_{m} \bar{\psi}^{k}(x-\hat{m}) U_{m}(x-\hat{m})
\end{aligned}
$$

where $k=0,1,2 \ldots K-1$. Here $\alpha_{m}$ is a strength factor which controls the amount of the smearing and the index $m$ stands for any one of three spatial directions. There is no smearing in the time direction. Note that the link matrices $U(x)$ act as parallel transporters, hence the behavior of $\psi(x), \tilde{\psi}(x)$ and $\bar{\psi}(x), \tilde{\bar{\psi}}(x)$ under gauge transformations is exactly the same.

Smearing is a recursive process that is done $K$ times to form operators used in this analysis. Keeping all smearing levels expands the size of the correlation matrix by a factor $K$. For large $K$ and/or large strength factors $\alpha_{m}$ normalization is useful to keep the magnitude of the correlation functions under control numerically.

To motivate a suitable normalization factor let us, for the duration of this argument, assume that $\psi, \bar{\psi}$ are ordinary Hilbert space operators obeying the anticommutation relation

$$
\left\{\psi_{a \mu}(x), \bar{\psi}_{b \nu}(y)\right\}=\delta_{a b} \delta_{\mu \nu} \delta_{x y}
$$

The smeared fields $\psi^{k}, \bar{\psi}^{k}$ will not satisfy eq. (3.70) because the locality $\propto \delta_{x y}$ is lost in the process. However, after one iteration and at equal sites $x=y$, it is straight forward to show from eqs. (3.68), (3.69), and (3.70) that

$$
\left\{\psi_{a \mu}^{1}(x), \bar{\psi}_{b \nu}^{1}(x)\right\}=\delta_{a b} \delta_{\mu \nu}\left(1+2 \sum_{m=1}^{3} \alpha_{m}^{2}\right) .
$$


We take this to be an indicator of the magnitude of the change in normalization. Thus, after each smearing iteration (3.68) and (3.69) we rescale the fields

$$
\psi^{k+1} \rightarrow N^{-1} \psi^{k+1} \quad, \quad \bar{\psi}^{k+1} \rightarrow N^{-1} \bar{\psi}^{k+1},
$$

where

$$
N=\left(1+2 \sum_{m=1}^{3} \alpha_{m}^{2}\right)^{\frac{1}{2}} .
$$

This procedure is clearly gauge invariant.

The accumulated effect of normalization can be significant in practice. For example, for typical values $\alpha_{m}=2.5, K=3$ and a two-meson operator, the resulting correlator matrix element would acquire a factor $N^{3 \cdot 8} \approx 10^{19}$ if rescaling was not done.

For the same reasons stated at the beginning of this section it is also beneficial to spatially spread out gluon fields with a similar iterative process which is referred to as fuzzing. The fuzzing algorithm used in this analysis is called APE fuzzing [69]. Here a spatial link matrix is replaced by itself plus a real weight $\rho_{n}$ times the sum of its four neigbhboring staples in the spatial planes.

$$
\begin{aligned}
U_{m}^{0}(x) & =U_{m}(x) \\
U_{m}^{k+1}(x) & =U_{m}^{k}(x)+\sum_{\substack{n=1 \\
n \neq m}}^{3} \rho_{n}\left[U_{n}^{k}(x) U_{m}^{k}(x+\hat{n}) U_{n}^{k^{\dagger}}(x+\hat{m})\right. \\
& \left.+U_{n}^{k^{\dagger}}(x-\hat{n}) U_{m}^{k}(x-\hat{n}) U_{n}^{k}(x+\hat{m}-\hat{n})\right]
\end{aligned}
$$

for $m=1,2,3$. The iterated link variables clearly transform covariantly under gauge transformations, however, they are no longer elements of $\mathrm{SU}(3)$. They may be projected back into $\mathrm{SU}(3)$ by various methods [69]. We here adopt a simple 
normalization prescription with no projection back into $\mathrm{SU}(3)$. For reasons similar to those discussed above in the context of quark fields, after each iteration step eq. (3.75), we rescale the fuzzy link variables as

$$
U_{m}^{k+1}(x) \rightarrow N^{-1} U_{m}^{k+1}(x) \quad \text { where } \quad N=\left(\frac{1}{3} \operatorname{Tr} U_{m}^{k+1^{\dagger}}(x) U_{m}^{k+1}(x)\right)^{\frac{1}{2}}
$$

This procedure is clearly gauge invariant.

Finally, fuzzy link variables may be used in the smearing of fermion fields, thus replacing $U_{m}(x) \rightarrow U_{m}^{k}(x)$ in eq's (3.68) and (3.69). 


\section{Simulation and analysis}

\subsection{Goal of this simulation.}

The goal of this numerical simulation is to produce energy or mass spectra from the set of operators established in the previous section and to use these spectra to extract scattering phase shifts via Lüscher's method. This requires, first, finding the eigenvalues of a correlation matrix, and second, processing their time dependence through effective mass functions, for example, to get the energy levels.

\subsection{Lattice parameters.}

All lattice simulations were performed using the Wilson plaquette action eq. (2.19) and Wilson fermions eq. (2.24) in the quenched approximation. The lattice sizes used were $6^{3} \times 24,8^{3} \times 24,10^{3} \times 24$, and $12^{3} \times 24$. The number of configurations for these lattice sizes were $200,200,180$, and 160 respectively. A heat bath algorithm $[70,71]$ was employed to generate the configurations. All simulations were done at $\beta=6.15$ with bare anisotropy $\xi=a_{s} / a_{t}=2$. Fermion matrix inversions were done at four values of the hopping parameter $\kappa=.1400, .1360, .1320$, and .1280 using a multiple mass inverter [72]. For these simulations, the sources were always placed in the spatial middle of the lattice and at timeslice $t_{0}=3$. 
As a matter of course, the following single-meson operators,

$$
\begin{aligned}
O_{\pi}(t) & =\sum_{\vec{x}} \bar{d}(\vec{x}, t) \gamma_{5} u(\vec{x}, t) \\
O_{\rho}(t) & =\sum_{\vec{x}} \bar{d}(\vec{x}, t) \gamma_{i} u(\vec{x}, t) \\
O_{a_{1}}(t) & =\sum_{\vec{x}} \bar{d}(\vec{x}, t) \gamma_{5} \gamma_{i} u(\vec{x}, t)
\end{aligned}
$$

and the hybrid operator $O_{h}(t)$, as given by eq. (3.29), were employed.

Using three smearing levels, the corresponding $3 \times 3$ correlation matrices are then diagonalized. Their eigenvalues give rise to effective mass functions from which the ground state masses of the four mesons can be extracted as described in Section 2. If the correlation function is dominated by a single state, then a plot of these effective masses reveals a plateau through which a horizontal line is fit using a least squares technique. The result of such a fit is a dimensionless number for $a_{t} m$, where $a_{t}$ is the lattice constant in the time direction and $m$ is the physical mass of the meson. For example, Fig. 4 shows a correlation function for the $a_{1}$ meson and the resulting effective mass function.

Results obtained in this way for the $\pi, \rho, a_{1}$ and $h$ mesons using operator eqs. (4.1-.3), and (3.29) with two levels of smearing are shown in Table 1. These numbers will be needed to extrapolate later results to $m_{\pi} \rightarrow 0$ and to set the physical scale, see Section 4.3. 

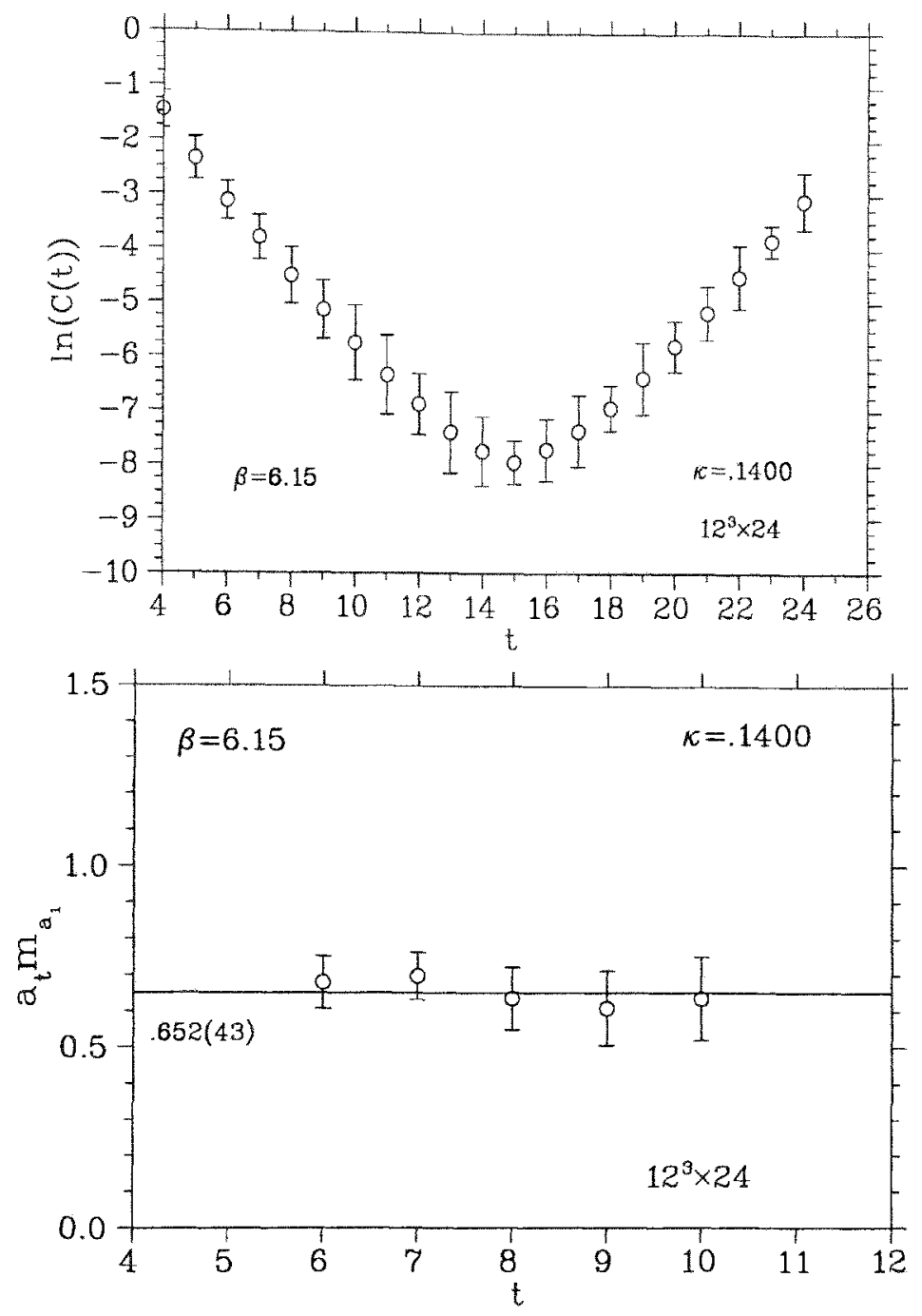

Figure 4: Correlation function(upper panel) for the $a_{1}$ meson with two levels of smearing on the $12^{3} \times 24$ lattice, and the resulting effective mass plot(lower panel) at $\kappa=.1400$. Error bars represent statistical errors.

Errors presented in this work are statistical errors using the jackknife technique [73], unless otherwise stated.

The mass of the hybrid meson is much harder to obtain because the gauge links contained in the operator $O_{h}$, see eq. (3.29), give rise to considerable noise in the numerical signal. In order to address this problem, observe that the gauge 


\begin{tabular}{|c|c|c|c|c|}
\hline$\kappa$ & $a_{t} m_{\pi}$ & $a_{t} m_{\rho}$ & $a_{t} m_{a_{1}}$ & $a_{t} m_{h}$ \\
\hline .1400 & $.533(41)$ & $.550(31)$ & $.652(43)$ & $.631(253)$ \\
.1360 & $.644(34)$ & $.646(29)$ & $.752(31)$ & $.753(214)$ \\
.1320 & $.748(31)$ & $.751(32)$ & $.857(26)$ & $.850(261)$ \\
.1280 & $.849(35)$ & $.852(27)$ & $.964(32)$ & $.948(237)$ \\
\hline
\end{tabular}

\begin{tabular}{|c|c|c|c|c|}
\hline$\kappa$ & $a_{t} m_{\pi}$ & $a_{t} m_{\rho}$ & $a_{t} m_{a_{1}}$ & $a_{t} m_{h}$ \\
\hline .1400 & $.540(45)$ & $.541(33)$ & $.639(41)$ & $.618(241)$ \\
.1360 & $.649(33)$ & $.623(27)$ & $.745(33)$ & $.714(231)$ \\
.1320 & $.742(26)$ & $.728(31)$ & $.853(36)$ & $.807(246)$ \\
.1280 & $.853(40)$ & $.834(30)$ & $.961(29)$ & $.914(215)$ \\
\hline
\end{tabular}

Table 1: Values of the hopping parameter $\kappa$ and the resulting $\pi, \rho, a_{1}$ and $h$ masses, in dimensionless units for the $12^{3} \times 24$ lattice(upper table) and the $10^{3} \times 24$ lattice(lower table). Errors are statistical.

field action $S_{g}[U]$ is invariant with respect to replacing $U$ with $U^{*}$. Hence, for each configuration $[U]$ the complex conjugate configuration $\left[U^{*}\right]$ is equally probable. It is thus admissible to enforce this symmetry. That is, once propagators are computed for an equilibrium field $[U]$, the complex conjugate of the same field, $\left[U^{*}\right]$, is then used to compute propagators, and this is counted as an additional configuration leading to $400,400,360,320$ propagators for the four lattices. This helps enforce charge conjugation parity of the operators and it reduced the noise coming from the hybrid operator. 
In Fig. 5 a correlation function for the hybrid operator eq. (3.29) is shown. Comparison to Fig. 4 reveals that the hybrid signal still is significantly noisier. Accordingly the quality of the resulting effective mass function, see Fig. 5, is also diminished. These observations hold true, m.m., for the full simulation including the two-body decay channel.
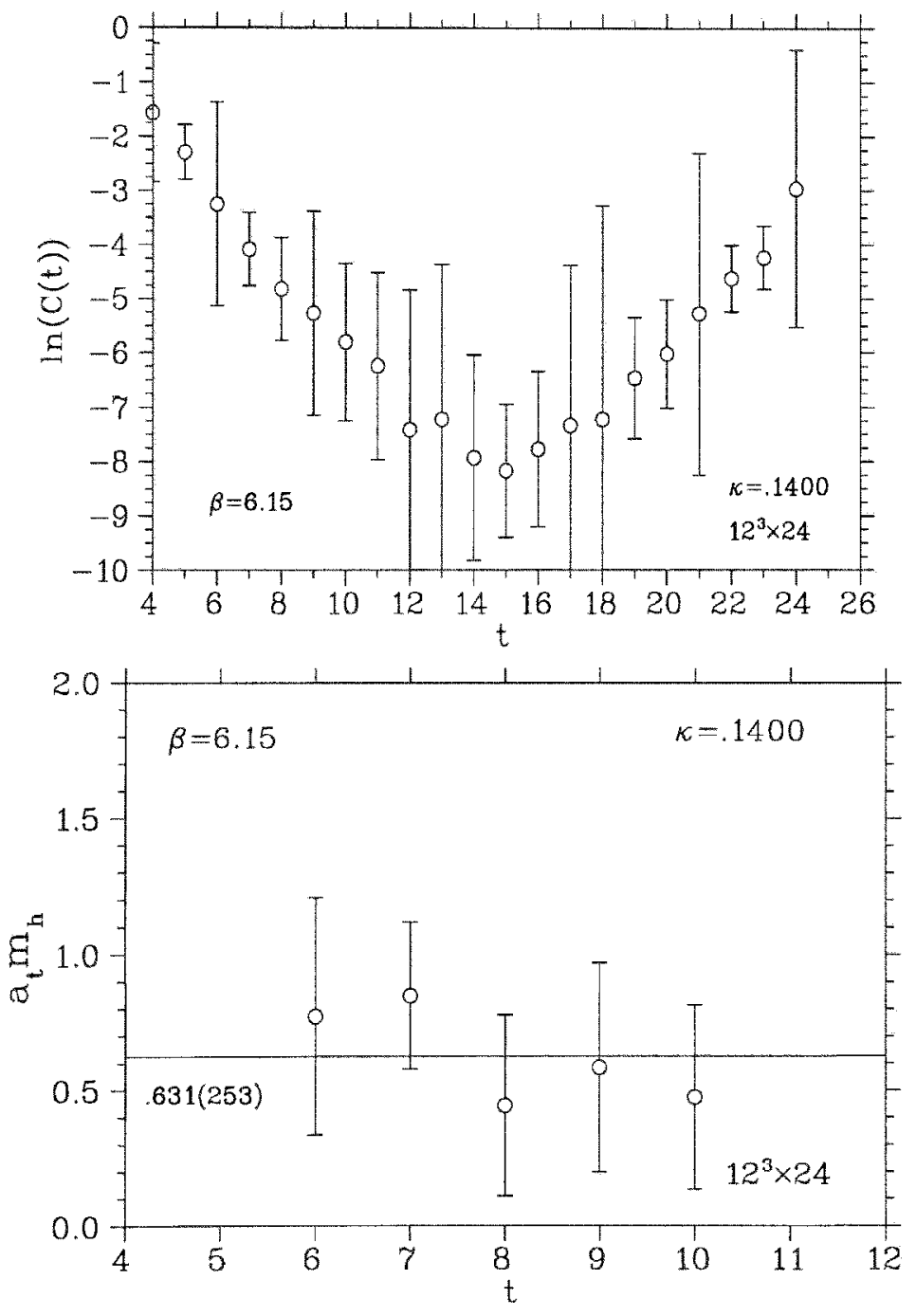

Figure 5: Exotic hybrid correlation function (upper panel) for 2 levels of smearing on the $12^{3} \times 24$ lattice, and the resulting effective mass plot (lower panel) at $\kappa=.1400$. 


\subsection{Extrapolation to the physical mass region, and setting the physical scale.}

Meson masses were obtained at four values of the Wilson hopping parameter $\kappa$ (see Table 1). It is useful to study the dependence of the computed $\rho, a_{1}$ and $h$ masses on the pion mass. Predictions for this dependence come from chiral perturbation theory [74]. For example, the vector meson masses $M_{\rho}=a_{t} m_{\rho}$ can be fitted to a quadratic polynomial in the square of the pion mass $M_{\pi}=a_{t} m_{\pi}[75]$,

$$
M_{\rho} \approx A+B M_{\pi}^{2}+C M_{\pi}^{4}
$$

This model, if applicable, allows extrapolation of $M_{\rho}$ to the physical pion mass region, i.e. small $M_{\pi}$. A logarithmic term can also be added [76]

$$
M_{\rho} \approx A+B M_{\pi}^{2}+C M_{\pi}^{4}+\ln \left(M_{\pi}^{2}\right)
$$

provided the model is applied in the asymptotic region, i.e. large $M_{\pi}$.

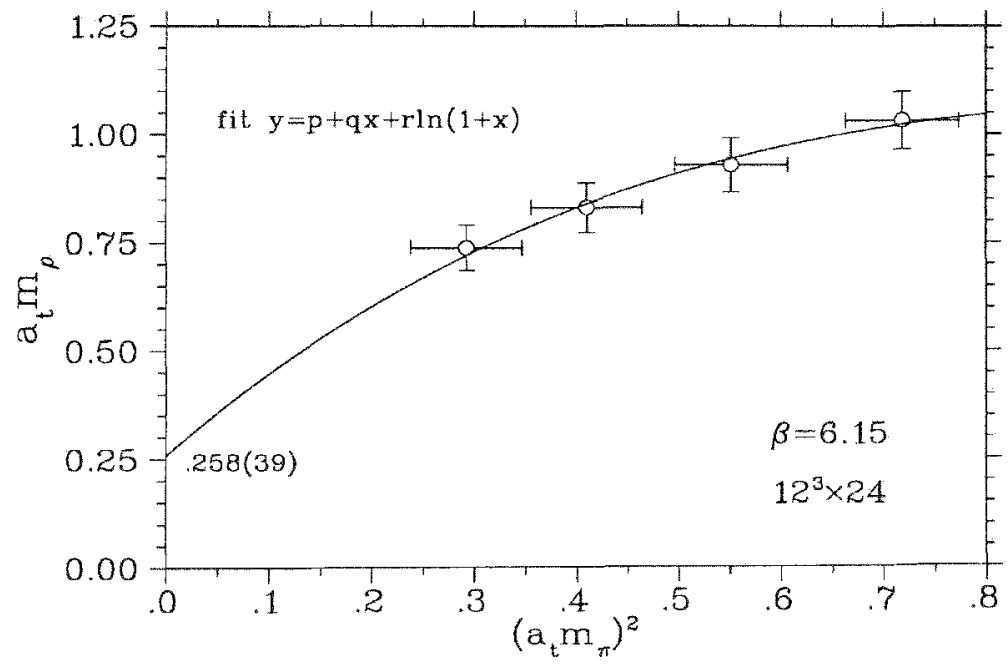

Figure 6: Extrapolation for the $\rho$ meson mass using the $12^{3} \times 24$ lattice. 
For the combined $h$ and $\pi a_{1}$ system no predictions for the dependence of the spectral masses $W$ on $m_{\pi}$ are currently available. We will adopt the three parameter fit model

$$
y=p+q x+r \ln (1+x) \quad \text { with } x=\left(a_{t} m_{\pi}\right)^{2}
$$

and $y$ being a meson mass $a_{t} m$, or a spectral mass $a_{t} W$ as the case may be. While inspired by eqs. (4.4) and (4.5) the model is purely heuristic. The main reason for it's use is that it yields $\chi^{2}$-fits to our data that are consistently superior to those obtainable using eqs. (4.4) or (4.5). Examples of such fits are shown in Fig. 6 for the $p$ meson mass, and in Fig. 7 for the $a_{1}$ and hybrid masses. From those fits extrapolations to the physical pion mass are possible. Results are shown in Table 2. These can be used to set the physical mass or length scale for the simulation.
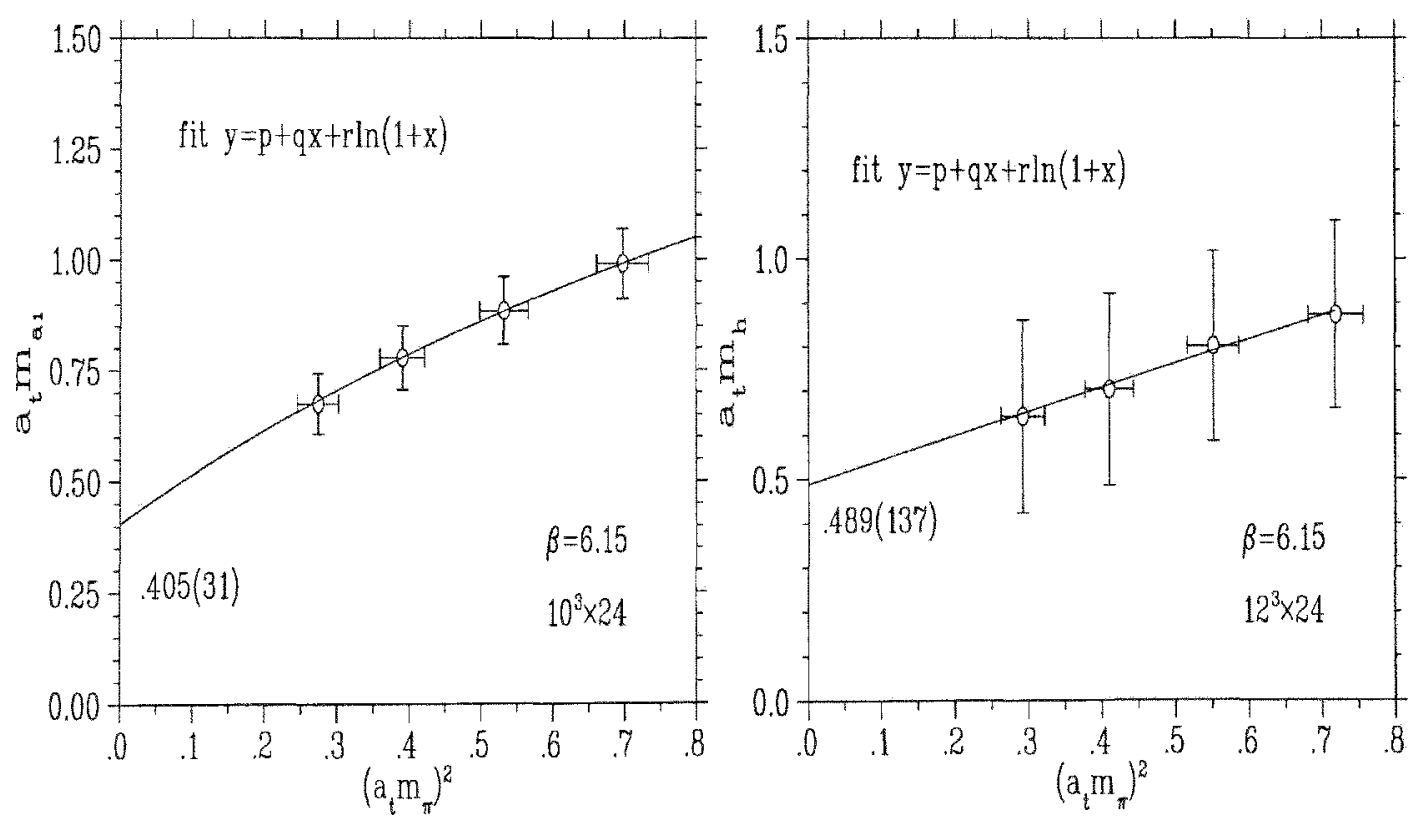

Figure 7: Extrapolation for the $a_{1}$ meson mass(left panel) using the $10^{3} \times 24$ lattice and $h$ meson mass(right panel) using the $12^{3} \times 24$ lattice. 
To set the physical scale, an experimental mass, say $m$, has to be used as input. Then $a_{t} m=p$, where $p$ is taken from fits with eq. (4.6), yields the lattice constant in physical units $a_{t}=p / m$. If the $\rho$ meson mass is used we obtain ${ }^{1}$

$$
a_{t}=0.332(50) \mathrm{GeV}^{-1}=0.066(10) \mathrm{fm} \quad\left[m_{\rho}=776 \mathrm{MeV}\right]
$$

If the $a_{1}$ meson sets the scale then

$$
a_{t}=0.298(28) \mathrm{GeV}^{-1}=0.059(06) \mathrm{fm} \quad\left[m_{a_{1}}=1230 \mathrm{MeV}\right]
$$

The above numbers are based on the $12^{3} \times 24$ lattice. Unless otherwise indicated the $\rho$-meson based scale (4.7) on this lattice will be used to present dimensional values.

\begin{tabular}{|c|c|c|c|}
\hline$L$ & $a_{t} m_{\rho}$ & $a_{t} m_{a_{1}}$ & $a_{t} m_{h}$ \\
\hline 12 & $.258(39)$ & $.438(26)$ & $.489(137)$ \\
10 & $.260(34)$ & $.405(31)$ & $.467(143)$ \\
\hline
\end{tabular}

Table 2: Extrapolated masses for the $\rho, a_{1}$ and $h$ mesons on lattices $L^{3} \times 24$.

\footnotetext{
${ }^{1}$ Units are such that $\hbar=c=1$. The conversion factor between $\mathrm{GeV}$ and fm is given by 0.1973 $\mathrm{GeV} \cdot \mathrm{fm}=1$.
} 
Using the $\rho$-scale the mass of the $a_{1}$, when extrapolated to zero pion mass, is $1.21(18) \mathrm{GeV}$ on the $10^{3} \times 24$ lattice and $1.31(11) \mathrm{GeV}$ on the $12^{3} \times 24$ lattice. These values agree within errors with the $1.23 \mathrm{GeV}$ mass listed by the Particle Data Group [3]. On the $10^{3} \times 24$ lattice, the mass of the exotic is $1.46(31) \mathrm{GeV}$ using the $\rho$ meson to set the scale and $1.73(39) \mathrm{GeV}$ using the $a_{1}$ to set the scale. More plots pertaining to the exotic meson are contained in Appendix $H$. The value of $\sim 1.5$ $\mathrm{GeV}$ is somewhat lower than previous lattice calculations, which ranged from $\sim 1.7$ to $2.1 \mathrm{GeV}[17]$, but it is in good agreement with experimental observations of these exotic resonances, which range from $\sim 1.37$ to $1.40 \mathrm{GeV}[17,77,78]$. Note that to calculate the hybrid mass, three effective mass functions have to be evaluated one for the hybrid, one for the rho, and one for the pion masses. Due to variability in selecting the points used for effective mass functions, there is going to be some systematic error in the outcome depending on which points are selected. This is one possible reason why the hybrid mass is lower in this calculation than in others. Also, to keep computer processing times reasonable, the values of the pion mass used for this calculation are high, ranging from $1.5 \mathrm{GeV}$ to $2.4 \mathrm{GeV}$. This is another source of variation between these results and previous lattice results. 


\subsection{Projecting vs. diagonalizing at all $\mathrm{t}$}

A standard analysis method for extracting time correlation functions from an $N \times N$ matrix $C\left(t, t_{0}\right)$ is based on solving the generalized eigenvalue problem [52]

$$
C\left(t, t_{0}\right) \Psi(t)=C\left(t_{1}, t_{0}\right) \Psi(t) \Lambda(t)
$$

where $t_{1}$ is fixed, $\Psi(t)$ is an $N \times N$ matrix, its columns being the generalized eigenvectors, and $\Lambda(t)$ is diagonal. By construction, $C\left(t_{1}, t_{0}\right)$ is Hermitian, thus having real eigenvalues. We further require that $C\left(t_{1}, t_{0}\right)$ be positive definite. To ensure the latter, $t_{1}$ should be an "early" time slice. Here we use $t_{1}-t_{0}=4$. The generalized eigenvalue problem, eq. (4.9), can then be cast into an ordinary one by first diagonalizing

$$
C\left(t_{1}, t_{0}\right)=V\left(t_{1}, t_{0}\right) D\left(t_{1}, t_{0}\right) V^{\dagger}\left(t_{1}, t_{0}\right)
$$

where $V\left(t_{1}, t_{0}\right)$ is now unitary and $D\left(t_{1}, t_{0}\right)$ is real diagonal and positive definite. Inserting eq. (4.10) into eq. (4.9) we are lead to define

$$
\hat{C}(t)=\frac{1}{\sqrt{D\left(t_{1}, t_{0}\right)}} V^{\dagger}\left(t_{1}, t_{0}\right) C\left(t, t_{0}\right) V\left(t_{1}, t_{0}\right) \frac{1}{\sqrt{D\left(t_{1}, t_{0}\right)}},
$$

and

$$
\hat{\Psi}(t)=\sqrt{D\left(t_{1}, t_{0}\right)} V^{\dagger}\left(t_{1}, t_{0}\right) \Psi(t),
$$

in terms of which eq. (4.9) assumes the form of an ordinary eigenvalue problem

$$
\hat{C}(t) \hat{\Psi}(t)=\hat{\Psi}(t) \Lambda(t)
$$

where $\hat{\Psi}(t)$ now is unitary and where $\Lambda(t)=\operatorname{diag}\left(\lambda_{1}(t) \ldots \lambda_{N}(t)\right)$ contains the eigenvalues of the generalized problem, eq. (4.9). Constructing $\hat{C}$ according to eq. (4.11) merely amounts to a linear transformation of the set of operators $\mathcal{O}_{X}, X=1 \ldots N$, that define the correlation matrix. From eqs. (3.32) and (4.10) it is obvious that

$$
\hat{C}_{X Y}(t)=<\hat{O}_{X}(t) \hat{O}_{Y}^{\dagger}\left(t_{0}\right)>-<\hat{O}_{X}(t)><\hat{O}_{Y}^{\dagger}\left(t_{0}\right)>
$$


where

$$
\hat{O}_{X}(t)=\sum_{X^{\prime}=1}^{N}\left(\frac{1}{\sqrt{D\left(t_{1}, t_{0}\right)}} V^{\dagger}\left(t_{1}, t_{0}\right)\right)_{X X^{\prime}} O_{X^{\prime}}(t) \text { etc. }
$$

Because of $\hat{C}\left(t_{1}\right)=\mathbf{1}$, the new set $\hat{O}_{X}(t)$ of operators creates quantum states that are orthogonal and normalized at $t=t_{1}$. Provided that these match the "true" states of the theory, the eigenvectors of $\hat{C}(t)$, i.e. the columns of $\hat{\Psi}(t)$, will stay orthogonal as $t \geq t_{1}$ increases to the extent allowed by the errors of the simulation. Consequently $\Lambda(t)$ will be approximately diagonal for $t \geq t_{1}$. From an analysis point of view there are two options: One may diagonalize $\hat{C}(t)$, eq. (4.11) on each timeslice $t$ seperately (see Appendix A) and thus obtain the eigenvalues $\lambda_{n}(t), n=1 \ldots N$. Alternatively, the diagonal elements of $\hat{C}(t)$ may be taken as an approximation to its eigenvalues,

$$
\lambda_{n}(t) \approx \hat{C}_{n n}(t), n=1 \ldots N
$$

The latter approach has the advantage that statistical fluctuations in the signal for the $\lambda_{n}(t)$ are reduced, as our numerical results show. This is plausible because fluctuations of the eigenvector components are effectively frozen in the second approach. Figure 8 displays an example of correlators using both the basis transformation technique and diagonalizing on each timeslice. As it turns out effective mass function plateaus typically develop in the time interval $6 \lesssim t \lesssim 11$, or so. In this region the projection technique yields more stable correlation functions, particularly for the excited states. For this reason, we will continue our analysis with the projected correlators and simply refer to $\hat{C}_{n n}(t)$ as eigenvalues $\lambda_{n}(t)$ for simplicity. In this sense, the eigenvalues $\lambda_{n}(t), n=1 \ldots 6$, then give rise to effective mass functions from which the spectral energies $W_{n}$ are obtained. An example for the $12^{3} \times 24$ lattice at $\kappa=.1400$ is shown in Fig. 9. 
All correlator values are normalized to timeslice $t=5$. No points with timeslice $t<5$ were used for mass calculations. Figure 10 shows the spectral energies $W_{n}, n=$ $1 \ldots 6$, from the $12^{3} \times 24$ and $10^{3} \times 24$ lattices as they depend on the squared pion mass, $x=\left(a_{t} m_{\pi}\right)^{2}$ along with fits using the model eq. (4.5). A list of the numerical results for two lattice sizes are in Table 3. These excited spectra, including the extrapolations to physical pion mass $m_{\pi} \rightarrow 0$ provide the input to Lüscher's method for calculating scattering phase shifts, and ultimately, decay widths. 

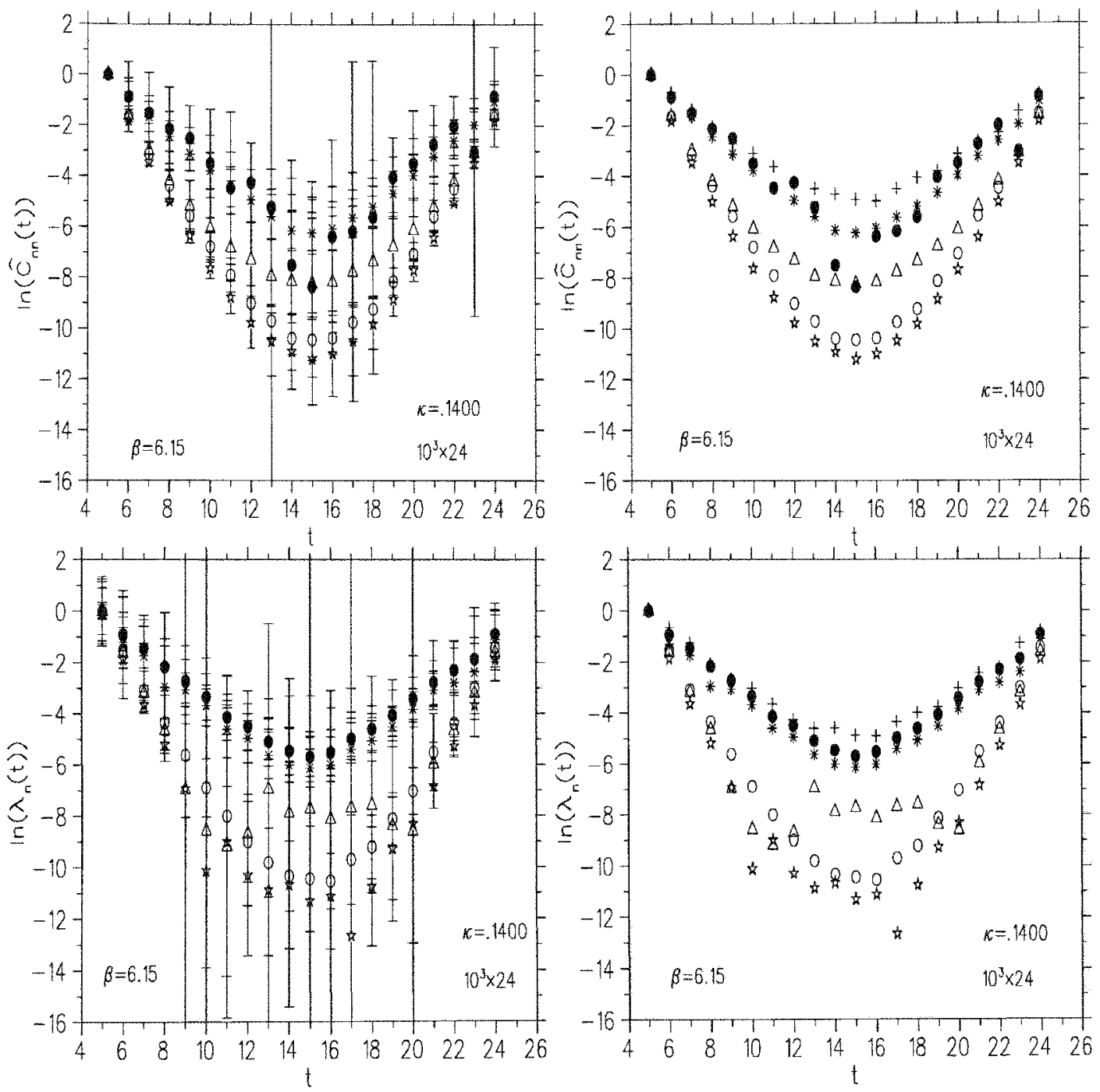

Figure 8: Comparison of the eigenvalues of the $6 \times 6$ correlation matrix $\hat{C}(t)$ for the $10^{3} \times 24$ lattice using the two analysis techniques described in Section 4.4. Results for "diagonalization of each timeslice" (lower panels) versus "diagonal" elements of the correlator $\hat{C}(t)$ projected at $t_{1}=5$ (upper panels). Shown with and without error bars for clarity. 

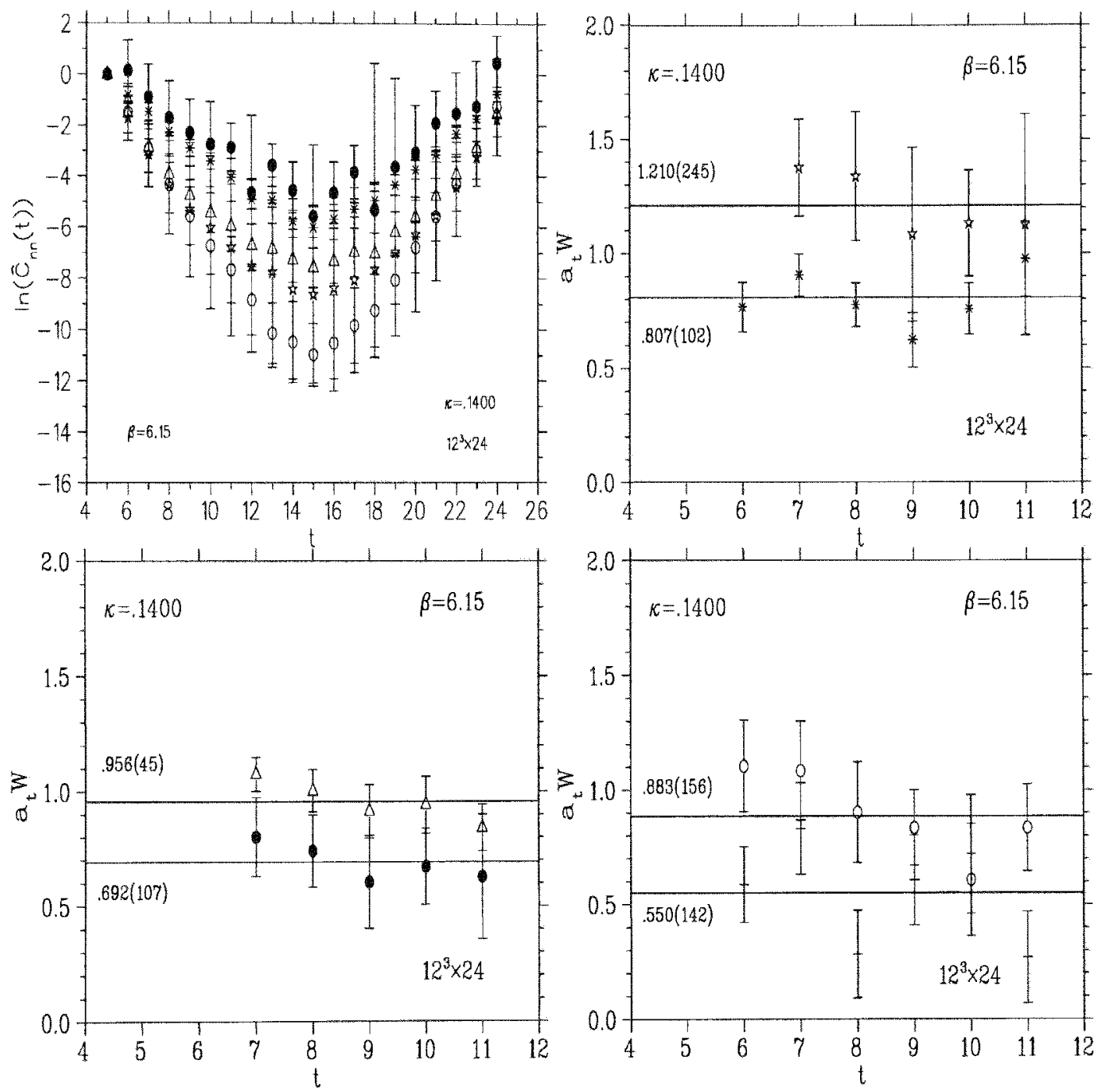

Figure 9: Eigenvalues(upper left) of the $6 \times 6$ correlation matrix along with effective masses of all six eigenvalues for the $12^{3} \times 24$ lattice. Error bars represent statistical errors. 

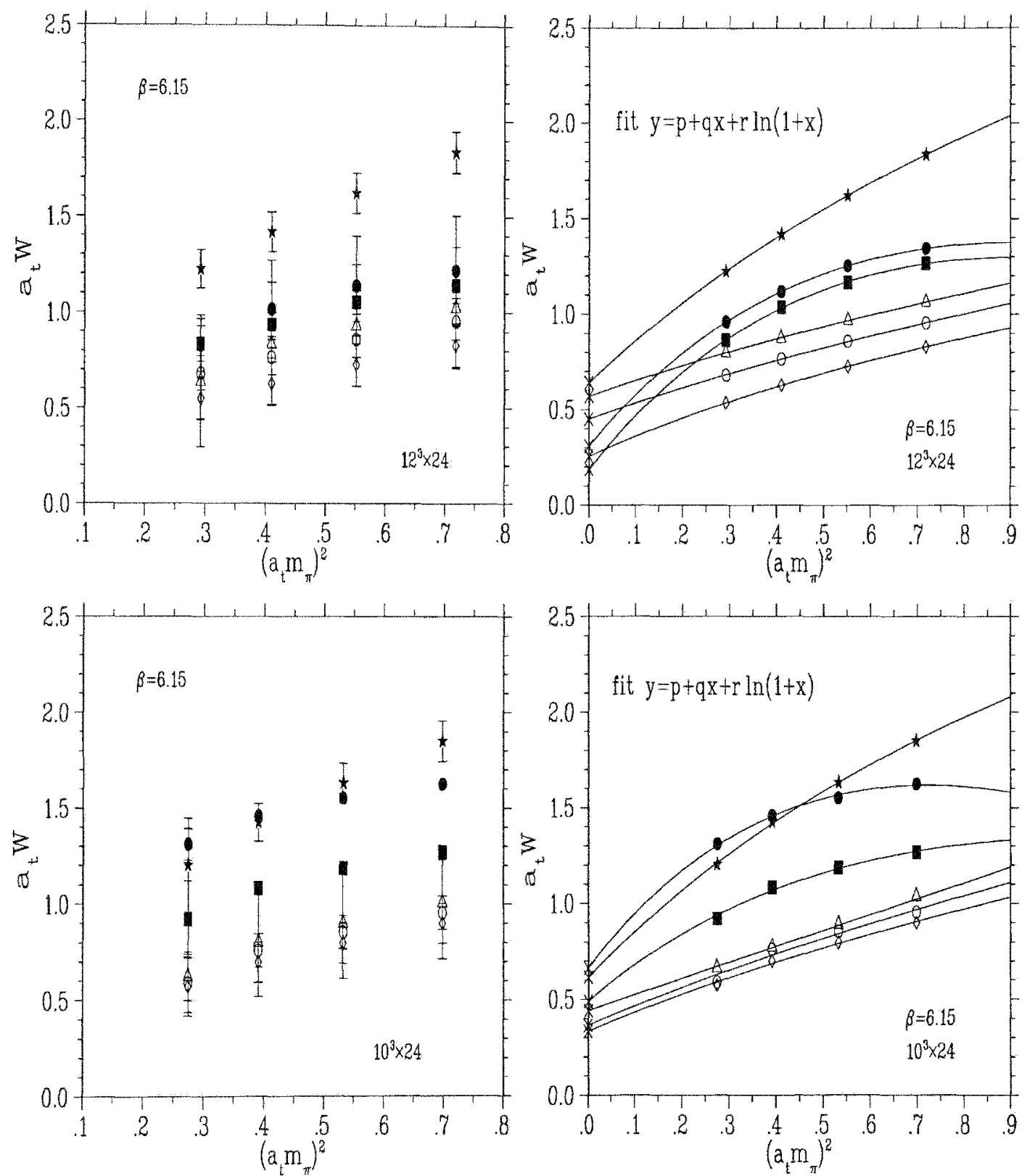

Figure 10: Energy spectra for the $12^{3} \times 24$ lattice (upper panels) and for the $10^{3} \times 24$ lattice (lower panels) extrapolated to $m_{\pi} \rightarrow 0$. Error bars omitted on the right panels for clarity. 


\begin{tabular}{|c|c|c|c|c|c|c|c|}
\hline$\kappa$ & $a_{t} m_{\pi}$ & $a_{t} W_{1}$ & $a_{t} W_{2}$ & $a_{t} W_{3}$ & $a_{t} W_{4}$ & $a_{t} W_{5}$ & $a_{t} W_{6}$ \\
\hline .1400 & $.540(45)$ & $.550(142)$ & $.692(107)$ & $.807(102)$ & $.883(126)$ & $1.210(85)$ & $.956(45)$ \\
.1360 & $.649(33)$ & $.691(21)$ & $.787(34)$ & $.807(35)$ & $1.030(113)$ & $1.134(27)$ & $1.420(43)$ \\
.1320 & $.742(26)$ & $.741(38)$ & $.893(41)$ & $.908(42)$ & $1.180(102)$ & $1.253(31)$ & $1.616(28)$ \\
.1280 & $.853(40)$ & $.821(102)$ & $.927(85)$ & $1.034(73)$ & $1.261(53)$ & $1.312(43)$ & $1.849(41)$ \\
\hline
\end{tabular}

\begin{tabular}{|c|c|c|c|c|c|c|c|}
\hline$\kappa$ & $a_{t} m_{\pi}$ & $a_{t} W_{1}$ & $a_{t} W_{2}$ & $a_{t} W_{3}$ & $a_{t} W_{4}$ & $a_{t} W_{5}$ & $a_{t} W_{6}$ \\
\hline .1400 & $.533(41)$ & $.573(72)$ & $.592(113)$ & $.614(57)$ & $.910(112)$ & $1.202(94)$ & $1.311(58)$ \\
.1360 & $.644(34)$ & $.693(105)$ & $.705(48)$ & $.738(53)$ & $1.120(76)$ & $1.417(1033)$ & $1.428(59)$ \\
.1320 & $.748(31)$ & $.751(112)$ & $.783(42)$ & $.804(39)$ & $1.140(127)$ & $1.640(92)$ & $1.553(86)$ \\
.1280 & $.849(35)$ & $.784(61)$ & $.811(51)$ & $.907(51)$ & $1.240(85)$ & $1.783(91)$ & $1.621(126)$ \\
\hline
\end{tabular}

Table 3: Energy spectra $W_{n}, n=1 \ldots 6$ from the $12^{3} \times 24$ lattice (upper table) and the $10^{3} \times 24$ lattice (lower table) at four pion masses. 


\section{Lüscher's method for scattering phase shifts}

In a series of seminal papers $[49,51,52]$ Lüscher has shown how to calculate continuum scattering phase shifts from an LQCD simulation in Euclidean space in a finite volume. We here present an introduction to this method.

\subsection{Basic idea behind Lüscher's formulae.}

Lüscher related continuum scattering phase shifts to the discrete two-body energy spectrum in a finite periodic box by matching poles of interacting Green functions (propagators) that live on the finite-sized periodic lattice to the poles of free Green functions in infinite volume [51] . Because the lattice Green functions comprise the interaction, comparison in the asymptotic (free) region yields the phase shifts.

To illustrate, in one space dimension, the central idea behind this [52] consider a solution $\psi(x)=e^{i k x}$ of a Schrödinger equation with momentum $k$ in the noninteracting case. Choosing $k L=2 \pi n$ the solution is periodic, satisfying $\psi(x)=$ $\psi(x+L)$, see Fig. 11(a) for $k=k_{0}$ and $n=4$. If an attractive potential with range $|x| \leq 0.2 L$ is turned on, the periodic boundary condition would be violated, see Fig. 11(b), unless the kinematical phase $k L$ changes. This change in the phase $k L$, made to maintain original periodicity, is therefore a measure of the phase shift $\delta$, see Fig. 11(c). For the plane wave, $\psi(x)=e^{i k x}$, the relation between the phase shift and wave number at the boundaries is, 


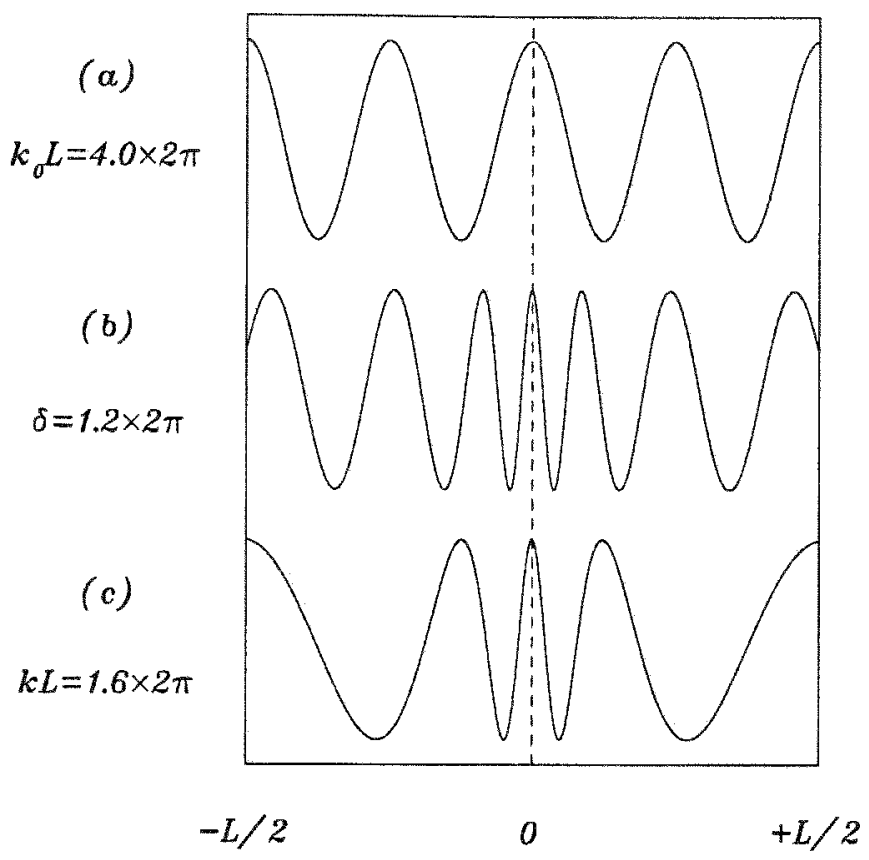

Figure 11: Generic solutions $\psi(x)$ of a Schrödinger equation: (a) no interaction, (b) an attractive potential around $x \approx 0$, and (c) with decreased wave number $k$ to restore the original phase conditions, from Ref. [79].

$$
e^{i\left(k \frac{L}{2}+\delta\right)}=e^{i\left(k \frac{-L}{2}-\delta\right)} \rightarrow e^{2 i \delta}=e^{-i k L}
$$

The kinematical phase $k L$ is changed by varying $k$ or $L$. Varying $L$ is similar to a finite-size effect. Finding the change in $k$ at fixed $L$ is equivalent to finding all (discrete) two-body levels $W_{n}$ for the interacting case.

This method, which already appears in Ref. [61], was used by Lüscher and Wolff [52] to obtain scattering phase shifts in an $O(3)$ nonlinear sigma model on a $1+1$ dimensional lattice and by Göckeler et al [53] to obtain phase shifts for an $\mathrm{O}(4)$ nonlinear sigma model on a $3+1$ dimensional lattice. 
The derivation of a three dimensional equivalent to eq. (5.1) is obtained starting with the Helmholtz equation,

$$
\left(\nabla^{2}+k^{2}\right) G(\vec{r}, k)=\delta(\vec{r})
$$

where $G(\vec{r}, k)$ is a Green function of the two-particle system having the Fourier representation,

$$
G(\vec{r}, k)=\frac{1}{L^{3}} \sum_{\vec{p} \in \Gamma} \frac{e^{i \vec{p} \cdot \vec{r}}}{\left(\vec{p}^{2}-k^{2}\right)}
$$

where $\Gamma$ is the set of lattice momenta in a periodic box of volume $L^{3}$. This Green function can be written (knowing momentum is quantized on a lattice, $\vec{p}=\frac{2 \pi \vec{n}}{L}$ ) as,

$$
G(\vec{r}, k)=\frac{1}{L^{3}} \sum_{\vec{n} \in L^{3}} \frac{e^{i \frac{2 \pi}{L} \vec{n} \cdot \vec{r}}}{\left(\frac{2 \pi}{L}\right)^{2}\left(n^{2}-q^{2}\right)}, \text { with } q=\frac{k L}{2 \pi} .
$$

Note that $\vec{n}$ is being used as a spatial lattice index and not an energy spectra index. The spherical components defined through

$$
G_{l m}(\vec{r}, k)=\mathcal{Y}_{l m}(\vec{\nabla}) G(\vec{r}, k), \quad \mathcal{Y}_{l m}(\vec{r})=r^{l} Y_{l m}(\theta, \phi)
$$

where $Y_{l m}$ are spherical harmonics, can be written as a product of a generalized $\zeta$-function $\mathcal{Z}_{l m}\left(1 ; q^{2}\right)$, a Bessel function $J_{l}(k r)$, and a factor $f_{a}(k)$,

$$
G_{l m}(\vec{r}, k)=f_{a}(k) \mathcal{Z}_{l m}\left(1 ; q^{2}\right) J_{l}(k r)
$$

Here the generalized $\zeta$-function is defined as,

$$
\mathcal{Z}_{l m}\left(s ; q^{2}\right)=\sum_{n=1}^{\Gamma} \frac{Y_{l m}(\theta, \phi) n^{l}}{\left(n^{2}-q^{2}\right)} .
$$

The wave function for the two-particle state can be written as a superposition of these Green functions times a coefficient $v_{l m}$. However, these Green functions have a singularity at $r=0$ which must be removed by adding a Neumann function $N_{l}(k r)$ times a $k$ dependent coefficient $f_{b}(k)[51]$.

$$
\psi_{l m}(\vec{r}, k)=v_{l m}\left(G_{l m}(\vec{r}, k)+f_{b}(k) N_{l}(k r)\right)
$$


This wave function also has representation in terms of Bessel and Neumann functions,

$$
\psi_{l m}(\vec{r}, k)=b_{l m}\left(\alpha_{l}(k) J_{l}(k r) \pm i \beta_{l}(k) N_{l}(k r)\right)
$$

where the \pm indicates either an incoming $(-)$ or outgoing $(+)$ spherical wavefront. The associated scattering phase is given by,

$$
e^{2 i \delta_{l}(k)}=\frac{\alpha_{l}(k)+i \beta_{l}(k)}{\alpha_{l}(k)-i \beta_{l}(k)}
$$

which can be regarded as the 3 -d equivalent [51] to eq. (5.1). Equating coefficients of $J_{l}$ and $N_{l}$ from eqs. (5.8) and (5.9) leaves

$$
b_{l m} \alpha_{l}(k)=v_{l m} \frac{(-1)^{l}}{4 \pi} k^{l+1} \frac{1}{\pi^{\frac{3}{2}} q} \mathcal{Z}_{l m}\left(1, q^{2}\right)
$$

and,

$$
b_{l m} \beta_{l}(k)=v_{l m} \frac{(-1)^{l}}{4 \pi} k^{l+1} .
$$

Taking a ratio of the modulation factors $\alpha_{l}(k)$ and $\beta_{l}(k)$ gives,

$$
\frac{\alpha_{l}(k)}{\beta_{l}(k)}=\frac{1}{\pi^{\frac{3}{2}} q} \mathcal{Z}_{l m}\left(1, q^{2}\right)
$$

Substituting this ratio into eq. (5.10), after some algebra, one obtains a simple formula for the scattering phase [49]. For partial wave $l=0$ it reads

$$
\tan \delta_{0}=-\frac{\pi^{3 / 2} q}{\mathcal{Z}_{00}\left(1 ; q^{2}\right)} \quad, \quad q=\frac{k L}{2 \pi} .
$$




\subsection{Application of Lüscher's formulae.}

Once the energy eigenvalues $W_{n}$ are obtained from the lattice, the momenta $k_{n}$ of the decay products are found by solving the relativistic dispersion relation for $k_{n}$,

$$
W_{n}=\sqrt{m_{\pi}^{2}+k_{n}^{2}}+\sqrt{m_{a_{1}}^{2}+k_{n}^{2}}
$$

Only energies $W_{n}$ that fall within the elastic region,

$$
\left(m_{\pi}+m_{a_{1}}\right)<W_{n}<2\left(m_{\pi}+m_{a_{1}}\right)
$$

are used.

Recalling that all states are created with zero total momentum, these energy levels $W_{n}$ are therefore a sum of the individual hadron masses and any interaction energy available to impart relative momentum to the final decay products in a corresponding continuum setting. Therefore, the relative momenta $k_{n}$ must be considered continuous variables, just like the $W_{n}$, in the dispersion relation. That is, the $k_{n}$ are not subject to a $2 \pi m / L$ restriction imposed by the lattice. The index $n$ merely numbers the (six) states as they emerge from the simulation. (The point being that even though there is a restriction on allowable total momenta there is no such restriction on masses or mass differences when computed using effective mass functions.)

These momenta are input to Lüscher's formula [49] for the s-wave $(l=0)$ scattering phase shifts $\delta_{0}\left(k_{n}\right)$,

$$
\tan \delta_{0}\left(k_{n}\right)=-\frac{\pi^{3 / 2} q_{n}}{\mathcal{Z}_{00}\left(1 ; q_{n}^{2}\right)} \quad, \quad q_{n}=\frac{k_{n} L_{s}}{2 \pi}
$$

Here $\mathcal{Z}_{00}\left(1 ; q^{2}\right)$ is a generalized $\zeta$-function, see eq. (5.7), and $L_{s}=L a_{s}$ is the physical size of the spatial box using the bare anisotropy, $a_{s}=2 a_{t}$. 
The solution $\delta_{0}$ of eq. (5.17) may be viewed as a function of the continuous variable $q$, i.e. $\delta_{0}=-\arctan \left(\pi^{\frac{3}{2}} q / \mathcal{Z}_{00}\left(1 ; q^{2}\right)\right)$. This function was obtained by interplolating tabular data from [49] in the manner shown in Fig. 12.

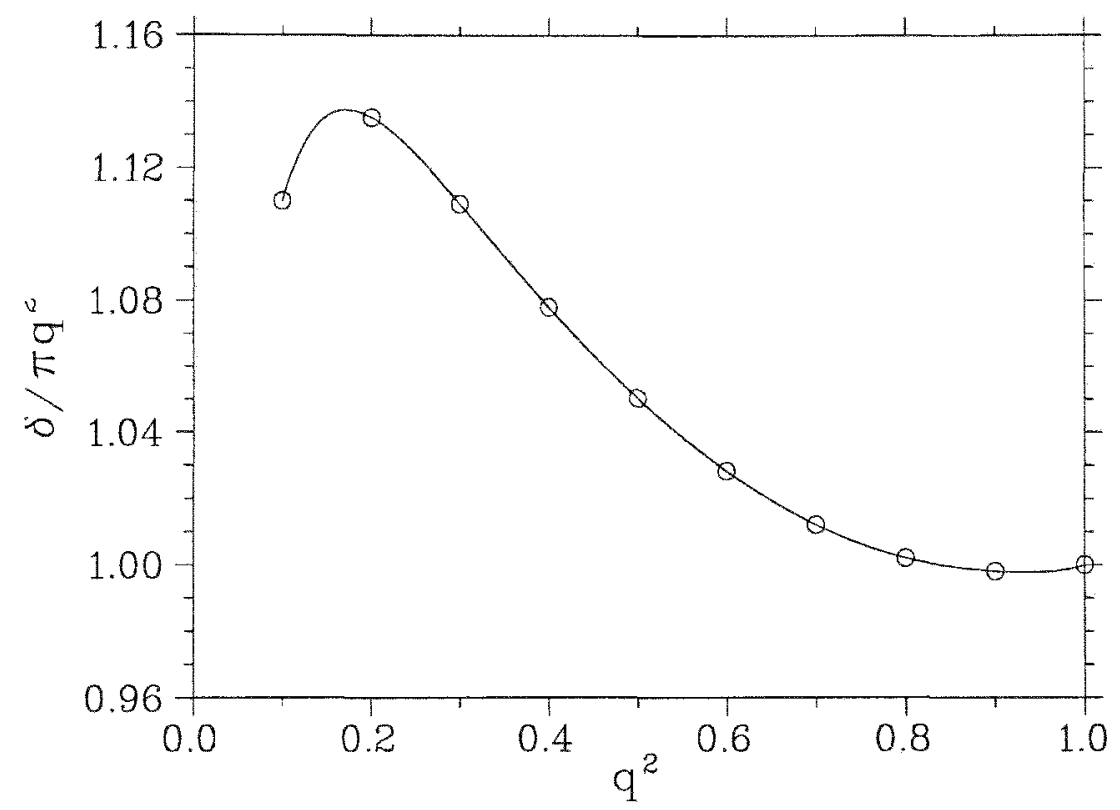

Figure 12: Plot of $\delta_{0} / \pi q^{2}$ versus $q^{2}$ in the range $0.1 \leq q^{2}<1.0$. The line represents a polynomial interpolation of a function table (circles) from [49].

We then attempt fits to all sets of phase shift data with a Breit-Wigner model,

$$
\tan \delta_{0}(k)=\frac{\Gamma / 2}{E_{0}-W(k)}
$$

with

$$
W(k)=\sqrt{m_{\pi}^{2}+k^{2}}+\sqrt{m_{a_{1}}^{2}+k^{2}},
$$

where $E_{0}$ and $\Gamma$ are parameters. When the fit is successful, $\Gamma$ may be interpreted as a decay width and $E_{0}$ as a resonance energy. 


\subsection{The Breit-Wigner function and decay widths.}

As a working definition we adopt the notion that a resonance occurs when then scattering phase angle $\delta_{l}(k)$ passes through $\pi / 2$ as a function of $k$.

The scattering amplitude for the partial wave $l$ is $[80,81]$,

$$
f_{l}=\frac{e^{2 i \delta_{l}}-1}{2 i}=\frac{1}{\cot \left(\delta_{l}\right)-i} \text {. }
$$

One may expand $\cot \left(\delta_{l}\right)$ in a Taylor series,

$$
\cot \left(\delta_{l}(E)\right)=\cot \left(\delta_{l}\left(E_{0}\right)\right)+\left(E-E_{0}\right)\left[\frac{d}{d E} \cot \left(\delta_{l}(E)\right)\right]_{E=E_{0}}+\ldots
$$

about the mass $E_{0}$ of the resonance. Because of $\delta_{l}\left(E_{0}\right)=\frac{\pi}{2}$ we have,

$$
\cot \left(\delta_{l}(E)\right) \approx\left(E_{0}-E\right) \frac{2}{\Gamma}
$$

which is known as the Breit-Wigner form of the equation for the scattering phase shift, and

$$
\Gamma \equiv \frac{2}{\left[\frac{d}{d E}(\cot (\delta(E))]_{E=E_{0}}\right.}
$$

is the width. The full width is a sum of partial widths $\Gamma_{i}$ in, possibly, other open channels $i$,

$$
\Gamma=\sum_{i} \Gamma_{i}
$$




\section{Decay width results}

In order for the decay $h \rightarrow \pi+a_{1}$ to be kinematically allowed we must have

$$
m_{h}=m_{\pi}+m_{a_{1}}+\Delta E
$$

where $\Delta E \geq 0$ is the relative kinetic energy in the decay channel. According to Table $2, a_{t} \Delta E \approx 0.05-0.06$ or $\Delta E \approx 0.15-0.19 \mathrm{GeV}$ using the scale eq. (4.7). The reality of a practical LQCD simulation is, unfortunately, that this region is very hard to reach with feasible lattice parameters because it requires very small quark or pion masses. (In addition the quenched approximation cannot be justified as $m_{\pi} \rightarrow 0$.) In this context we exhibit in Fig. 13 a comparison of the masses $m_{h}$ and $m_{\pi}+m_{a_{1}}$ versus $m_{\pi}^{2}$. An actual level crossing between the $h$ and the non-interacting $\pi+a_{1}$ system occurs only in the extrapolated data at relatively light pion mass, as it would in Nature, giving further credibilty to the simulation. As a side remark, it is interesting to note that the decay $\rho \rightarrow \pi+\pi$ is even more impractical due to the level crossing problem because the decay channel involves two pions in a relative p-wave. The energy of the latter is very large on manageable lattice volumes, practically precluding a level crossing even with extrapolations like Fig. 13. For the $\pi+a_{1}$ system, on the other hand, the decay channel mesons can exist in a relative s-wave. This is the feature that makes the current simulation feasible. 


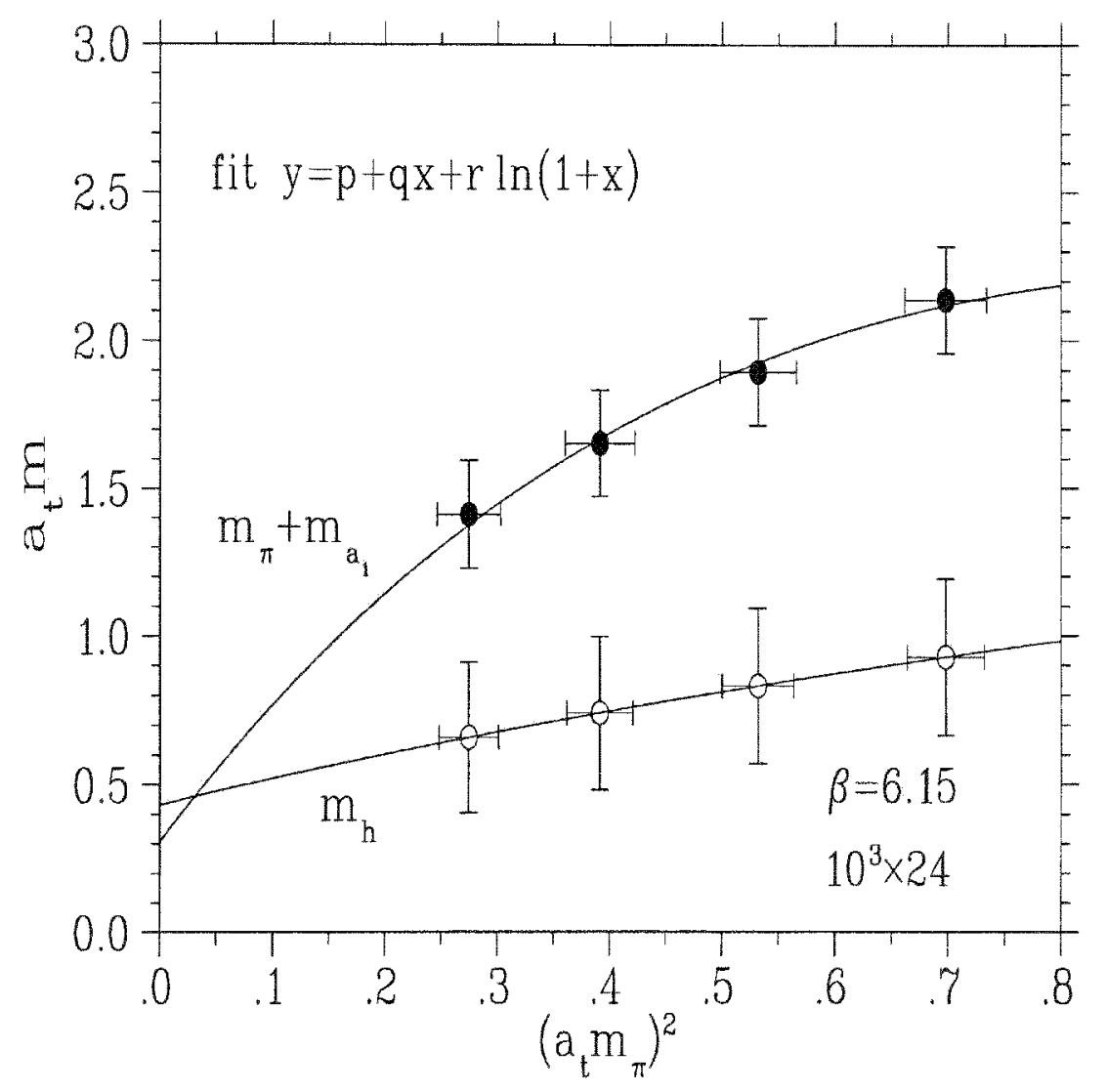

Figure 13: Effective masses for the hybrid meson (unfilled dots) and $a_{1}+\pi$ two meson system (filled dots) on the $10^{3} \times 24$ lattice (2 levels of smearing) This shows an energy level crossing at light pion mass. 


\subsection{Results for scattering phase shifts and decay widths}

In light of the kinematical situation discussed above, two approaches were tried to get the physical decay width. The first approach is to extrapolate the mass spectra to small physical pion mass, use these extrapolated spectra to compute momenta and phase shifts, and then fit a Breit-Wigner function to a plot of phase shifts $\delta_{0}\left(k_{n}\right)$ vs. a discrete set of momenta $k_{n}$. The second approach does not use extrapolated mass spectra. In this approach, the spectra are taken at each of the four pion masses used in the simulation. Momenta and phase shifts are computed for spectra at each pion mass, and then one attempts to fit Breit-Wigner functions for the data at each pion mass. The widths from the Breit-Wigner functions for each pion mass are then plotted against the pion mass, and an extrapolation of the width to small pion mass is attempted.

Phase shift data obtained using the second approach (non-extrapolated spectra) did not match up well to the Breit-Wigner model. The reason being, apparent from Fig. 13, that the pion masses used give rise to spectra quite far from the energy level crossing. Hence, it is unlikely to observe resonance behavior at those pion masses.

The first approach was successful. The extrapolated spectral masses $W_{n}$, relative momenta $k_{n}$, and s-wave scattering phase shifts $\delta_{0}\left(k_{n}\right)$ are listed in Table 4. Missing entries for $k_{n}$ and $\delta_{0}\left(k_{n}\right)$ indicate spectral levels outside the elastic region, see eq. (5.16). Results for two lattice sizes $L^{3} \times 24$ are given. The extrapolations for these lattices are displayed above in Fig. 10. 


\begin{tabular}{|c|c|c|c|c|}
\hline$L$ & $n$ & $a_{t} W_{n}$ & $a_{t} k_{n}$ & $\delta_{0}\left(k_{n}\right)$ \\
\hline 12 & 1 & $.641(156)$ & $.162(14)$ & $74.6(11.6)$ \\
12 & 2 & $.626(245)$ & $.151(18)$ & $65.8(14.1)$ \\
12 & 3 & $.187(45)$ & - & - \\
12 & 4 & $.468(112)$ & $.027(11)$ & $2.1(1.8)$ \\
12 & 5 & $.309(105)$ & - & - \\
12 & 6 & $.255(45)$ & - & - \\
\hline 10 & 1 & $.663(214)$ & $.198(15)$ & $77.1(10.5)$ \\
10 & 2 & $.614(27)$ & $.156(10)$ & $50.0(6.1)$ \\
10 & 3 & $.489(43)$ & $.055(14)$ & $6.0(3.1)$ \\
10 & 4 & $.440(103)$ & $.010(10)$ & $0.2(0.5)$ \\
10 & 5 & $.364(98)$ & - & - \\
10 & 6 & $.333(43)$ & - & - \\
\hline
\end{tabular}

Table 4: Extrapolated energy spectra $W_{n}, n=1 \ldots 6$ with resulting momenta and phase shifts of the $\pi a_{1}$ system for two lattice dimensions $L$. The entries for $a_{t} k_{n}$ and $\delta_{0}\left(k_{n}\right)$ that are blank correspond with energy spectra outside of the elastic region.

Fitting of Breit-Wigner functions in principle can be done in a scale independent way using the model

$$
\delta_{0}(k)=\arctan \left(\frac{\left(a_{t} \Gamma\right) / 2}{a_{t} E_{0}-\sqrt{\left(a_{t} m_{\pi}\right)^{2}+\left(a_{t} k\right)^{2}}-\sqrt{\left(a_{t} m_{a_{1}}\right)^{2}+\left(a_{t} k\right)^{2}}}\right)
$$

at fixed $a_{t} m_{\pi}=0$ where $a_{t} \Gamma$ and $a_{t} E_{0}$ are the fit parameters, and $a_{t} m_{a_{1}}$ is taken from Table 2. Fits were attempted employing a standard Levenberg-Marquardt algorithm [82]. However, the phase shift data is very sparse. While the fits returned stable results for the resonance energy parameter $a_{t} E_{0}$, the width parameter $a_{t} \Gamma$, being an indicator for a derivative, is not well determined by the algorithm. 
An alternative analysis procedure is based on the observation that the phase shift data are clustered around two values of $a_{t} k$, namely $\approx 0.17$ and $\approx 0.04$, averaged over the $L=12$ and $L=10$ lattices, see Table 4 . This suggests that no more than two distinct physical states are uncovered by the simulation. From Table 4, denoting the weighted $\left(\chi^{2}=\min \right)$ averages of the clustered momenta by $\vec{k}_{1,2}$, the corresponding energies by $\omega_{1,2}=a_{t} W\left(\bar{k}_{1,2}\right)$, and $\tau_{1,2}=\tan \delta_{0}\left(\bar{k}_{1,2}\right)$ via eq. (5.17), we obtain a set of two equations from eq. (5.18)

$$
\tau_{i}=\frac{a_{t} \Gamma / 2}{a_{t} E_{0}-\omega_{i}}
$$

for $i=1,2$. These are solved by

$$
a_{t} \Gamma=2\left(\omega_{1}-\omega_{2}\right) \frac{\tau_{1} \tau_{2}}{\tau_{1}+\tau_{2}}
$$

and

$$
a_{t} E_{0}=\frac{\omega_{2} \tau_{2}-\omega_{1} \tau_{1}}{\tau_{2}-\tau_{1}}
$$

The results are listed in Table 5, and using these paramters in eq. (6.2), lead to the Breit-Wigner phase shift curves shown in Fig. 14 for the two lattice sizes. 
The uncertainties for $a_{t} \Gamma$ and $a_{t} E_{0}$ are based on the statistical jackknife [73] errors for $a_{t} k$ as listed in Table 4. Those give rise to errors $\Delta \bar{k}_{1,2}$ for the weighted momentum averages $\bar{k}_{1,2}$. The analysis procedure described above was repeated many times with momenta $k_{1,2}=\bar{k}_{1,2}+x \cdot \Delta \bar{k}_{1,2}$ where $x_{1,2}$ are normal distributed random numbers with variance one. Also the $a_{1}$ meson mass, which enters the fit model via eq. (6.2), was subjected to the same randomization. The errors given in Table 5 are the standard deviations resulting from the randomization. Table 5 also contains the physical values for the decay width and the resonance energy using the $\rho$ meson to set the scale, cf. eq. (4.7). Setting the scale with the $a_{1}$ meson, cf. eq. (4.8), results in widths of $39(29) \mathrm{MeV}$ and $108(48) \mathrm{MeV}$ for the $12^{3} \times 24$ and $10^{3} \times 24$ lattices repectively.

\begin{tabular}{|c|c|c|c|c|}
\hline$L$ & $a_{t} \Gamma$ & $a_{t} E_{0}$ & $\Gamma[\mathrm{MeV}]$ & $E_{0}[\mathrm{GeV}]$ \\
\hline 12 & $0.0116(87)$ & $0.625(28)$ & $35(26)$ & $1.88(8)$ \\
10 & $0.0324(143)$ & $0.618(30)$ & $97(43)$ & $1.84(9)$ \\
\hline
\end{tabular}

Table 5: Results for decay widths $\Gamma$ and resonant energies $E_{0}$ for two lattice sizes $L$ without a scale and with a scale set by the $\rho$ mass.

The dashed lines in the two panels of Fig. 14 were obtained using only one of the two data points, at large momenta, in each case. Those result in error bounds for $\Gamma$ of 33-36 MeV and 87-119 MeV for the $12^{3} \times 24$ and $10^{3} \times 24$ lattices repectively. 
Finally, Fig. 15 shows the combined phase shift data from the two lattices. This is permissible because the $\rho$ mass based scales are very close $\left(a_{t}=0.332\right.$ and $\left.0.335 \mathrm{GeV}^{-1}\right)$. The Breit-Wigner fit is based on averaging over two and four data points (in each cluster), resulting in $\Gamma=58(24) \mathrm{MeV}$ and $E_{0}=1.87(6) \mathrm{GeV}$. 

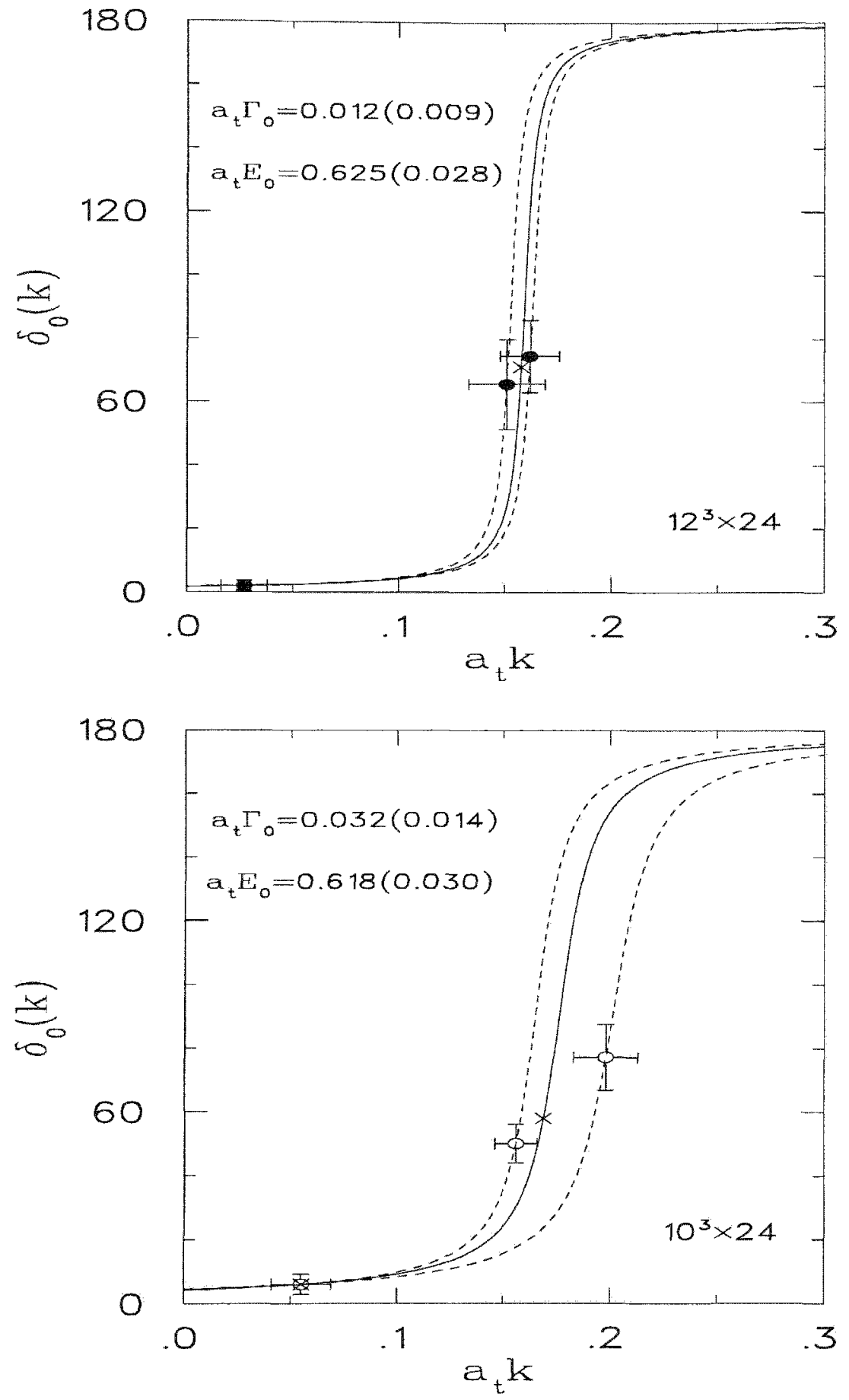

Figure 14: Resonant scattering phase shifts $\delta_{0}\left(k_{n}\right)$ from the $1^{-+}$hybrid exotic meson and $\pi+a_{1}$ meson-meson operators using only extrapolated spectra from the $12^{3} \times 24$ (filled dots, upper panel) and $10^{3} \times 24$ (unfilled dots, lower panel) lattices. The solid curves are Breit-Wigner model interpolations according to eq's (6.4) and (6.5). The crosses indicate the $\chi^{2}$ weighted averages over data points. 


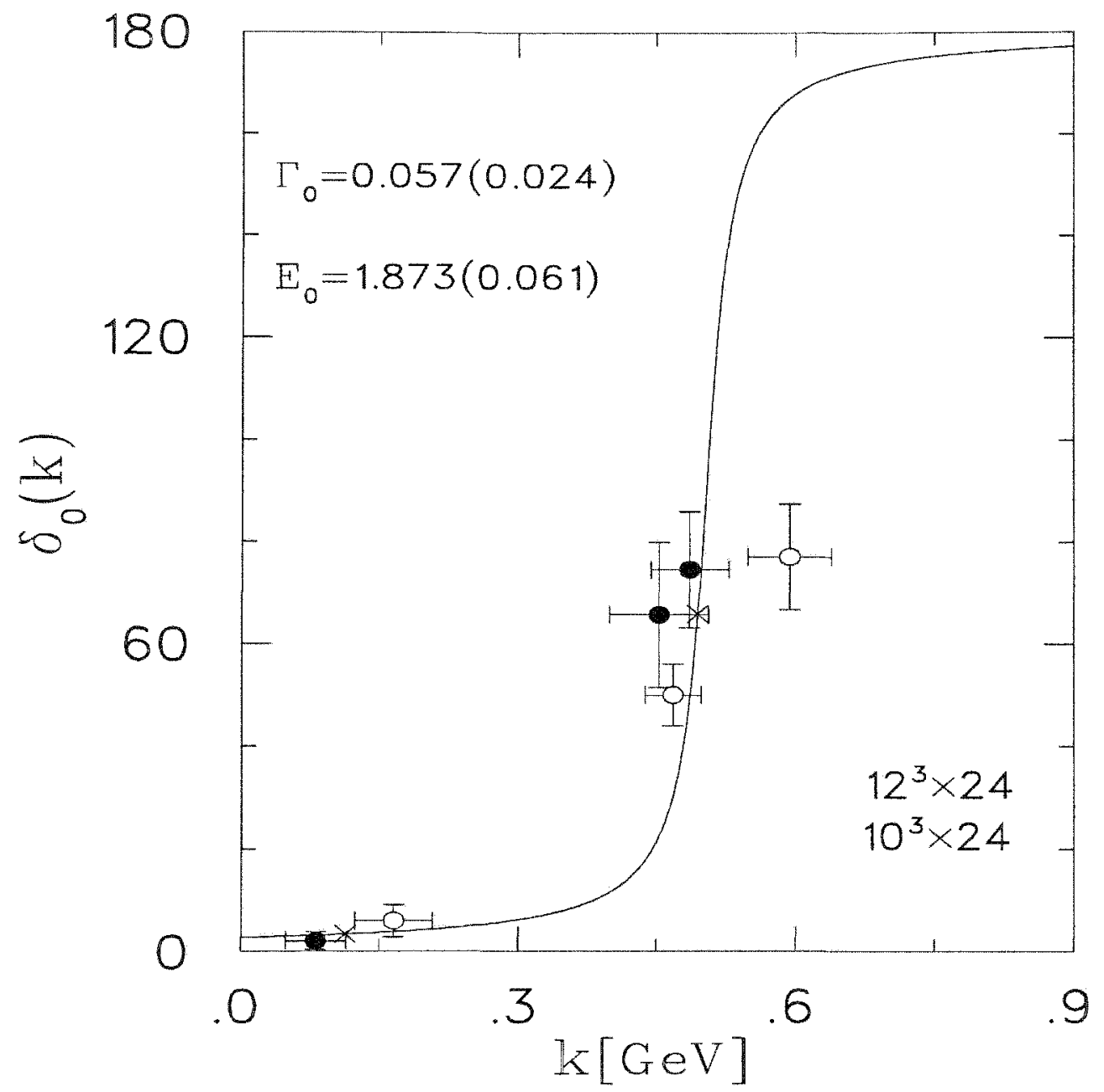

Figure 15: Resonant scattering phase shifts $\delta_{0}\left(k_{n}\right)$ from the $1^{-+}$hybrid exotic meson and $\pi+a_{1}$ meson-meson operators using only extrapolated spectra from the $12^{3} \times$ 24 (filled dots) and $10^{3} \times 24$ (unfilled dots) lattices combined. The solid curve is a Breit-Wigner model interpolation according to eq's (6.4) and (6.5). The crosses indicate the $\chi^{2}$ weighted averages over data points. 


\subsection{Systematic errors}

The effects of systematic errors on the results, $\Gamma$ and $E_{0}$, of this simulation are difficult to assess. In principle this can only be done by repeating it with various different choices of parameters. Probably the largest source of systematic error, stems from curve fitting and extrapolation techniques. All extrapolations were done with either a logarithmic term, cf. eq. (4.6), or an $x^{3 / 2}$ term in the fit, and these terms produce similar results. When these terms are removed, the resulting linearly extrapolated mass spectra from the $6 \times 6$ correlation matrix will shift upwards, but the extrapolated masses from single meson correlators will not change as much. The end result is a slight increase in momenta, a slighly larger phase shift, and a decay width increasing on the order of $10 \mathrm{MeV}$.

Another source of systematic error is fitting of the Breit-Wigner functions. Given the sparsity of data points it is not at all clear that the physical phase shifts will indeed follow a Breit- Wigner form. In order to resolve this problem the simulation would have to be repeated at several values of the gauge coupling $\beta$, thus mapping out some sort of continuous curve $\delta_{0}(k)$ vs. $k$, and then attempting Breit-Wigner fits. The results of this work do rely on the a priori assumption that the simulation data follow a Breit-Wigner model. On the other hand, adopting the less strigent criteria that a resonance is present if the phase shift data passes through $90 \mathrm{deg}$, the simulation results clearly indicate the presence of such. This, in itself, is a remarkable outcome of this project. Although this does not help putting bounds on the systemmatic error of $\Gamma$, it does appear that results for the resonance energy $E_{0}$ are somewhat more reliable. 
Systematic errors are also caused by finite size effects. At first sight, judging by the small difference of the $\rho$ meson masses on the $L=12$ and $L=10$ lattices (see Table 2) those appear to be small. Finite size effects should be expected to be much larger for larger-sized hadrons like the $a_{1}$ for example. This is particularly true for two-hadron systems studied in this work. For example, the spectra displayed in Fig. 10 are significantly different, particularly for excited states on the $L=12$ and $L=10$ lattices, their size though being quite simular. Again these effects can only be studied by repeating this simulation with several lattices of different sizes.

Obtaining a single scattering phase data point requires evaluating up to three effective mass functions - one for the correlator matrix eigenvalue, one for the $a_{1}$ meson, and one for the $\pi$ meson. The variability in choosing which time slices of the correlaton functions to use in fitting effective masses produces a variability in the decay width. Here, one usually wants to maximize the plateau width of the effective mass functions to optimize the statistical error. Reducing the plateau width to estimate a systematic error is of limited value. An alternative analysis technique is Bayesian inference, e.g. the maximum entropy method, which yields mass spectral functions. Examples are shown in Appendix G. 


\section{$7 \quad$ Summary and Conclusion}

Decay properties of a hybrid exotic meson $h$ with $J^{P C}=1^{-+}$were computed using quenched LQCD and Lüscher's method to extract contiuum scattering phase shifts from quantum fields in a finite box. Spectral data were extrapolated to the physical pion mass region, $m_{\pi} \rightarrow 0$. Assuming a Breit-Wigner model, decay widths average about $60 \mathrm{MeV}$ with statistical errors of about $20 \mathrm{MeV}$ using combined extrapolated energy spectra from both the $12^{3} \times 24$ and $10^{3} \times 24$ lattices. For those lattices, taken one at a time, the decay widths range from $35-100 \mathrm{MeV}$. These widths are obtained using the $\rho$ meson to set the scale. If the $a_{1}$ meson sets the scale, then the widths for these two lattices are in the range $40-110 \mathrm{MeV}$ with statistical errors of about $30-50 \mathrm{MeV}$.

The number of data points available to fit Breit-Wigner functions is very sparse, the reason being that many energy levels fell outside of the elastic region where phase shifts using Lüscher's formulae cannot be computed. To overcome this limitation, a larger correlation matrix will have to be used. Expanding this matrix can be done by adding more smearing levels, and spatially extended operators, in the individual correlators or possibly by using a coupled channel type analysis in which more than one decay channel is represented in the matrix. More importantly, several values of the coupling parameter $\beta$ need to be employed in order to have more phase shift data available. This should be the next step improving on the results of this work. 
Using the $\rho$ meson to set the scale, the resonance mass of the hybrid meson in this simulation is $1.9 \pm 0.1 \mathrm{GeV}$. Using the $a_{1}$ meson to set the scale, the hybrid mass is $2.1 \pm 0.1 \mathrm{GeV}$. In contrast to the decay width, the resonance mass turned out to be well determined by this LQCD simulation.

Historically, hadron mass calculations within LQCD were typically based on using single-hadron operators, ignoring the fact that most hadrons are (unstable) resonances and thus decay [47]. We have, in this project, taken seriously this aspect of hadron physics. While the numerical values for the decay width, in particular, should be taken as a guide, the approach of studying hadrons as resonances using Lüscher's method should be given serious consideration for future studies with LQCD. 


\section{References}

[1] M. J. Herrero. The standard model, http://arxiv.org/abs/hep-ph/9812242.

[2] Rajan Gupta. Introduction to Lattice QCD. Los Alamos National Laboratory, Los Alamos, NM, 2003.

[3] S. Eidelman et al. Review of particle physics. Phys. Lett, B592, 2004.

[4] F.E. Close. An Introduction to Quarks and Partons. Academic Press, New York, NY, 1979.

[5] Nathan Isgur and Gabriel Karl. Positive parity excited baryons in a quark model with hyperfine interactions. Phys. Rev., D19:2653, 1979. Erratumibid.D23:817,1981.

[6] Nathan Isgur and Gabriel Karl. P wave baryons in the quark model. Phys. Rev., D18:4187, 1978.

[7] Nathan Isgur and Jack Paton. A flux tube model for hadrons in qcd. Phys. Rev., D31:2910, 1985.

[8] Nathan Isgur and Jack Paton. A flux tube model for hadrons. Phys. Lett, B124:247, 1983.

[9] Nathan Isgur, Richard Kokoski, and Jack Paton. Gluonic excitations of mesons: Why they are missing and where to find them. Phys. Rev. Lett., 54:869, 1985.

[10] Nathan Isgur. Flux tube zero-point motion, hadronic charge radii, and hybrid meson production cross sections. Phys. Rev., D60:114016, 1999.

[11] Ted Barnes. Photoproduction of hybrid mesons. 1998.

[12] Frank E. Close and Philip R. Page. The production and decay of hybrid mesons by flux tube breaking. Nucl. Phys., B443:233-254, 1995.

[13] Ted Barnes. Hybrid baryons: A brief review. 2000. nucl-th/0009011.

[14] http://dustbunny.physics.indiana.edu/HallD/Welcome.html.

[15] http://wwwcompass.cern.ch/.

[16] http://www.Ins.cornell.edu/public/CLEO/spoke/ CLEOc/jima.html.

[17] D.R. Thompson et al. Evidence for exotic meson production in the reaction $\pi^{-} p \rightarrow \eta \pi^{-} p$ at $18 \mathrm{gev} / \mathrm{c}$. Phys. Rev. Lett., 79(9):1630, 1997. 
[18] S. U. Chung et al. Evidence for exotic $\mathrm{j}(\mathrm{pc})=1-+$ meson production in the reaction pi- $\mathrm{p}$ to eta pi- $\mathrm{p}$ at 18-gev/c. Phys. Rev., D60:092001, 1999.

[19] A. Abele et al. Exotic eta pi state in anti-p d annihilation at rest into pi- pi0 eta p(spectator). Phys. Lett., B423:175-184, 1998.

[20] G. T. Condo et al. Photoproduction of an isovector rho pi state at $1775-\mathrm{mev}$. Phys. Rev., D43:2787-2791, 1991.

[21] G. S. Adams et al. Observation of a new $\mathrm{j}(\mathrm{pc})=1-+$ exotic state in the reaction pi p to pi+ pi- pi-p at 18-gev/c. Phys. Rev. Lett., 81:5760-5763, 1998.

[22] E. I. Ivanov et al. Observation of exotic meson production in the reaction pip to eta' pi- p at 18-gev/c. Phys. Rev. Lett., 86:3977-3980, 2001.

[23] D. J. Gross and F. Wilczek. Asymptotically free gauge theories. Phys. Rev., D8:3633, 1973.

[24] H. D. Politzer. Reliable perturbative results for strong interactions. Phys. Rev. Let., 30:1346, 1973.

[25] K. G. Wilson. Confinement of quarks. Phys. Rev., D10:2445-2459, 1974.

[26] M. Creutz. Monte carlo study of quantized su(2) gauge theory. Phys. Rev, D21:2308, 1980.

[27] H. Hamber and G. Parisi. Phys. Rev. Lett., 47:1792, 1981.

[28] D. Weingarten. Phys, Lett., B109:57, 1981.

[29] K. J. Juge, J. Kuti, and C. J. Morningstar. Ab initio study of hybrid anti-b g b mesons. Phys. Rev. Lett., 82:4400-4403, 1999.

[30] K. J. Juge, J. Kuti, and C. J. Morningstar. Gluon excitations of the static quark potential and the hybrid quarkonium spectrum. Nucl. Phys. Proc. Suppl., 63:326-331, 1998.

[31] S. Perantonis and C. Michael. Static potentials and hybrid mesons from pure su(3) lattice gauge theory. Nucl. Phys., B347:854-868, 1990.

[32] Tommy Burch, Kostas Orginos, and Doug Toussaint. Measurement of hybrid content of heavy quarkonia using lattice nrqcd. Phys. Rev., D64:074505, 2001.

[33] I. T. Drummond, N. A. Goodman, R. R. Horgan, H. P. Shanahan, and L. C. Storoni. Spin effects in heavy hybrid mesons on an anisotropic lattice. Phys. Lett., B478:151-160, 2000. 
[34] T. Manke, I. T. Drummond, R. R. Horgan, and H. P. Shanahan. Hybrid quarkonia with high statistics from nrqcd. Nucl. Phys. Proc. Suppl., 63:332334, 1998.

[35] C. McNeile et al. Exotic meson spectroscopy from the clover action at beta $=$ 5.85 and 6.15. Nucl. Phys. Proc. Suppl., 73:264-266, 1999.

[36] Claude W. Bernard et al. Exotic mesons in quenched lattice qcd. Phys. Rev., D56:7039-7051, 1997.

[37] Claude W. Bernard et al. Exotic hybrid mesons with light quarks. Nucl. Phys. Proc. Suppl., 53:228-231, 1997.

[38] P. Lacock and K. Schilling. Hybrid and orbitally excited mesons in full qcd. Nucl. Phys. Proc. Suppl., 73:261-263, 1999.

[39] P. Lacock, C. Michael, P. Boyle, and P. Rowland. Hybrid and orbitally excited mesons in quenched qcd. Nucl. Phys. Proc. Suppl., 63:203-205, 1998.

[40] P. Lacock, C. Michael, P. Boyle, and P. Rowland. Hybrid mesons from quenched qcd. Phys. Lett., B401:308-312, 1997.

[41] P. Lacock, C. Michael, P. Boyle, and P. Rowland. Orbitally excited and hybrid mesons from the lattice. Phys. Rev., D54:6997-7009, 1996.

[42] C. Bernard et al. Exotic hybrid mesons from improved kogut-susskind fermions. Nucl. Phys. B (Proc. Suppl.), 119:260, 2003.

[43] J. N. Hedditch et al. Light-quark flic fermion simulations of the 1-+ exotic meson. PoS, LAT2005:040, 2005.

[44] C. McNeile, C. Michael, and P. Pennanen. Hybrid meson decay from the lattice. Phys. Rev., D65:094505, 2002.

[45] L. Maiani and M. Testa. Final state interactions from euclidean correlation functions. Phys. Lett., B245:585-590, 1990.

[46] C. Michael. Particle decay in lattice gauge theory. Nucl. Phys., B327:515, 1989.

[47] C. Michael. Hadronic decays. In Proceedings of The 23rd International Symposium on Lattice Field Theory, 25-30 July 2005, Trinity College, Dublin, Ireland, PoS(LAT2005)008, 2005.

[48] Thomas A. DeGrand. Resonance masses from monte carlo simulations. Phys. Rev., D43:2296-2300, 1991. 
[49] M. Lüscher. Signatures of unstable particles in finite volume. Nucl. Phys., B364:237-254, 1991.

[50] L. Lellouch and M. Lüscher. Weak transition matrix elements from finitevolume correlation functions. Commun. Math. Phys., 219:31-44, 2001.

[51] M. Lüscher. Two particle states on a torus and their relation to the scattering matrix. Nucl. Phys., B354:531-578, 1991.

[52] M. Lüscher and U. Wolff. How to calculate the elastic scattering matrix in two-dimensional quantum field theories by numerical simulation. Nucl. Phys., B339:222-252, 1990.

[53] M. Göckeler, H. A. Kastrup, J. Westphalen, and F. Zimmermann. Scattering phases on finite lattices in the broken phase of the four-dimensional o(4) $\phi^{4}$ theory. Nucl. Phys., B425:413-448, 1994.

[54] F. Zimmermann, J. Westphalen, M. Göckeler, and H. A. Kastrup. Unstable particles in finite volume: The broken phase of the 4-d o(4) nonlinear sigma model. Nucl. Phys. Proc. Suppl., 30:879-882, 1993.

[55] H. R. Fiebig, A. Dominguez, and R. M. Woloshyn. Meson meson scattering phase shifts in (2+1)-dimensional lattice qed. Nucl. Phys., B418:649-685, 1994.

[56] C.R. Gattringer and C.B. Lang, Nucl. Phys. B 391 (1993) 463.

[57] C.R. Gattringer, I. Hip and C.B. Lang, Nucl.Phys. B (Proc. Suppl.) 30 (1993) 875.

[58] M. Guagnelli, E. Marinari, and G. Parisi. Scattering lengths from fluctuations. Phys. Lett., B240:188, 1990.

[59] R. Gupta, A. Patel, and S. R. Sharpe. I = 2 pion scattering amplitude with wilson fermions. Phys. Rev., D48:388-396, 1993.

[60] Chuan Liu, Jun-hua Zhang, Ying Chen, and J. P. Ma. Calculating the $\mathrm{i}=2$ pion scattering length using tadpole improved clover wilson action on coarse anisotropic lattices. Nucl. Phys., B624:360-376, 2002.

[61] M. Lüscher. Volume dependence of the energy spectrum in massive quantum field theories. 2. scattering states. Commun. Math. Phys., 105:153-188, 1986.

[62] H. W. Hamber, E. Marinari, G. Parisi, and C. Rebbi. Considerations on numerical analysis of qcd. Nucl. Phys., B225:475, 1983. 
[63] M. Peskin and D. Schroeder. An Introduction to Quantum Field Theory. Westview Press, Boulder,CO, 1995.

[64] I. Montvay and G. Münster. Quantum Fields on the Lattice. Cambridge University Press, Cambridge, UK, 1994.

[65] J. B. Kogut and L. Susskind. Hamiltonian formulation of wilson's lattice gauge theories. Phys. Rev., D11:395, 1975.

[66] K. G. Wilson. In A. Zichichi, editor, New Phenomena in Subnuclear Physics, page 69. Plenum Press, New York, 1975.

[67] C. Bernard et al. Exotic mesons in quenched lattice qcd. Phys. Rev., D56:7040$7041,1997$.

[68] C. Alexandrou, S. Güsken, F. Jegerlehner, K. Schilling, and R. Sommer. The static approximation of heavy - light quark systems: A systematic lattice study. Nucl. Phys., B414:815-855, 1994.

[69] C. Albanese and et al. Phys. Lett., B192:163, 1987.

[70] M. Creutz, Quarks, Gluons and Lattices, Cambridge University Press (Cambridge 1983).

[71] N. Cabibbo and E. Marinari. A new method for updating su(n) matrices in computer simulations of gauge theories. Phys. Lett., 119B:387, 1982.

[72] Andreas Frommer, Bertold Nöckel, Stephan Güsken, Thomas Lippert, and Klaus Schilling. Many masses on one stroke: Economic computation of quark propagators. Int. J. Mod. Phys., C6:627-638, 1995.

[73] B. Efron, SIAM Review 21 (1979) 460.

[74] In Aron M. Bernstein and Barry R. Holstein, editors, Chiral Dynamics: Theory and Experiment, volume 452 of Lecture Notes in Physics. Springer-Verlag, Berlin, Heidelberg, New York, 1995.

[75] Jan Smit. Introduction to Quantum Fields on a Lattice. Cambridge University Press, Cambridge,UK, 2002.

[76] S. V. Wright et al. Hadron mass extraction from lattice qcd. Nucl. Phys. Proc. Suppl, 109A:50-54, 2002.

[77] D. Alde et al. Evidence for a $1^{-+}$exotic meson. Phys. Lett. B, 205(2,3):397, 1988. 
[78] A. Abele et al. Exotic $\eta \pi$ state in antiproton-deuterium annihilation at rest into $\pi^{-} \pi^{0} \eta p$. Phys. Lett., B423:175, 1998.

[79] H. R. Fiebig and H. Markum. Interactions between lattice hadrons. In A. M. Green, editor, Hadronic Physics from Lattice QCD, volume 9 of International Review of Nuclear Physics, chapter 5. World Scientific, Singapore, 2002. submitted to the editor.

[80] J. R. Taylor. Scattering Theory. Wiley, New York, 1972.

[81] A. Messiah. Quantum Mechanics - Vol. II. North-Holland, Amsterdam, 1966.

[82] Numerical Recipes in Fortran 77. Cambridge University Press, Cambridge,UK, 1995.

[83] The Royal Swedish Academy of Sciences. Asymptotic freedom and quantum chromodynamics. AAPPS Bulletin, 14(6):7-8, 2004.

[84] H. R. Fiebig. Spectral density analysis of time correlation functions in lattice qcd using the maximum entropy method. Phys. Rev., D65:094512, 2002. 


\section{Appendices}

\section{A. Rational for diagonalization of correlator matrix}

Getting an energy spectrum requires finding the eigenvalues of a correlation matrix. To find these eigenvalues, we convert from a matrix of complex numbers to one of real numbers.

We can represent the hermitian correlation matrix as a sum of two matices.

$$
C=A+i B=C^{\dagger}=A^{\dagger}-i B^{\dagger}
$$

Therefore,

$$
A^{T}=A \quad \text { and } \quad B^{T}=-B .
$$

The eigenvalue equation is,

$$
C x=\lambda x \quad \text { where } \quad x=u+i v
$$

Substituting for $\mathrm{C}$ and $\mathrm{x}$,

$$
\begin{gathered}
(A+i B)(u+i v)=\lambda(u+i v) \\
A u-B v+i(B u+A v)=\lambda u+i \lambda v
\end{gathered}
$$

This means,

$$
A u-B v=\lambda u \quad \text { and } \quad B u+A v=\lambda v
$$

From the $6 \times 6$ original complex matrix a $12 \times 12$ real antisymmetric matrix is formed,

$$
\left(\begin{array}{cc}
A & -B \\
B & A
\end{array}\right)\left(\begin{array}{l}
u \\
v
\end{array}\right)=\lambda\left(\begin{array}{l}
u \\
v
\end{array}\right) .
$$

Because we now have a real matrix, a standard Jacobi Rotation program can solve for the twelve eigenvalues. 
These twelve eigenvalues are two-fold degenerate.

$$
\begin{array}{r}
(A+i B)(u+i v)=\lambda(u+i v) \rightarrow(A+i B)(-v+i u)=\lambda(-v+i u) . \\
\left(\begin{array}{cc}
A & -B \\
B & A
\end{array}\right)\left(\begin{array}{c}
-v \\
u
\end{array}\right)=\lambda\left(\begin{array}{l}
u \\
v
\end{array}\right) .
\end{array}
$$

Giving a two-fold degenerate pair of orthogonal eigenvectors,

$$
\left(\begin{array}{l}
u \\
v
\end{array}\right),\left(\begin{array}{c}
-v \\
u
\end{array}\right)
$$

where,

$$
\begin{aligned}
& \left(\begin{array}{l}
u \\
v
\end{array}\right)^{T^{T}}\left(\begin{array}{c}
-v \\
u
\end{array}\right)=-u^{T} v+v^{T} u \\
& =-\sum_{i} u_{i} v_{i}+\sum_{i} v_{i} u_{i}=0 .
\end{aligned}
$$


B. Gell-Mann Matrices from [64]

$$
\begin{array}{ll}
\lambda_{1}=\left(\begin{array}{lll}
0 & 1 & 0 \\
1 & 0 & 0 \\
0 & 0 & 0
\end{array}\right), & \lambda_{2}=\left(\begin{array}{ccc}
0 & -i & 0 \\
i & 0 & 0 \\
0 & 0 & 0
\end{array}\right), \\
\lambda_{3}=\left(\begin{array}{ccc}
1 & 0 & 0 \\
0 & -1 & 0 \\
0 & 0 & 0
\end{array}\right), \quad \lambda_{4}=\left(\begin{array}{lll}
0 & 0 & 1 \\
0 & 0 & 0 \\
1 & 0 & 0
\end{array}\right), \\
\lambda_{5}=\left(\begin{array}{ccc}
0 & 0 & -i \\
0 & 0 & 0 \\
i & 0 & 0
\end{array}\right), \quad \lambda_{6}=\left(\begin{array}{lll}
0 & 0 & 0 \\
0 & 0 & 1 \\
0 & 1 & 0
\end{array}\right), \\
\lambda_{7}=\left(\begin{array}{ccc}
0 & 0 \\
0 & 0 & -i \\
0 & i & 0
\end{array}\right), \\
\lambda_{8}=\left(\begin{array}{lll}
\frac{1}{\sqrt{3}} & 0 & 0 \\
0 & \frac{1}{\sqrt{3}} & 0 \\
0 & 0 & \frac{-2}{\sqrt{3}}
\end{array}\right) .
\end{array}
$$


C. Euclidean Dirac Matrices (UKQCD convention)

$$
\gamma_{1}=\left(\begin{array}{cccc}
0 & 0 & 0 & i \\
0 & 0 & i & 0 \\
0 & -i & 0 & 0 \\
-i & 0 & 0 & 0
\end{array}\right), \quad \gamma_{2}=\left(\begin{array}{cccc}
0 & 0 & 0 & 1 \\
0 & 0 & -1 & 0 \\
0 & -1 & 0 & 0 \\
1 & 0 & 0 & 0
\end{array}\right),
$$$$
\gamma_{3}=\left(\begin{array}{cccc}
0 & 0 & i & 0 \\
0 & 0 & 0 & -i \\
-i & 0 & 0 & 0 \\
0 & i & 0 & 0
\end{array}\right), \quad \gamma_{4}=\left(\begin{array}{cccc}
1 & 0 & 0 & 0 \\
0 & 1 & 0 & 0 \\
0 & 0 & -1 & 0 \\
0 & 0 & 0 & -1
\end{array}\right),
$$

$$
\gamma_{5}=\gamma_{1} \gamma_{2} \gamma_{3} \gamma_{4}=\left(\begin{array}{cccc}
0 & 0 & 1 & 0 \\
0 & 0 & 0 & 1 \\
1 & 0 & 0 & 0 \\
0 & 1 & 0 & 0
\end{array}\right)=\gamma_{5}^{\dagger}
$$

Charge conjugation matrix, $\mathcal{C}=\gamma_{2} \gamma_{4}$ 


\section{Quark field color transformations}

Quarks exist as any of one six flavors (up, down, strange, charmed, bottom, and top) and they are assigned a baryon number that is conserved ( $\frac{1}{3}$ for quarks (q), $-\frac{1}{3}$ for anti-quarks $(\bar{q})$ ). Quarks also exist as any one of three primary colors (red (r), green (g), or blue (b)), and anti-quarks exist as any one of three anti-primary colors (anti-red $(\bar{r})$, anti-green $(\bar{g})$, or anti-blue $(\bar{b})$ )

6 flavors $\times 3$ colors $=18$ distinct quarks or anti-quarks

Quarks form bound states that are color neutral or white. These white states are created by mixing all three primary colors or by mixing one primary color with it's anti-primary color. Three quarks (qqq), therefore, can form into a particles called baryons and two quarks $(q \bar{q})$ can form into particles called mesons. Four and five quark states are also allowed.

Baryons and mesons comprise a group of particles called hadrons, the group upon which the nuclear strong force acts. Quarks interact by exchanging gluons. Gluons are electrically neutral but not color neutral. They carry one primary color and one anti-primary color $(r \bar{b}, r \bar{g}, g \bar{r}$, etc.). There are nine possible combinations, but one of these is equated to white and is excluded, leaving eight possible combinations, or eight gluons. The three types of elementary interaction vertices for quarks and gluons are displayed in Fig. 16.

Quarks change color during the exchange of gluons. Inside a proton, for example, a green quark changes it's color to red by emitting a gluon having green and anti-red colors (see Fig.17). This gluon is then absorbed by a red quark, whereby the red on the quark is annihilated by the anti-red in the gluon leaving the second quark green. The final color state of the proton, however, remains neutral.

These quark color transformations can be represented by linear transformations. If a three component vector represents the quark color state $(\vec{c})$, 


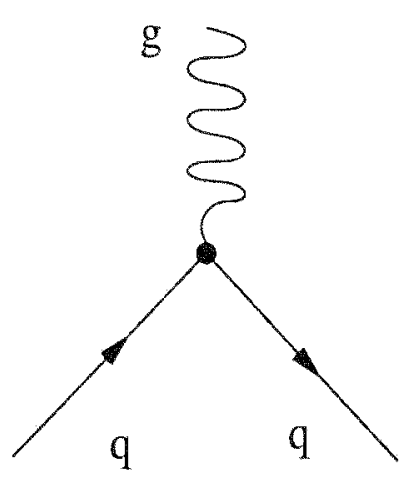

(a)

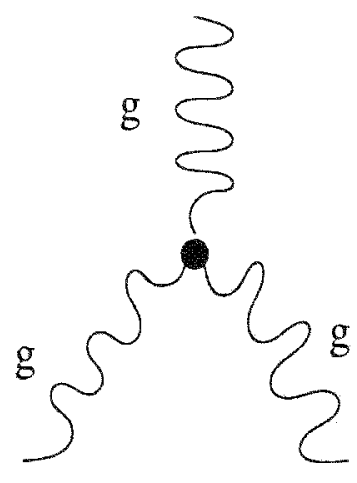

(b)

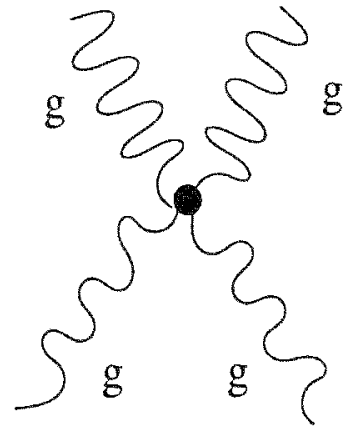

(c)

Figure 16: Three elementary vertices of QCD. (a) quark-gluon, (b) 3-gluon, (c) 4-gluon.

$$
\vec{c}=\left(\begin{array}{l}
r \\
b \\
g
\end{array}\right)
$$

then the change from red $(\vec{r})$ to green $(\vec{g})$ is described by,

$$
\begin{gathered}
\frac{1}{2}\left(\lambda_{4}-i \lambda_{5}\right) \vec{r}=\vec{g} \\
=\frac{1}{2}\left(\left(\begin{array}{lll}
0 & 0 & 1 \\
0 & 0 & 0 \\
1 & 0 & 0
\end{array}\right)-i\left(\begin{array}{ccc}
0 & 0 & -i \\
0 & 0 & 0 \\
i & 0 & 0
\end{array}\right)\right)\left(\begin{array}{l}
1 \\
0 \\
0
\end{array}\right)=\left(\begin{array}{l}
0 \\
0 \\
1
\end{array}\right)
\end{gathered}
$$




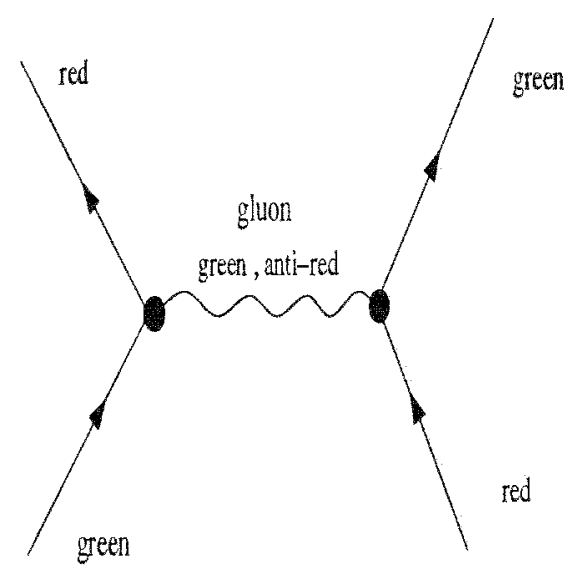

Figure 17: An example of a quark color change resulting from gluon exchange.

The matrices $\lambda_{4}$ and $\lambda_{5}$ are two of the eight Gell-Mann matrices $\left(\lambda_{a=1 \ldots 8}\right.$, see Appendix B) which are the generators of these transformations. These $3 \times 3$ matrices are Hermitian and have zero trace.

If $\psi$ is a wavefunction representing a quark color state, then a mixed color state can be constructed from infinitesmal transformations like the following,

$$
\psi \rightarrow\left(I+i \sum_{a=1}^{8} \frac{\lambda_{a} \omega^{a}}{N}\right) \psi
$$

where $\omega^{a}$ are the parameters of the transformation, $N$ is a large integer, and $I$ is the identity matrix. We can then build up any finite rotation in color space by repeated application of this infinitesmal transformation.

$$
\psi \rightarrow \lim _{N \rightarrow \infty}\left(I+i \sum_{a=1}^{8} \frac{\lambda_{a} \omega^{a}}{N}\right)^{N} \psi=\exp \left(\sum_{a=1}^{8} i \lambda_{a} \omega^{a}\right) \psi
$$

This is analogous to a gauge transformation in the case,

$$
\psi \longrightarrow e^{i \theta} \psi
$$

where $\psi$ is an electromagnetic field. The exponential in eq. (6.23) represents a $3 \times 3$ matrix which is unitary (U) and has the special (S) property that it's determinant equals one. Hence, this theory is called an $\mathrm{SU}(3)$ theory. 


\section{E. The running coupling constant}

One of the important outcomes of quantum field theory is that the coupling strength $(g)$ is not constant but varies with the scale (energy,momemtum or distance range). This scale dependence of the coupling strength is related to the zeros of a certain function called the $\beta$-function,

$$
\beta(g)=\mu \frac{\partial g}{\partial \mu}=\frac{\partial g}{\partial \ln \mu}
$$

where $\mu$ is the energy or mass scale and where $\hbar=c=1$.

For Abelian quantum field theories such as quantum electrodynamics the $\beta$ function is positive and the coupling strength increases as the energy scale increases (or as the distance scale decreases). At low energy (long distances) the coupling or fine structure constant,

$$
\alpha=\frac{e^{2}}{4 \pi \epsilon_{o} \hbar c} \approx \frac{1}{137},
$$

increases to about $\frac{1}{127}$ at energies of order $90 \mathrm{GeV}$ or of the order of the $Z$ boson mass. In non-Abelian theories, this beta function is negative, and as a result, the coupling strength decreases at short distances $[23,24]$. This decrease is logarithmic,

$$
\alpha_{s}\left(\mu^{2}\right)=\frac{g^{2}}{4 \pi}=\frac{1}{\beta_{0}} \ln \left(\frac{\mu^{2}}{\lambda^{2}}\right)
$$

where $\lambda$ is the characteristc energy scale of $\mathrm{QCD}(\lambda \approx 200 \mathrm{MeV})$, and $\beta_{0}$ is a constant computed by Wilczek, Gross and Politzer. To compute the actual $\beta$-function for QCD, several Feynman diagrams are calculated to give the first quantum corrections to the wave function renormalization and to the three point gluon coupling. The final result is [83],

$$
\beta(g)=-\frac{g^{3}}{16 \pi^{2}}\left[\frac{11}{3} C_{2}-\frac{4}{3} T\right]+O\left(g^{5}\right)
$$

where $C_{2}$ and $T$ are called Casimir operators for the gluon and quark representations. $\beta(g)$ will be negative as long as $T$, which depends on the number of quarks, is not too large. This negative $\beta(g)$ means that $\partial g / \partial \mu$ is also negative and the coupling $g$ decreases with increasing $\mu$. This means as quarks come closer together their force of attraction decreases eventually to zero, a property called asymptotic freedom. 


\section{F. Flavor/isospin structure}

The flavor/isospin structure of $\mathcal{O}_{h}$ and $\mathcal{O}_{\pi a_{1}}$ needs to be determined. For $\mathcal{O}_{h}$ we choose

$$
\bar{\psi} \psi=\bar{d} u \sim\left|\frac{1}{2} \frac{1}{2}\right\rangle\left|\frac{1}{2} \frac{1}{2}\right\rangle,
$$

where the "ket" states represent isospin $I=\frac{1}{2}$ and $I_{3}=+\frac{1}{2}$. The direct product state has total isospin $I=1$ and $I_{3}=+1$.

Because the strong interactions conserve isospin (assumimg $m_{u}=m_{d}$ ), the $\pi a_{1}$ operator $\mathcal{O}_{\pi a_{1}}$ should also have the same total isospin quantum numbers in order to couple maximally to $\mathcal{O}_{h}$. The corresponding Clesch-Gordon decomposition is

$$
\begin{aligned}
|11\rangle_{\pi a_{1}}= & \frac{1}{\sqrt{2}}\left(|11\rangle_{\pi}|10\rangle_{a_{1}}-|10\rangle_{\pi}|11\rangle_{a_{1}}\right) . \\
& =\frac{1}{\sqrt{2}}\left(\pi^{+} a_{1}^{0}-\pi^{0} a_{1}^{+}\right)
\end{aligned}
$$

where the one-meson states have the flavor assignment

$$
|11\rangle=\left|\frac{1}{2} \frac{1}{2}\right\rangle\left|\frac{1}{2} \frac{1}{2}\right\rangle=\bar{d} u \quad, \quad \pi^{+} \quad \text { or } \quad a_{1}^{+}
$$

and,

$$
\begin{gathered}
|10\rangle=\frac{1}{\sqrt{2}}\left(\left|\frac{1}{2} \frac{1}{2}\right\rangle\left|\frac{1}{2} \frac{-1}{2}\right\rangle+\left|\frac{1}{2} \frac{-1}{2}\right\rangle\left|\frac{1}{2} \frac{1}{2}\right\rangle\right) \\
=\frac{1}{\sqrt{2}}(\bar{d} d+\bar{u} u), \pi^{0} \text { or } a_{1}^{0} .
\end{gathered}
$$

So the combined $\pi a_{1}$ system is

$$
|11\rangle_{\pi a_{1}}=\frac{1}{\sqrt{2}}\left((\bar{d} u)_{\pi} \frac{1}{\sqrt{2}}(\bar{d} d+\bar{u} u)_{a_{1}}-\frac{1}{\sqrt{2}}(\bar{d} d+\bar{u} u)_{\pi}(\bar{d} u)_{a_{1}}\right) .
$$

The hybrid decay written in terms of charged particles is therefore,

$$
h^{+} \longrightarrow \frac{1}{2}\left(\pi^{+} a_{1}^{0}-\pi^{0} a_{1}^{+}\right) .
$$




\section{G. Maximum entropy method analysis}

An alternative to using effective mass functions is the application of a maximum entropy method(MEM) or a constrained form of Bayesian inference [84]. In this method, a data set $D$, say eigenvalues of a correlation matrix $\mathrm{C}$ or $\Lambda$ is associated with a certain parameter set $\rho$ with a probability $P[\rho \leftarrow D]$. For analyzing mass spectra, $C$ is the set of correlator values and the parameter set $\rho$ represents a spectral density function. A correlator $\operatorname{model}(F)$ is then written,

$$
F\left(\rho \mid t, t_{0}\right)=\int d \omega \rho(\omega) e^{-\omega\left(t-t_{0}\right)}
$$

This is similar to a sum of decaying exponentials, see eq. (2.37). After a set of $\rho$ values is selected, the correlator model $(F)$ is computed and compared to the actual correlator values $(C)$. A probability $P[\rho \leftarrow C]$ is constructed from the $\chi^{2}$-distance between $F$ and $C$. This probability also gets a contribution from an entropy function which is defined as,

$$
S=-\int d \omega \rho(\omega) \ln [\rho(\omega)]
$$

The idea is to minimize the $\chi^{2}$-distance while maximizing the entropy. The spectral density function is actually a discrete sum of $\delta$-peaks,

$$
\rho(\omega)=\sum_{n \neq 0} \delta\left(\omega-\omega_{0}\right)|<n| \Phi|0>|^{2}
$$

that have finite widths. Physical information is contained in the low $w$-moments of each peak. For example, the quantity $|<n| \Phi|0>|^{2}$ is related to the volume of the peak, and the peak energy $E_{n}$ is the mean value of $w$.

In Fig. 18, results of a MEM analysis are displayed for two eigenvalues of the correlation matrix used in this project. The spectral density has peaks at $\sim 1.0$ $\mathrm{GeV}$ and $\sim 2.0 \mathrm{GeV}$ for these eigenvalues. If there are multiple peaks, then only the lowest energy or ground state peaks are taken. 

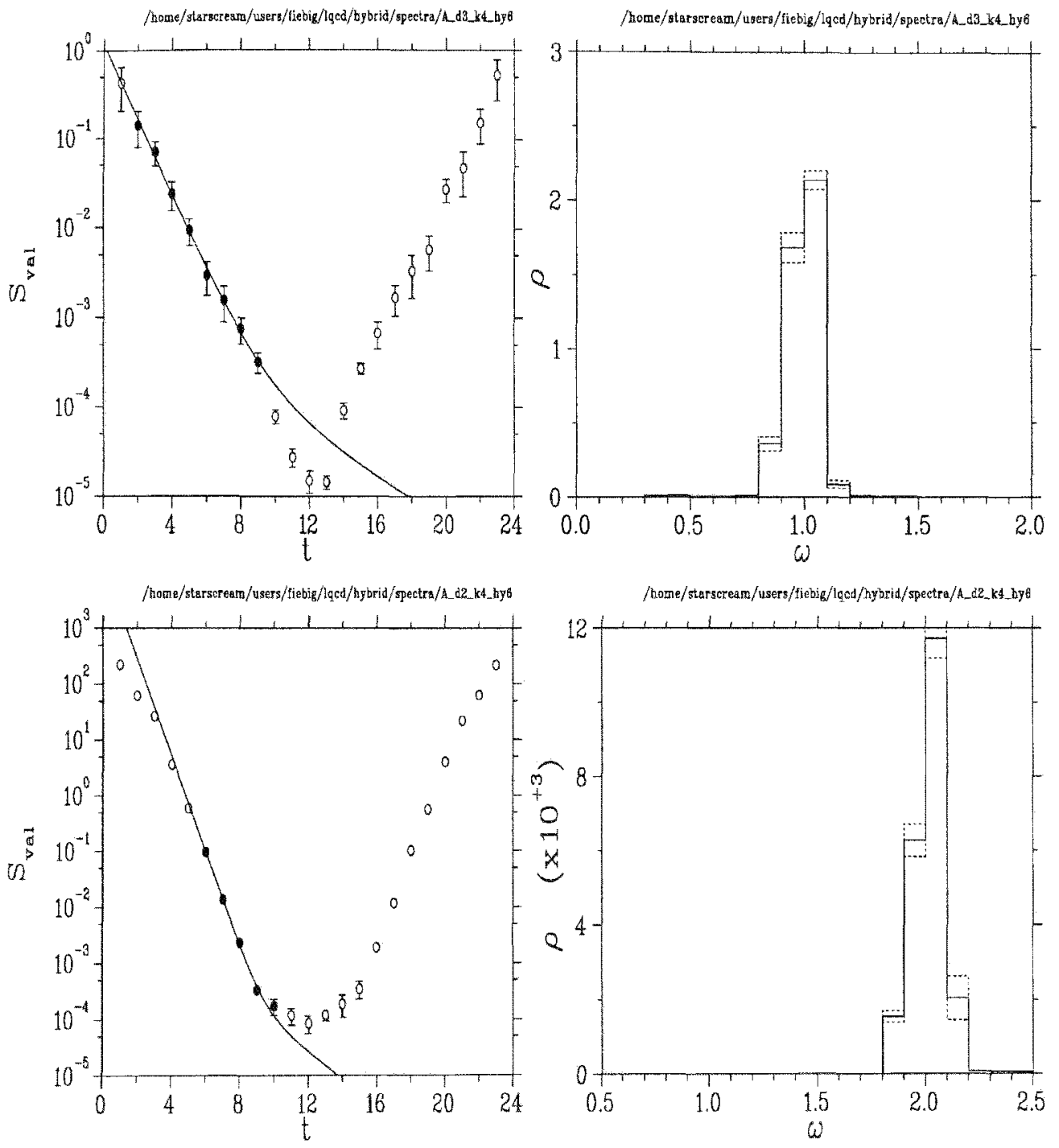

Figure 18: Fits to the correlation functions and the resulting spectral densities using the maximum entropy method for eigenvalues $\lambda_{3}$ (upper panels) and $\lambda_{2}$ (lower panels). The lattice size is $12^{3} \times 24$ and kappa $=.1400$. Unfilled data points are not used in the fit. 
H. Collection of graphs

Here we collect together various graphs, many of which are not shown in the seven chapters of this dissertation.
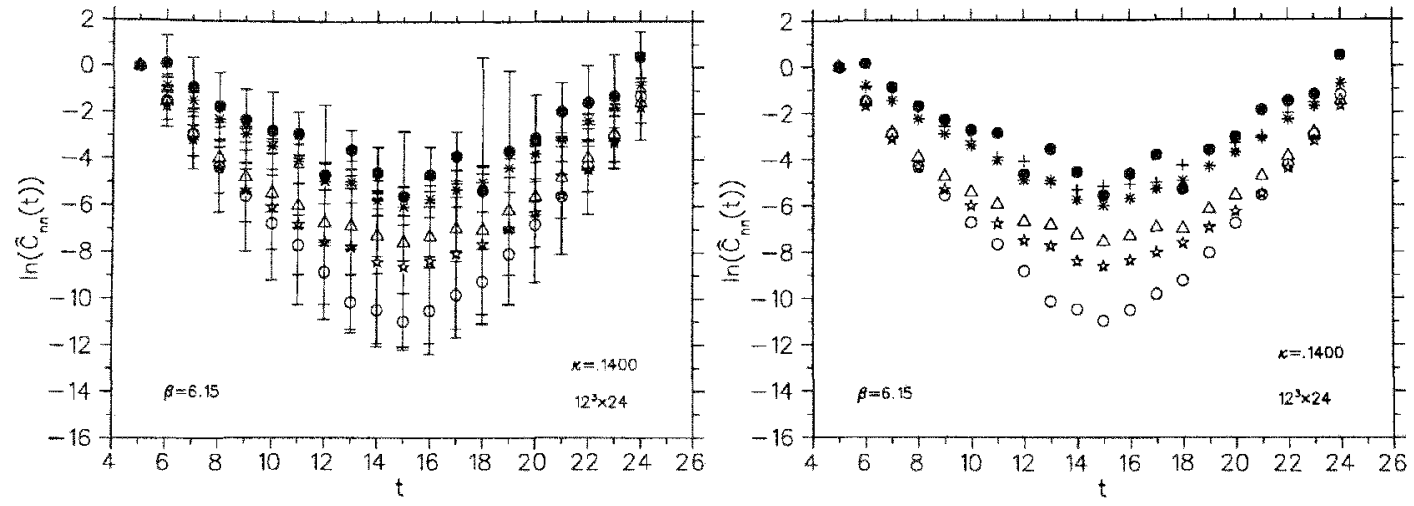

Figure 19: Eigenvalues of the $6 \times 6$ correlation matrix for the $12^{3} \times 24$ lattice. Diagonalized and normalized at $\mathrm{t}=5$. Projected back to $\mathrm{t}=5$.
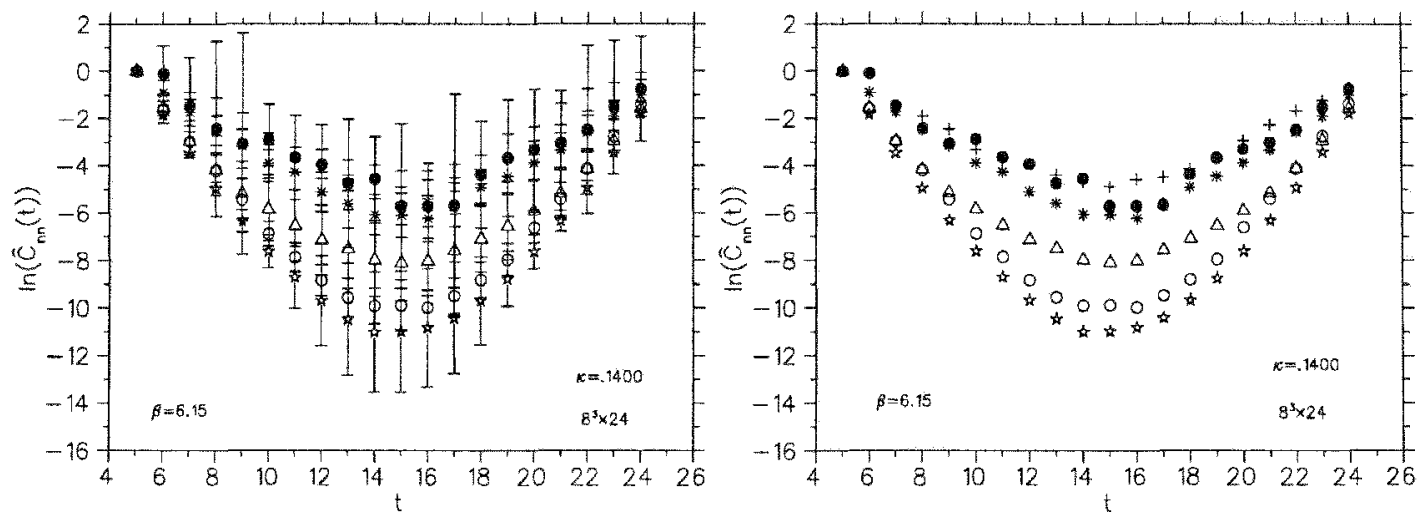

Figure 20: Eigenvalues of the $6 \times 6$ correlation matrix for the $8^{3} \times 24$ lattice. Diagonalized and normalized at $t=5$. Projected back to $t=5$. 

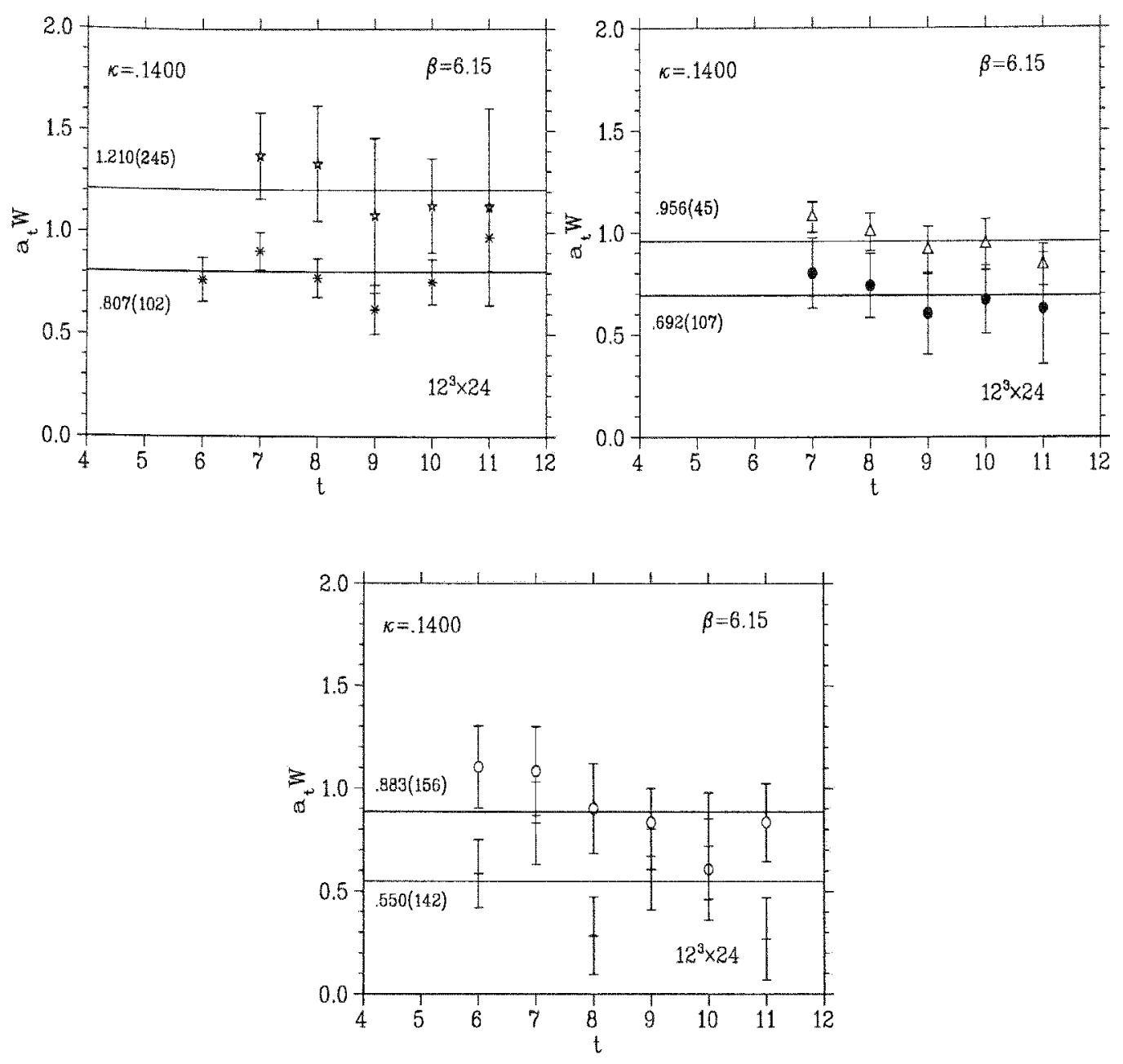

Figure 21: Effective masses for all six eigenvalues of the correlation matrix on the $12^{3} \times 24$ lattice at the lightest pion mass. 

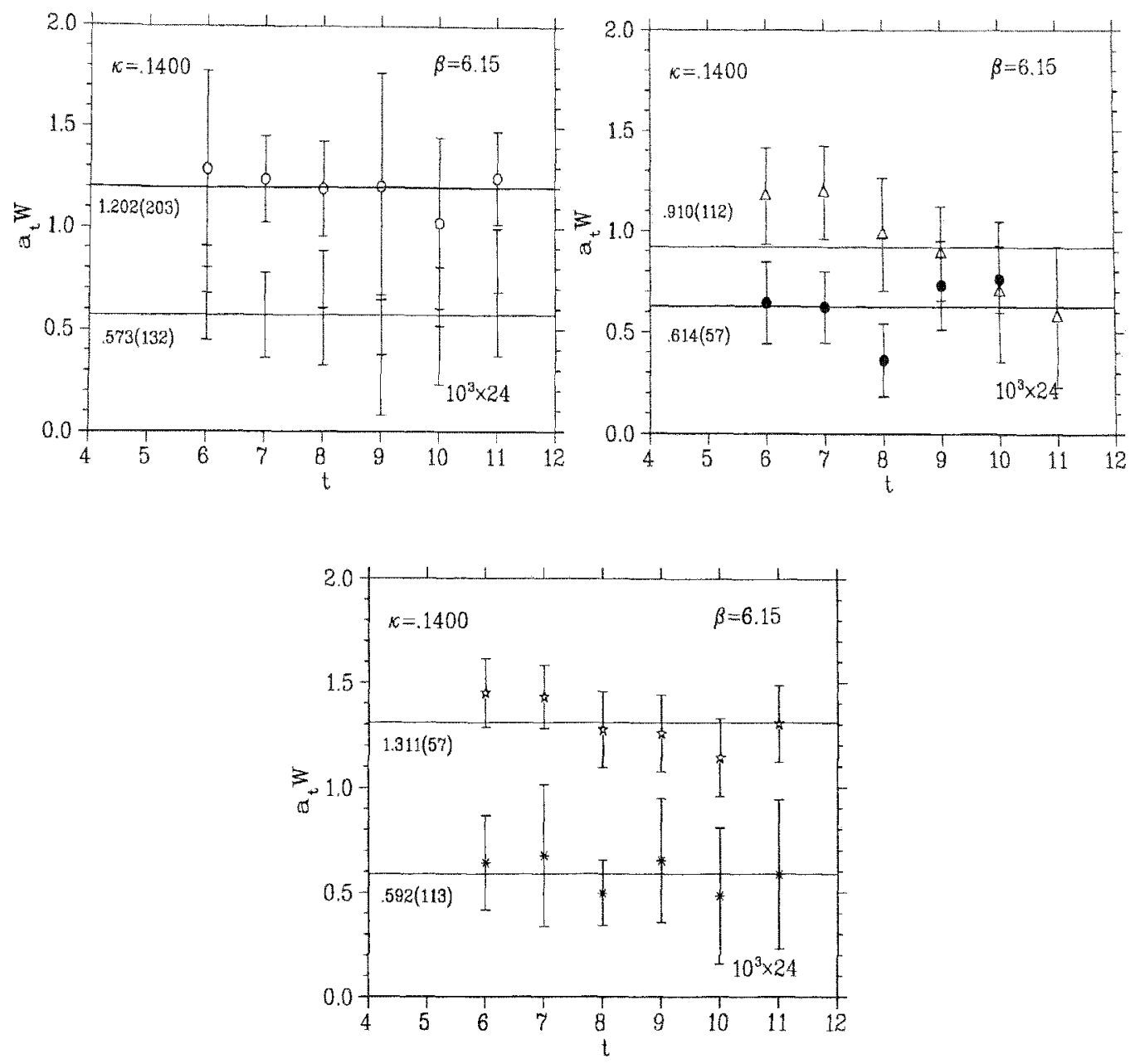

Figure 22: Effective masses for all six eigenvalues of the correlation matrix on the $10^{3} \times 24$ lattice at the lightest pion mass. 


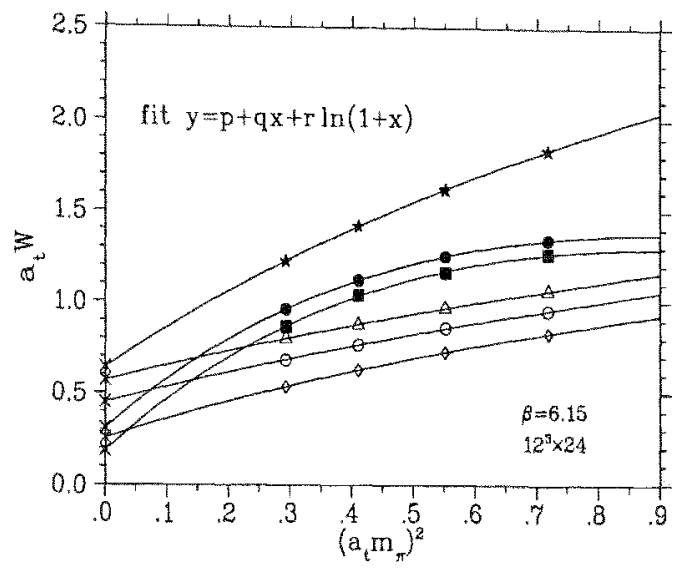

Figure 23: Energy spectrum for the $12^{3} \times 24$ lattice extrapolated to zero pion mass. Error bars omitted for clarity.

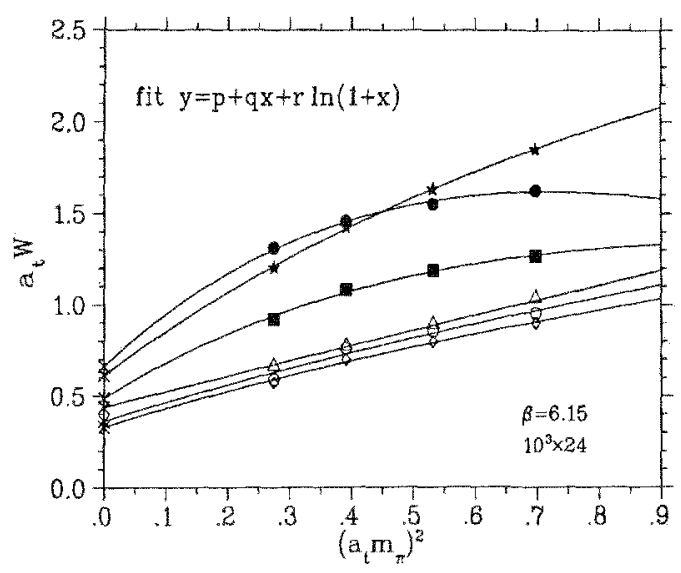

Figure 24: Energy spectrum for the $10^{3} \times 24$ lattice extrapolated to zero pion mass. Error bars omitted for clarity. 


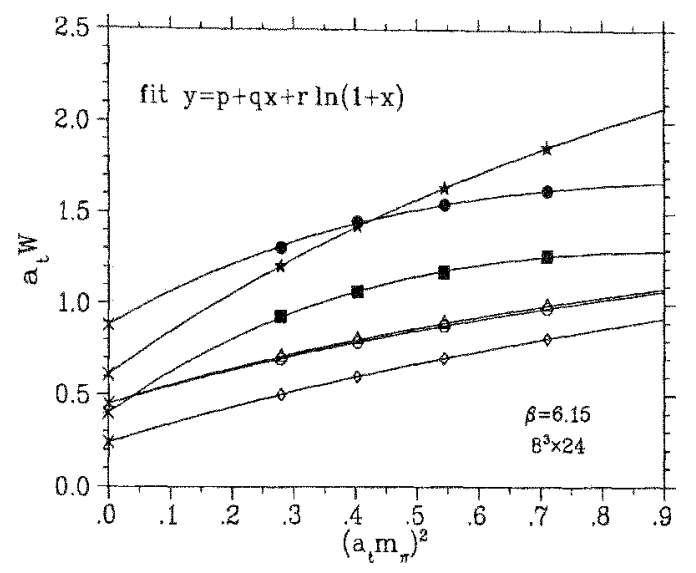

Figure 25: Energy spectrum for the $8^{3} \times 24$ lattice extrapolated to zero pion mass. Error bars omitted for clarity.
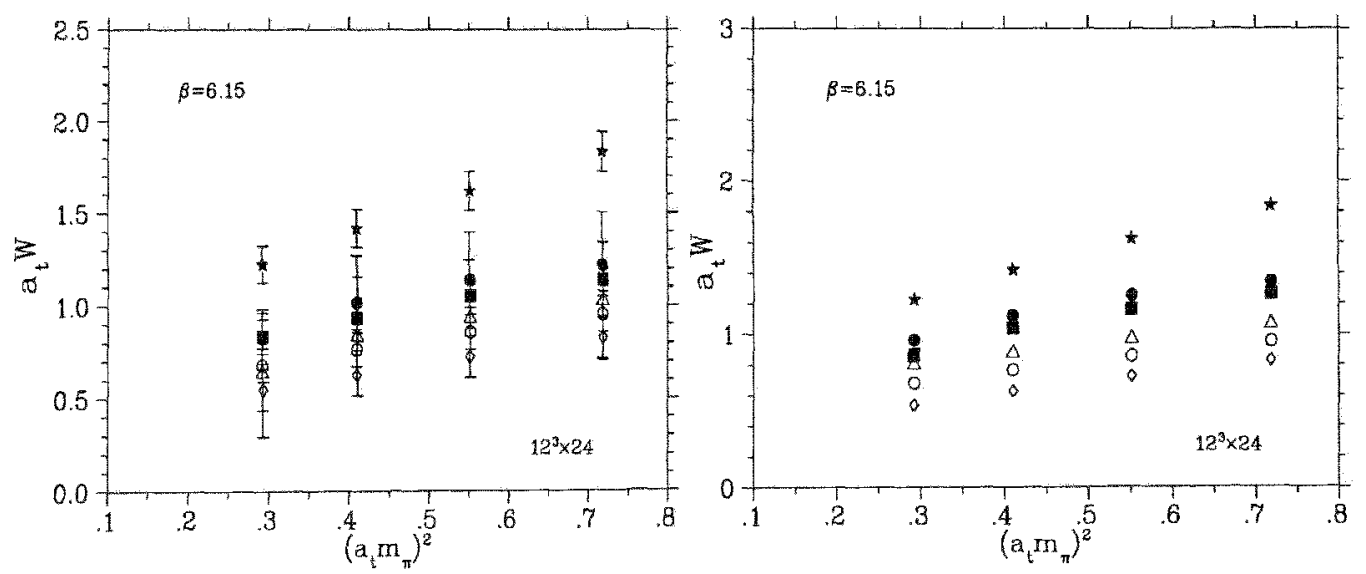

Figure 26: Energy spectrum for the $12^{3} \times 24$ lattice. Shown with and without error bars. 

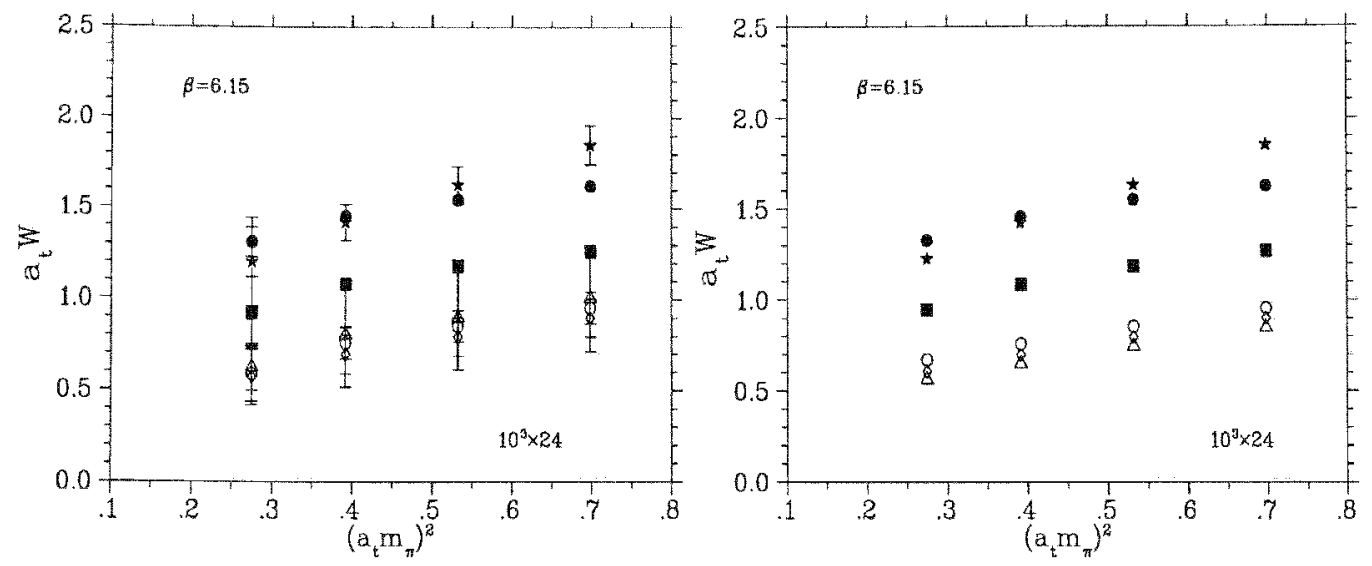

Figure 27: Energy spectrum for the $10^{3} \times 24$ lattice. Shown with and without error bars.
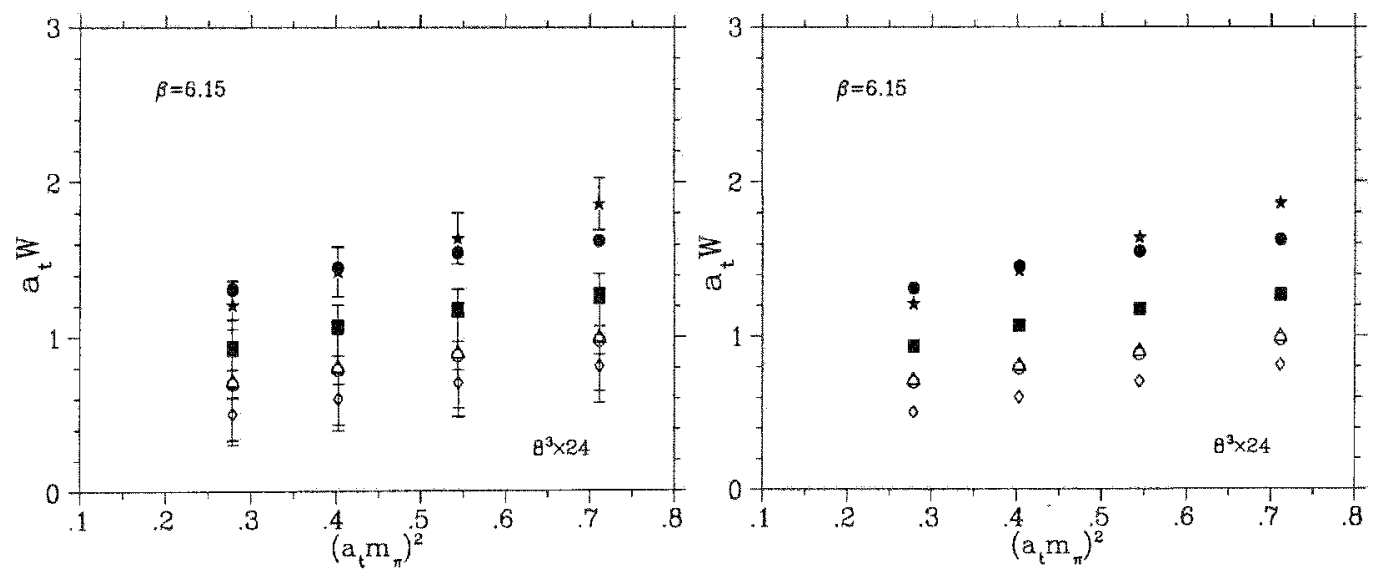

Figure 28: Energy spectrum for the $8^{3} \times 24$ lattice. Shown with and without error bars. 


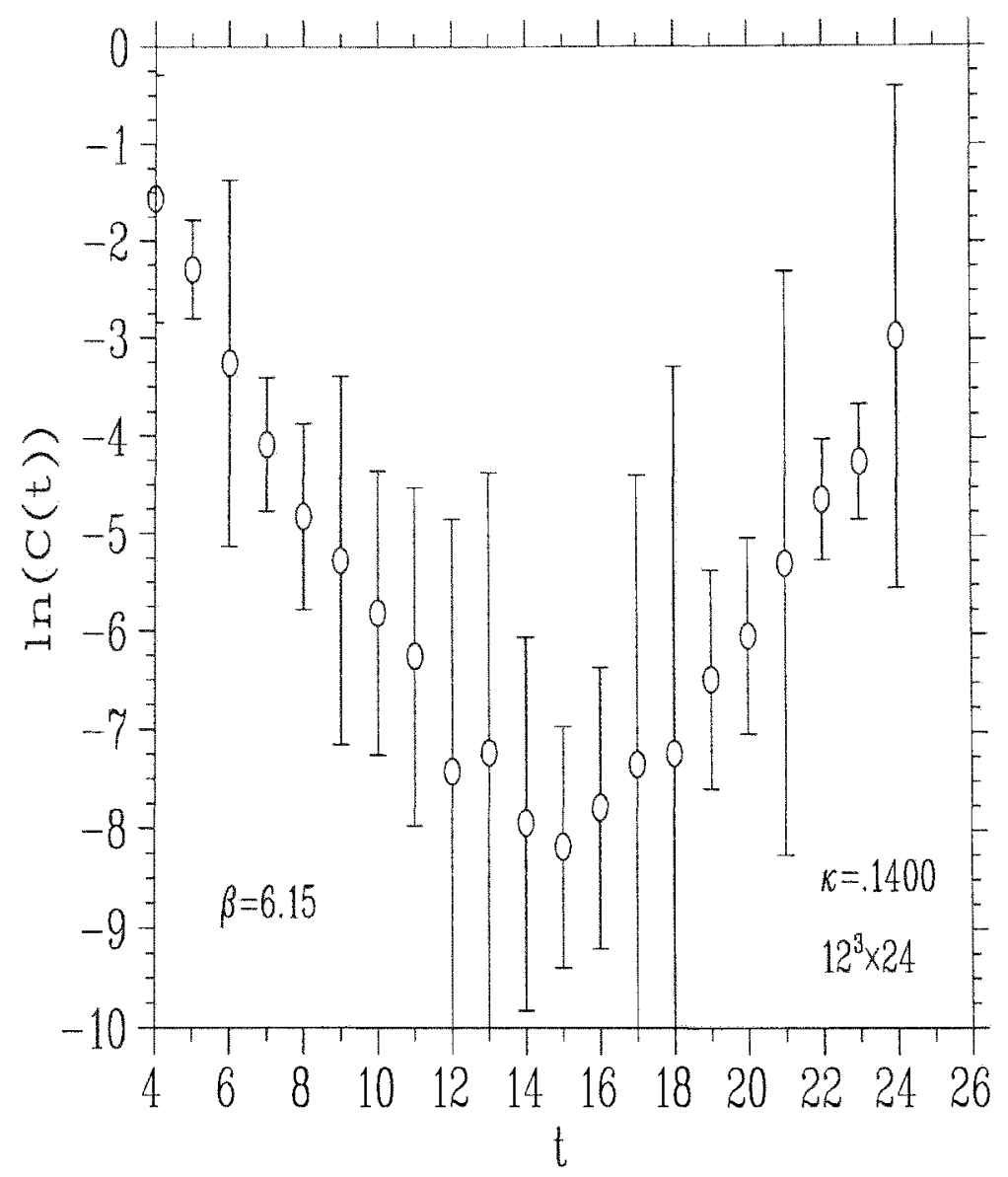

Figure 29: Exotic hybrid correlation function for 2 smearings at source and sink points on the $12^{3} \times 24$ lattice. 


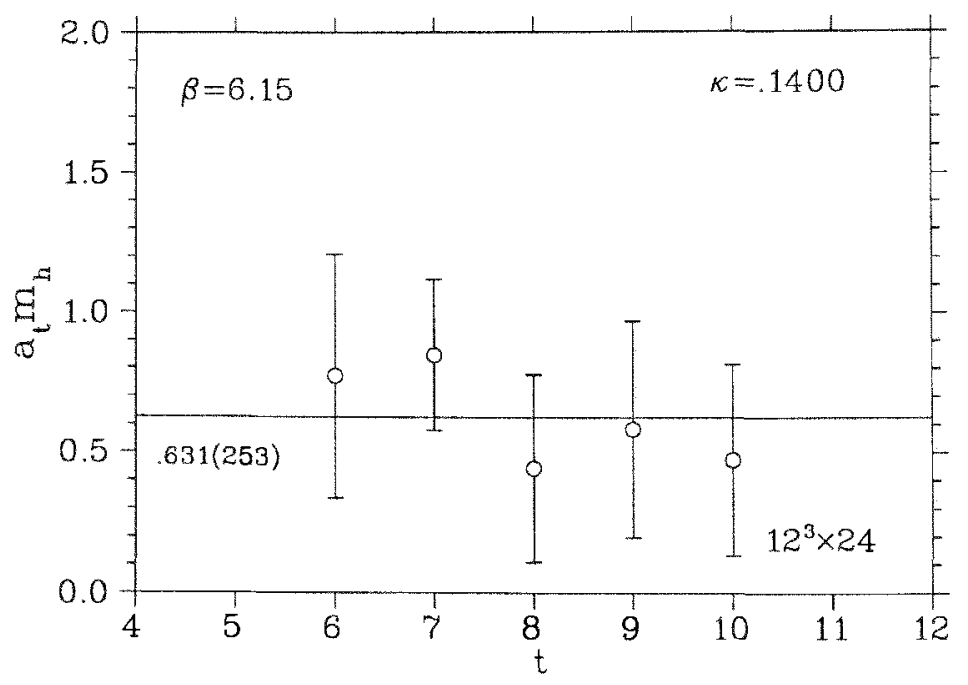

Figure 30: Effective mass of the exotic hybrid on the $12^{3} \times 24$ lattice for $\kappa=.1400$.

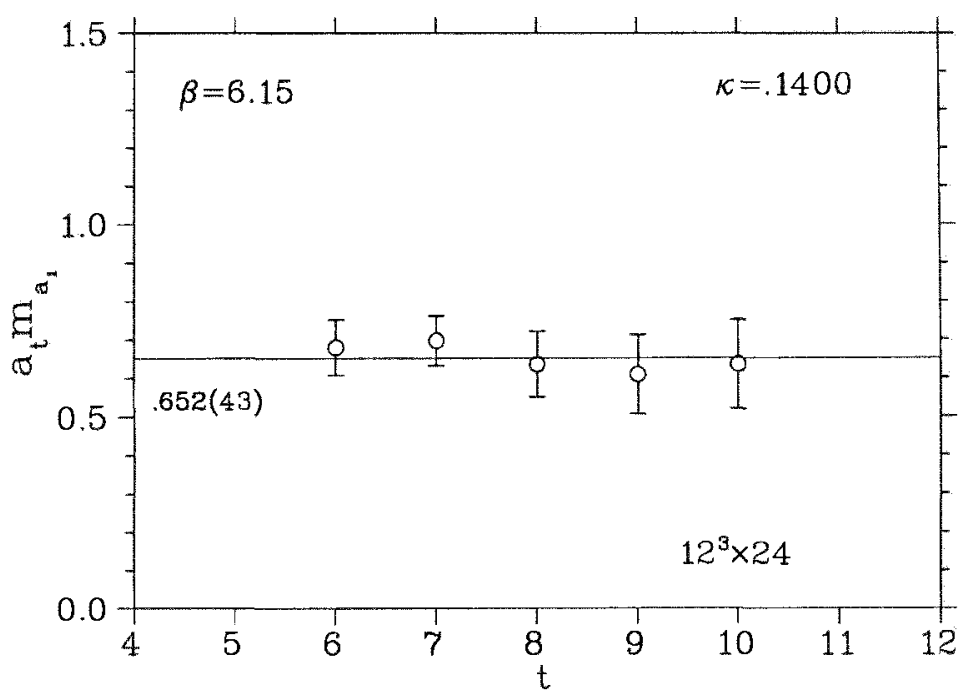

Figure 31: An effective mass of the a1 meson on the $12^{3} \times 24$ lattice at $\kappa=.1400$. 


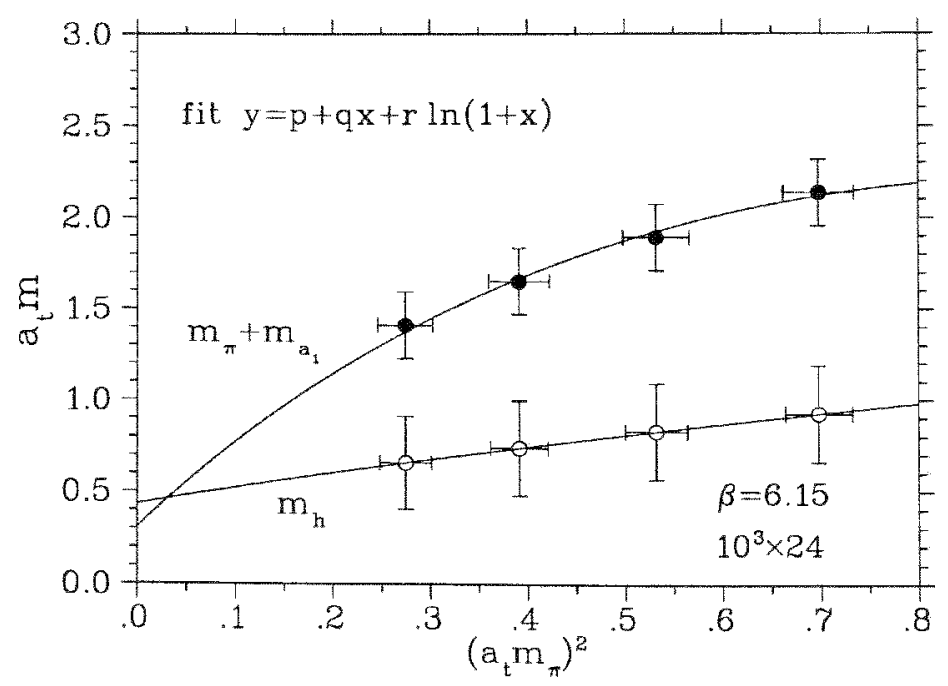

Figure 32: Effective masses for the hybrid meson(unfilled dots) and $a_{1}+\pi$ two meson system(filled dots) on the $10^{3} \times 24$ lattice( 2 smearings at source and sink).

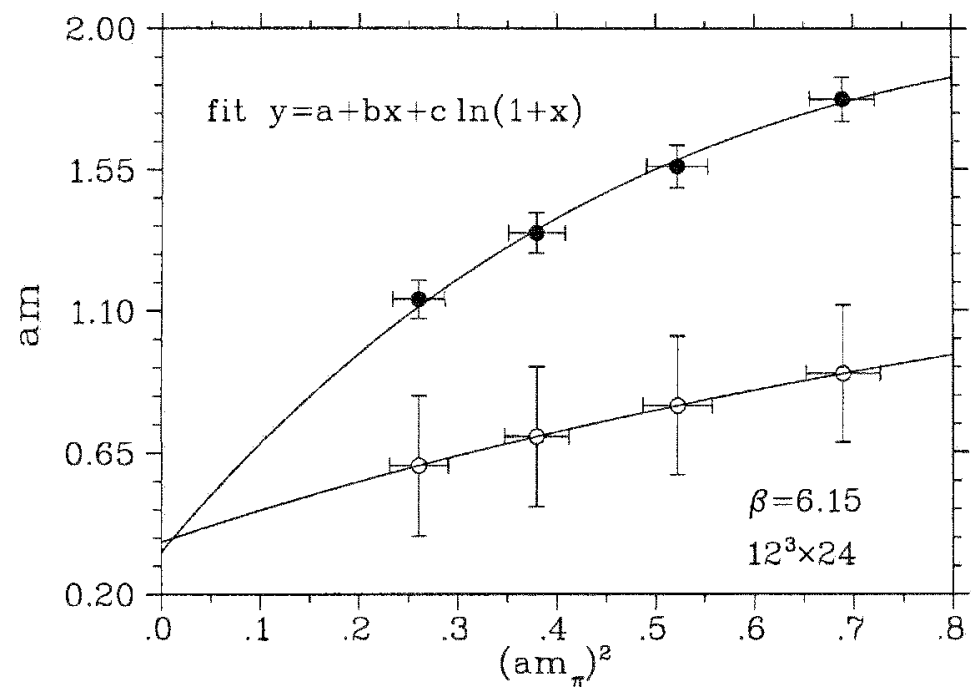

Figure 33: Effective masses for the hybrid meson(unfilled dots) and $a_{1}+\pi$ two meson system(filled dots) on the $12^{3} \times 24$ lattice( 2 smearings at source and sink). 

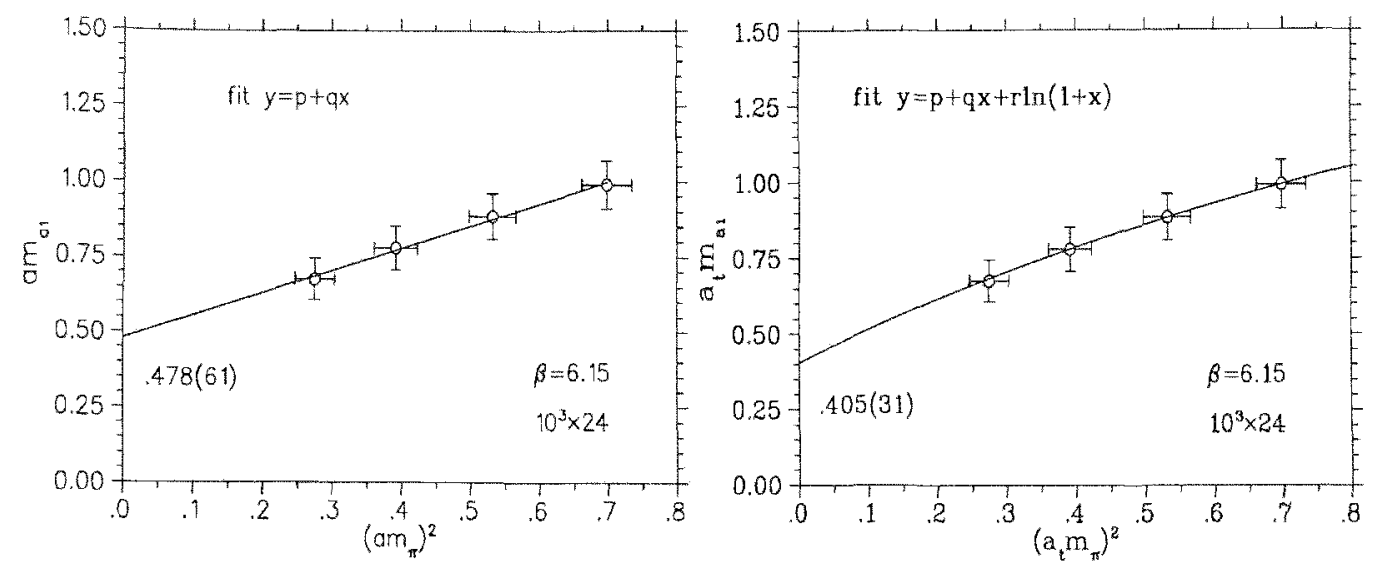

Figure 34: Effective masses of the a1 meson with linear and logarithmic fits on the $10^{3} \times 24$ lattice.

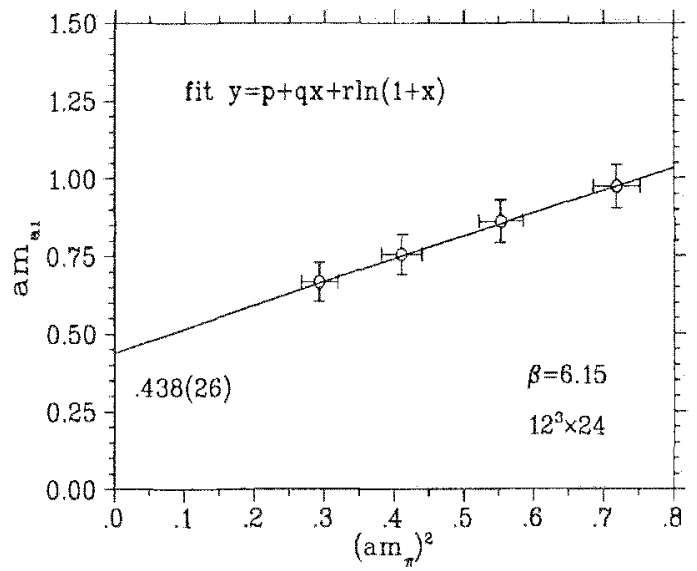

Figure 35: Effective masses of the a1 meson on the $12^{3} \times 24$ lattice . 


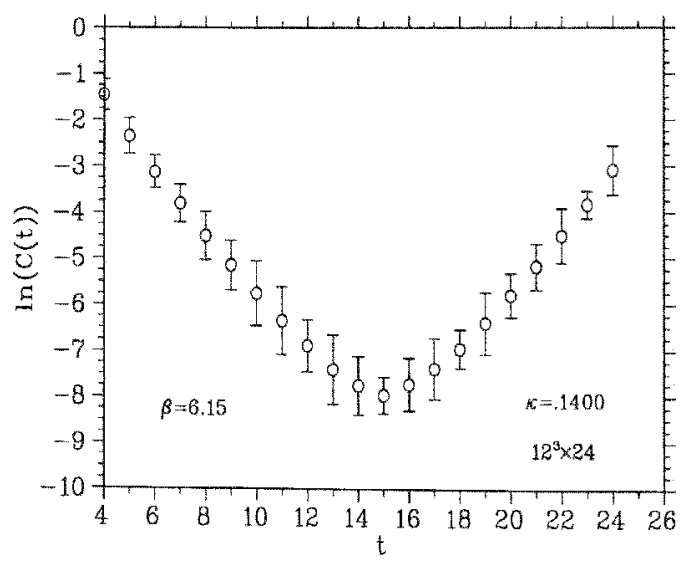

Figure 36: Correlation function for the a1 meson with 2 smearings at source and sink points on the $12^{3} \times 24$ lattice.
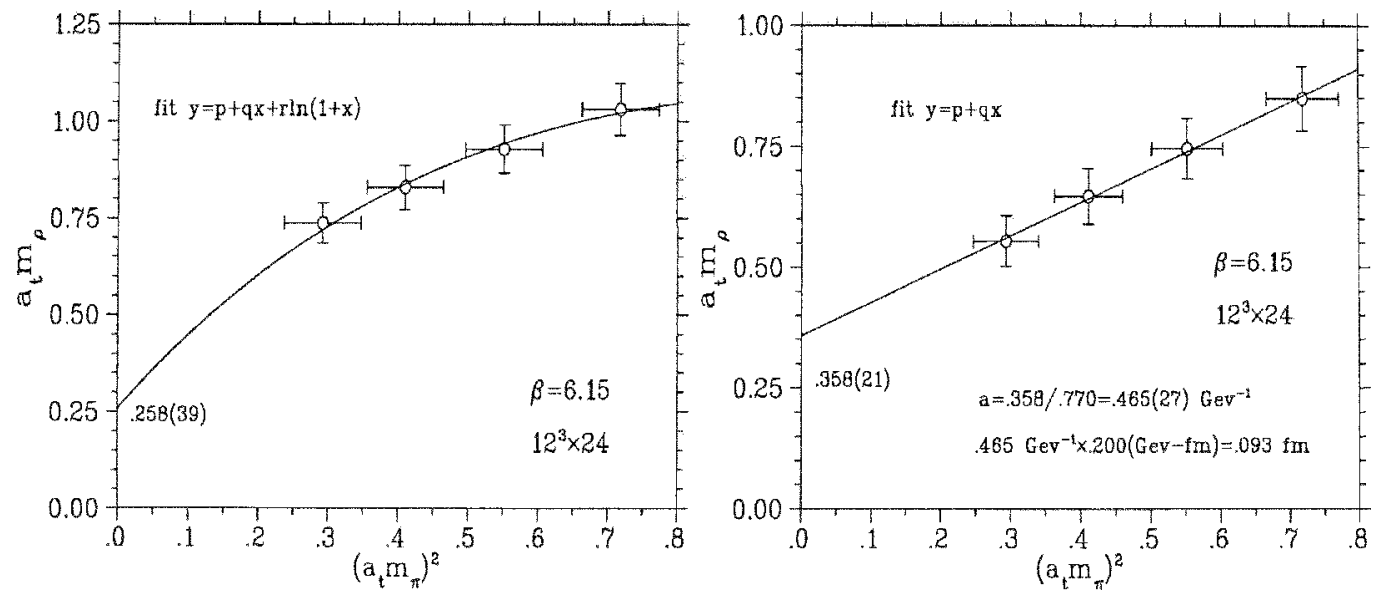

Figure 37: Lattice constants for the $12^{3} \times 24$ lattice using linear and logarithmic fits. 

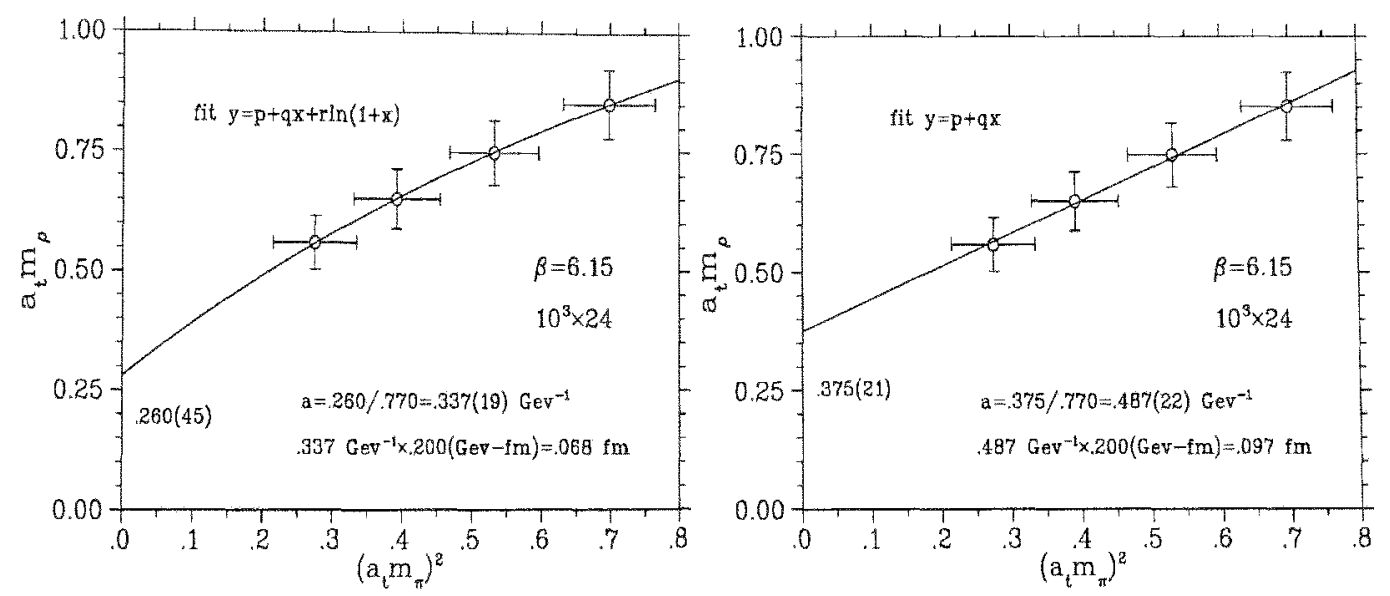

Figure 38: Lattice constants for the $10^{3} \times 24$ lattice using linear and logarithmic fits. 
August 18,1955

1975

1983

1984-1988

1988-1993

1994-1997

$1997-2000$

2001-2006
Born, Pittsburgh,PA.

B.S. Mechanical Enginnering Clemson University Clemson S.C.

M.S. Mechanical Engineering State University of New York Buffalo, New York

Research Engineer

Cincinnati Milacron

Cincinnati, Ohio

Lead Engineer

Spectra-Physics

Dayton, Ohio

Project Manager

Coburn Optical Industries Muskogee, OK.

Manufacturing Engineer

Cordis Corporation

Miami, Florida

Research Assistant

Florida International University Miami, Florida 


\section{PUBLICATIONS AND PRESENTATIONS}

Cook, M. S. and Fiebig, H. R.(2002). A Lattice Study of Interaction Mechanisms in a Heavy Light,Meson-Meson System. [hep-lat/0210054].

Cook, M. S.(2004). Exotic Meson Decay Widths using Lattice QCD. Presentation at Hampton University Graduate School held at Jefferson National Laboratory, July 2004, Newport News, Virginia.

Cook, M. S. and Fiebig, H. R.(2005). Hybrid Exotic Meson Decay Width. In proceedings of The 23rd International Symposium on Lattice Field Theory, 25-30 July 2005, Trinity College, Dublin, Ireland, PoS(LAT2005)062, [hep-lat/0509028].

Cook, M. S. and Fiebig, H. R.(2005). Interaction Studies of a heavy-light mesonbaryon system. In proceedings of The 23rd International Symposium on Lattice Field Theory, 25-30 July 2005, Trinity College, Dublin, Ireland, PoS(LAT2005)087, [hep-lat/0509025]. 